\title{
Equivariant CW-complexes and the orbit category
}

\author{
Ian Hambleton, Semra Pamuk and Ergün Yalçın*
}

\begin{abstract}
We give a general framework for studying $G$-CW complexes via the orbit category. As an application we show that the symmetric group $G=S_{5}$ admits a finite $G$-CW complex $X$ homotopy equivalent to a sphere, with cyclic isotropy subgroups.
\end{abstract}

Mathematics Subject Classification (2010). 57S17, 55U15, 20J05, 18Gxx.

Keywords. Finite group actions on spheres, orbit category, chain complexes.

\section{Introduction}

A good algebraic setting for studying actions of a group $G$ with isotropy in a given family of subgroups $\mathcal{F}$ is provided by the category of $R$-modules over the orbit category $\Gamma_{G}=\operatorname{Or}_{\mathcal{F}} G$, where $R$ is a commutative ring with unit. This theory was established by Bredon [6], tom Dieck [10] and Lück [20], and further developed by many authors (see, for example, Jackowski-McClure-Oliver [17], §5, Brady-LearyNucinkis [5], Symonds [34], [35], Grodal [14], Grodal-Smith [15]). In particular, the category of $R \Gamma_{G}$-modules is an abelian category with Hom and tensor product, and has enough projectives for standard homological algebra.

In this paper, we will study finite group actions on spheres with non-trivial isotropy, generalizing the approach of Swan [32] to the spherical space form problem through periodic projective resolutions. A finite group is said to have rank $k$ if $k$ is the largest integer such that $G$ has an elementary abelian subgroup $C_{p} \times \cdots \times C_{p}$ of rank $k$ for some prime $p$. A rank 1 group $G$ has periodic cohomology, and Swan showed that this was a necessary and sufficient condition for the existence of a finite free $G-\mathrm{CW}$ complex $X$, homotopy equivalent to a sphere.

The work of Adem-Smith [1] concerning free actions on products of spheres led to the following open problem:

Question. If $G$ is a rank 2 finite group, does there exist a finite $G$-CW complex $X \simeq S^{n}$ with rank 1 isotropy ?

\footnotetext{
${ }^{*}$ Research partially supported by NSERC Discovery Grant A4000. The third author is partially supported by TÜBITAK-BDP and TÜBA-GEBIP/2005-16.
} 
If $G$ is a finite $p$-group of rank 2, then there exist orthogonal linear representations $V$ so that $S(V)$ has rank 1 isotropy (see [12]). If $G$ is not of prime power order, representation spheres with rank 1 isotropy do not exist in general: a necessary condition is that $G$ has a $p$-effective character for each prime $p$ dividing $|G|$ (see Theorem 47 in [18]). In Proposition 48 of [18] it is claimed that this condition is also sufficient for an affirmative answer to the $G-\mathrm{CW}$ question above, but the discussion on p. 831 of [18] does not provide a construction for $X$.

Our main result concerns the first non-trivial case: the permutation group $G=S_{5}$ of order 120, which has rank 2 but no linear action with rank 1 isotropy on any sphere, although it does admit $p$-effective characters for $p=2,3,5$.

Theorem A. The permutation group $G=S_{5}$ admits a finite $G$-CW complex $X \simeq S^{n}$, such that $X^{H} \neq \emptyset$ implies that $H$ is a rank 1 subgroup of 2-power order.

Remark 1.1. It is an interesting problem for future work to decide if the group $G=S_{5}$ can act smoothly on $S^{n}$ with rank 1 isotropy.

In order to prove this result we develop further techniques over the orbit category, which may have some independent interest. A well-known theorem of Rim [29] shows that a module $M$ over the group ring $\mathbb{Z} G$ is projective if and only if its restriction $\operatorname{Res}_{P}^{G} M$ to any $p$-Sylow subgroup is projective. Over the orbit category we have a similar statement localized at $p$ (see Theorem 3.9).

Theorem B. Let $G$ be a finite group and let $R=\mathbb{Z}_{(p)}$. Then an $R \Gamma_{G}$-module $M$ has a finite projective resolution with respect to a family of p-subgroups if and only if its restriction $\operatorname{Res}_{P}^{G} M$ has a finite projective resolution over any $p$-Sylow subgroup $P \leq G$.

Remark 1.2. For modules over the group ring $R G$, those having finite projective resolutions are already projective. Over the orbit category, these two properties are distinct.

Another useful feature of homological algebra over group rings is the detection of group cohomology by restriction to the $p$-Sylow subgroups. Here is an important concept in group cohomology (see for example [33]).

Definition 1.3. For a given prime $p$, we say that a subgroup $H \subseteq G$ controls $p$-fusion provided that

(i) $p \nmid|G / H|$, and

(ii) whenever $Q \subseteq H$ is a $p$-subgroup, and there exists $g \in G$ such that $Q^{g}:=$ $g^{-1} Q g \subseteq H$, then $g=c h$ where $c \in C_{G}(Q)$ and $h \in H$. 
One reason for the importance of this definition is the fact that the restriction map

$$
H^{*}\left(G ; \mathbb{F}_{p}\right) \rightarrow H^{*}\left(H ; \mathbb{F}_{p}\right)
$$

is an isomorphism if and only if $H$ controls $p$-fusion in $G$ (see [25], [33]). We have the following generalization (see Theorem 5.1) for functors of cohomological type over the orbit category (with respect to any family $\mathcal{F}^{\text {) }}$.

Theorem C. Let $G$ be a finite group, $R=\mathbb{Z}_{(p)}$, and $H \leq G$ a subgroup which controls p-fusion in G. If $M$ is an $R \Gamma_{G}$-module and $N$ is a cohomological Mackey functor, then the restriction map

$$
\operatorname{Res}_{H}^{G}: \operatorname{Ext}_{R \Gamma_{G}}^{n}(M, N) \rightarrow \operatorname{Ext}_{R \Gamma_{H}}^{n}\left(\operatorname{Res}_{H}^{G} M, \operatorname{Res}_{H}^{G} N\right)
$$

is an isomorphism for $n \geqslant 0$, provided that the centralizer $C_{G}(Q)$ of any p-subgroup $Q \leq H$, with $Q \in \mathscr{F}$, acts trivially on $M(Q)$ and $N(Q)$.

The construction of the $G$-CW complex $X$ for $G=S_{5}$ and the proof of Theorem A is carried out in Section 9. We first construct finite projective chain complexes $\mathbf{C}^{(p)}$ over the orbit categories $R \Gamma_{G}$, with $R=\mathbb{Z}_{(p)}$, separately for the prime $p=2,3,5$ dividing $|G|$. In each case, the isotropy family $\mathcal{F}$ consists of the rank 1 subgroups of 2-power order in $G$.

The chain complexes $\mathbf{C}^{(p)}$ all have the same dimension function (see Definition 8.2). We prescribe a non-negative function $\underline{n}: \mathcal{F} \rightarrow \mathbb{Z}$, with the property that $\underline{n}(K) \leq \underline{n}(H)$ whenever $H$ is conjugate to a subgroup of $K$. Then, by construction, each complex $\mathbf{C}^{(p)}$ has the $R$-homology of an $\underline{n}$-sphere: for each $K \in \mathcal{F}$, the complexes $\mathbf{C}^{(p)}(K)$ have homology $H_{i}=R$ only in two dimensions $i=0$ and $i=\underline{n}(K)$. In other words, the complexes $\mathbf{C}^{(p)}$ are algebraic versions of tom Dieck's homotopy representations ([10], II.10).

In the case $p=2$, we start with the group $H=S_{4}$ acting by orthogonal rotations on the 2-sphere. A regular $H$-equivariant triangulation of an inscribed cube or octahedron gives a finite projective chain complex over $R \Gamma_{H}$. Then we use Proposition 6.4, a chain complex version of Theorem $\mathrm{C}$, to lift it to a finite projective complex over $R \Gamma_{G}$. For $p=3$ and $p=5$, the $p$-rank of $S_{5}$ is 1 , and there exists a periodic complex over the group ring $R G$ (see Swan [32], Theorem B). We start with a periodic complex over $R G$ and add chain complexes to this complex, for every nontrivial subgroup $K \in \mathcal{F}$, to obtain the required complex $\mathbf{C}^{(p)}$ over $R \Gamma_{G}$.

We use the theory of algebraic Postnikov sections by Dold [11] to glue the complexes together to form a finite projective $\mathbb{Z} \Gamma_{G}$ chain complex (see Section 6). We complete the chain complex construction by varying the finiteness obstruction to obtain a complex of free $\mathbb{Z} \Gamma_{G}$-modules, and then we prove a realization theorem (see Section 8) to construct the required $G$-CW complex $X \simeq S^{n}$.

Throughout the paper, a family of subgroups will always mean a collection of subgroups which is closed under conjugation and taking subgroups. Also, unless otherwise stated, all modules are finitely generated. 
Acknowledgement. The authors would like to thank the referee for many valuable criticisms and suggestions. The third author would also like to thank McMaster University for the support provided by an H. L. Hooker Visiting Fellowship, and the Department of Mathematics \& Statistics at McMaster for its hospitality while this paper was written.

\section{Modules over small categories}

Our main source for the material in this section is Lück [20], §9, §17 (see also §I.10, $\S I .11$ in [10]). We include it here for the convenience of the reader.

Let $R$ be a commutative ring. We denote the category of $R$-modules by $R$-Mod. For a small category $\Gamma$ (i.e., the objects $\operatorname{Ob}(\Gamma)$ of $\Gamma$ form a set), the category of right $R \Gamma$-modules is defined as the category of contravariant functors $\Gamma \rightarrow R$-Mod, where the objects are functors $M(-): \Gamma \rightarrow R$-Mod and morphisms are natural transformations. Similarly, we define the category of left $R \Gamma$-modules as the category of covariant functors $N(-): \Gamma \rightarrow R$-Mod. We denote the category of right $R \Gamma$ modules by Mod- $R \Gamma$ and the category of left $R \Gamma$-modules by $R \Gamma$-Mod.

The category of covariant or contravariant functors from a small category to an abelian category has the structure of abelian category which is object-wise induced from the abelian category structure on abelian groups (see [23], Chapter 9, Proposition 3.1). Hence the category of $R \Gamma$-modules is an abelian category where the notions submodule, quotient module, kernel, image, and cokernel are defined object-wise. The direct sum of $R \Gamma$-modules is given by taking the usual direct sum object-wise.

Example 2.1. The most important example for our applications is the orbit category of a finite group. Let $G$ be a finite group and let $\mathcal{F}$ be a family of subgroups of $G$ which is closed under conjugation and taking subgroups. The orbit category $\operatorname{Or}(G)$ is the category whose objects are subgroups $H$ of $G$ or coset spaces $G / H$ of $G$, and the morphisms $\operatorname{Mor}(G / H, G / K)$ are given by the set of $G$-maps $f: G / H \rightarrow G / K$.

The category $\Gamma_{G}=\operatorname{Or}_{\mathcal{F}} G$ is defined as the full subcategory of $\operatorname{Or}(G)$ where the objects satisfy $H \in \mathscr{F}$. The category of right $R \Gamma_{G}$-modules is the category of contravariant functors from $\operatorname{Or}_{\mathcal{F}} G$ to $R$-modules. A right $R \Gamma_{G}$-module $M$ is often called a coefficient system [35]. We will sometimes denote $M(G / H)$ by $M(H)$ if the group $G$ is clear from the context. When $\mathcal{F}=\{e\}, R \Gamma_{G}$-Mod is just the category of left $R G$-modules and Mod- $R \Gamma_{G}$ is just the category of right $R G$-modules.

Now, we will introduce the tensor product and Hom functors for modules over small categories. Let $\Gamma$ be a small category and let $M \in \operatorname{Mod}-R \Gamma$ and $N \in R \Gamma$ Mod. The tensor product over $R \Gamma$ is given by

$$
M \otimes_{R \Gamma} N=\bigoplus_{x \in \mathrm{Ob}(\Gamma)} M(x) \otimes N(x) / \sim
$$


where $\sim$ is the equivalence relation defined by $\varphi^{*}(m) \otimes n \sim m \otimes \varphi_{*}(n)$ for every morphism $\varphi: x \rightarrow y$. For $R \Gamma$-modules $M$ and $N$, we mean by $\operatorname{Hom}_{R \Gamma}(M, N)$ the $R$-module of $R \Gamma$-homomorphisms from $M$ to $N$. In other words,

$$
\operatorname{Hom}_{R \Gamma}(M, N) \subseteq \bigoplus_{x \in \mathrm{Ob}(\Gamma)} \operatorname{Hom}_{R}(M(x), N(x))
$$

is the submodule satisfying the relations $f(x) \circ \varphi^{*}=\varphi^{*} \circ f(y)$, for every morphism $\varphi: x \rightarrow y$. We sometimes consider a second tensor product, namely the tensor product over $R$, which is defined for $R \Gamma$-modules $M$ and $N$ which are both left modules or both right modules. The tensor product $M \otimes_{R} N$ is defined by the formula

$$
\left[M \otimes_{R} N\right](x)=M(x) \otimes_{R} N(x)
$$

on objects $x \in \mathrm{Ob}(\Gamma)$ and on morphisms, one has $\left[M \otimes_{R} N\right](f)=M(f) \otimes_{R} N(f)$.

The tensor product over $R \Gamma$ and $\operatorname{Hom}_{R}$ are adjoint to each other. This can be described in the following way:

Proposition 2.2. Given two small categories $\Gamma$ and $\Lambda$, the category of $R \Gamma-R \Lambda$ bimodules is defined as the category of functors $\Gamma \times \Lambda^{\mathrm{op}} \rightarrow R$-Mod. For a right $R \Gamma$-module $M$, an $R \Gamma$ - $R \Lambda$-bimodule $B$, and a right $R \Lambda$-module $N$, one has a natural transformation

$$
\operatorname{Hom}_{R \Lambda}\left(M \otimes_{R \Gamma} B, N\right) \cong \operatorname{Hom}_{R \Gamma}\left(M, \operatorname{Hom}_{R \Lambda}(B, N)\right) .
$$

Proof. See 9.21 in [20].

We will be using this isomorphism later when we are discussing induction and restriction.

2A. Free and finitely generated modules. For a small category $\Gamma$, a sequence

$$
M^{\prime} \rightarrow M \rightarrow M^{\prime \prime}
$$

of $R \Gamma$-modules is exact if and only if

$$
M^{\prime}(x) \rightarrow M(x) \rightarrow M^{\prime \prime}(x)
$$

is exact for all $x \in \mathrm{Ob}(\Gamma)$. Recall that a module $P$ in $\operatorname{Mod}-R \Gamma$ is projective if the functor

$$
\operatorname{Hom}_{R \Gamma}(P,-): \operatorname{Mod}-R \Gamma \rightarrow R \text {-Mod }
$$

is exact. For an object $x \in \Gamma$, we define a right $R \Gamma$-module $R \Gamma(?, x)$ by setting

$$
R \Gamma(?, x)(y)=R \operatorname{Mor}(y, x)
$$


for all $y \in \operatorname{Ob}(\Gamma)$. Here, $R \operatorname{Mor}(y, x)$ denotes the free abelian group on the set of morphisms $\operatorname{Mor}(y, x)$ from $y$ to $x$. As a consequence of the Yoneda lemma, we have

$$
\operatorname{Hom}_{R \Gamma}(R \Gamma(?, x), M) \cong M(x) .
$$

So, for each $x \in \mathrm{Ob}(\Gamma)$, the module $R \Gamma(?, x)$ is a projective module. When working with modules over small categories one uses the following notion of free modules.

Definition 2.3. Let $\Gamma$ be a small category. $\mathrm{A} \mathrm{Ob}(\Gamma)$-set is defined as a set $S$ together with a map $\beta: S \rightarrow \mathrm{Ob}(\Gamma)$. We say a $R \Gamma$-module $M$ is free if it is isomorphic to a module of the form

$$
R \Gamma(S)=\bigoplus_{b \in S} R \Gamma(?, \beta(b))
$$

for some $\mathrm{Ob}(\Gamma)$-set $S$. A free module $R \Gamma(S)$ is called finitely generated if the set $S$ is finite.

Note that for every $R \Gamma$-module $M$, there is a free $R \Gamma$-module $R \Gamma(S)$ and a map $f: R \Gamma(S) \rightarrow M$ such that $f$ is surjective. We can take such a free module by choosing a set of generators $S_{x}$ for the $R$-module $M(x)$ for each $x \in \mathrm{Ob}(\Gamma)$, and then taking $S$ as the $\operatorname{Ob}(\Gamma)$-set which has the property $\beta^{-1}(x)=S_{x}$. A free module $R \Gamma(S)$ which maps surjectively on $M$ is called a free cover of $M$. A $R \Gamma$-module is called finitely generated if it has a finitely generated free cover.

It is clear from our description of free modules that an $R \Gamma$-module $M$ is projective if and only if it is a direct summand of a free module. This shows that the module category of a small category has enough projectives. We will later give a more detailed description of projective $R \Gamma$-modules.

Example 2.4. For the orbit category $\Gamma=\operatorname{Or}(G)$, the free modules described above have a more specific meaning. For any subgroup $K \leq G$, the $R \Gamma$-module $R \Gamma(?, G / K)$ is given by

$$
R \Gamma(?, G / K)(G / H)=R \operatorname{Mor}(G / H, G / K)=R\left[(G / K)^{H}\right]
$$

where $R\left[(G / K)^{H}\right]$ is the free abelian group on the set of fixed points of the $H$ action on $G / K$. Because of this, we denote the free module $R \Gamma(?, G / K)$ by $R\left[G / K^{?}\right]$.

If $\mathcal{F}$ is a family of subgroups, and $\Gamma_{G}=\operatorname{Or}_{\mathscr{F}} G$, we obtain free $R \Gamma_{G}$-modules $R\left[G / K^{?}\right]$ by restriction whenever $K \in \mathcal{F}$. The constant $R \Gamma_{G}$-module $\underline{R}$ defined by $\underline{R}(H)=R$, for all $H \in \mathscr{F}$, is just the restriction to $R \Gamma_{G}$ of the module $\underline{R}=$ $R\left[G / G^{?}\right]$. This shows that the constant module $\underline{R}$ is projective if $G \in \mathscr{F}$. More generally, if $K \in \mathscr{F}$, a non-empty fixed set

$$
(G / K)^{H}=\left\{g K \mid g^{-1} H g \subseteq K\right\} \neq \varnothing
$$

implies $H \in \mathcal{F}$, since $\mathcal{F}$ is closed under conjugation and taking subgroups. Therefore, $R\left[G / K^{?}\right](H)=0$ for $H \notin \mathcal{F}$, whenever $K \in \mathcal{F}$. 
2B. Induction and restriction. We now recall the definitions and terminology for these terms presented in Lück [20], 9.15. Let $\Gamma$ and $\Lambda$ be two small categories. Given a covariant functor $F: \Lambda \rightarrow \Gamma$, we define an $R \Lambda-R \Gamma$-bimodule

$$
R(? ?, F(?)): \Lambda \times \Gamma^{\mathrm{op}} \rightarrow R-\mathrm{Mod}
$$

on objects by $(x, y) \rightarrow R \operatorname{Hom}(y, F(x))$. We define the restriction map

$$
\operatorname{Res}_{F}: \operatorname{Mod}-R \Gamma \rightarrow \operatorname{Mod}-R \Lambda
$$

as the composition with $F$. The induction map

$$
\operatorname{Ind}_{F}: \operatorname{Mod}-R \Lambda \rightarrow \operatorname{Mod}-R \Gamma
$$

is defined by

$$
\operatorname{Ind}_{F}(M)(? ?)=M \otimes_{R \Lambda} R(? ?, F(?))
$$

for every $R \Lambda$-module $M$. For every right $R \Gamma$-module $N$, the $R \Lambda$-module

$$
\operatorname{Hom}_{R \Gamma}(R(? ?, F(?)), N)
$$

is the same as the composition $\Lambda \stackrel{F}{\rightarrow} \Gamma \stackrel{N}{\rightarrow} R$-Mod. So, by Proposition 2.2, we can conclude the following:

Proposition 2.5. Induction and restriction are adjoint functors: for any $R \Gamma$-module $M$ and $R \Lambda$-module $N$, there is a natural isomorphism

$$
\operatorname{Hom}_{R \Gamma}\left(\operatorname{Ind}_{F} M, N\right)=\operatorname{Hom}_{R \Lambda}\left(M, \operatorname{Res}_{F} N\right) .
$$

The induction functor respects direct sum, finitely generated, free, and projective but it is not exact in general. The restriction functor is exact but does not respect finitely generated, free, or projective in general.

Now we will define functors which are special cases of the restriction and induction functors. Let $\Gamma$ be a small category. For $x \in \mathrm{Ob}(\Gamma)$, we define $R[x]=R \operatorname{Aut}(x)$ to be the group ring of the automorphism group $\operatorname{Aut}(x)$ and denote the category of right $R[x]$-modules by Mod- $R[x]$. Let $\Gamma_{x}$ denote the full subcategory of $\Gamma$ with single object $x$ and let $F: \Gamma_{x} \rightarrow \Gamma$ be the inclusion natural transformation. The restriction functor associated to $F$ gives a functor

$$
\operatorname{Res}_{x}: \operatorname{Mod}-R \Gamma \rightarrow \operatorname{Mod}-R[x]
$$

which is called the restriction functor. This functor behaves like an evaluation map $\operatorname{Res}_{x}(M)=M(x)$. In the other direction, the induction functor associated to $F$ gives a functor

$$
E_{x}: \operatorname{Mod}-R[x] \rightarrow \operatorname{Mod}-R \Gamma
$$


which is called the extension functor. For a $R[x]$-module $M$, we define $E_{x}(M)(y)=$ $M \otimes_{R[x]} R \operatorname{Mor}(y, x)$ for every $y \in \operatorname{Ob}(\Gamma)$. They form an adjoint pair: for every $R[x]$-module $M$ and an $R \Gamma$-module $N$, we have

$$
\operatorname{Hom}_{R \Gamma}\left(E_{x} M, N\right) \cong \operatorname{Hom}_{R[x]}\left(M, \operatorname{Res}_{x} N\right) .
$$

By general properties of restriction and induction, the functor $\operatorname{Res}_{x}$ is exact and $E_{x}$ takes projectives to projectives. In general, $E_{x}$ is not exact and $\operatorname{Res}_{x}$ does not take projectives to projectives. But in some special cases, we can say more. For example, when $\Gamma$ is free, i.e. $R \operatorname{Mor}(y, x)$ is a free $R[x]$-module for all $y \in \Gamma$, then it is easy to see that $E_{x}$ is exact [20], 16.9.

Example 2.6. In the case of an orbit category $\Gamma_{G}=\operatorname{Or}_{\mathcal{F}} G$, we denote the extension function for $H \in \mathcal{F}$ simply by $E_{H}$ and the restriction functor by $\operatorname{Res}_{H}$. In this case, the automorphism group $\operatorname{Aut}(G / H)$ for $H \in \mathscr{F}$ is isomorphic to the quotient group $N_{G}(H) / H$. The isomorphism $N_{G}(H) / H \cong \operatorname{Aut}(G / H)$ is given by the isomorphism $n H \rightarrow f_{n}$ where $f_{n}(g H)=g n^{-1} H$ for $n \in N_{G}(H)$ (see [10], Example 11.2). This isomorphism takes right $R[x]$-modules to right $R\left[N_{G}(H) / H\right]$ modules, so given a right $R \Gamma$-module $M$, the evaluation at $H \in \mathcal{F}$ gives a right $R\left[N_{G}(H) / H\right]$-module.

It is easy to see that the morphism set $\operatorname{Mor}(G / K, G / H)$ is a free $\left[N_{G}(H) / H\right]$-set, so $\operatorname{Or}_{\mathcal{F}} G$ is free in the above sense ([20], Example 16.2). Therefore, the functor $E_{H}$ is exact and preserves projectives, whereas $\operatorname{Res}_{H}$ is exact but does not necessarily preserve projectives. For example, the module $\mathbb{Z}\left[G / G^{?}\right]$ is free over $\mathbb{Z} \operatorname{Or}(G)$ by definition, but $\operatorname{Res}_{H} \mathbb{Z}\left[G / G^{?}\right]=\mathbb{Z}$ is not projective whenever $N_{G}(H) / H \neq 1$.

2C. Inclusion and splitting functors. We will introduce two more functors. These are also special cases of induction and restriction, but they are defined through a bimodule rather than just a natural transformation $F$. We first describe these functors and then give their interpretations as restriction and induction functors.

Let $\Gamma$ be an EI-category. By this, we mean that $\Gamma$ is a small category where every endomorphism $x \rightarrow x$ is an isomorphism for all $x \in \mathrm{Ob}(\Gamma)$. This allows us to define a partial ordering on the set Iso $(\Gamma)$ of isomorphism classes $\bar{x}$ of objects $x$ in $\Gamma$. For $x, y \in \mathrm{Ob}(\Gamma)$, we say $\bar{x} \leq \bar{y}$ if and only if $\operatorname{Mor}(x, y) \neq \emptyset$. The EI-property ensures that $\bar{x} \leq \bar{y} \leq \bar{x}$ implies $\bar{x}=\bar{y}$.

For each object $x \in \Gamma$, and $M \in \operatorname{Mod}-R[x]$, the inclusion functor,

$$
I_{x}: \operatorname{Mod}-R[x] \rightarrow \operatorname{Mod}-R \Gamma
$$

is defined by

$$
I_{x} M(y)= \begin{cases}M \otimes_{R[x]} R \operatorname{Mor}(y, x) & \text { if } \bar{y}=\bar{x} \\ \{0\} & \text { if } \bar{y} \neq \bar{x}\end{cases}
$$


In the other direction, we define the splitting functor

$$
S_{x}: \operatorname{Mod}-R \Gamma \rightarrow \operatorname{Mod}-R[x]
$$

by $S_{x}(M)=M(x) / M(x)_{s}$ where $M(x)_{s}$ is the $R$-submodule of $M(x)$ which is generated by the images of $M(f): M(y) \rightarrow M(x)$ for all $f: x \rightarrow y$ with $\bar{x} \leq \bar{y}$ and $\bar{x} \neq \bar{y}$.

There is a $R \Gamma-R[x]$-bimodule $B$ defined in such a way that the inclusion functor $I_{x}$ can be described as $M \rightarrow \operatorname{Hom}_{R[x]}(B, M)$ and the splitting functor $S_{x}$ is the same as the functor $M \rightarrow M \otimes_{R \Gamma} B$ (see [20], p. 171, for details). So $\left(S_{x}, I_{x}\right)$ is an adjoint pair, meaning that

$$
\operatorname{Hom}_{R[x]}\left(S_{x} M, N\right) \cong \operatorname{Hom}_{R \Gamma}\left(M, I_{x} N\right)
$$

for every $R \Gamma$-module $M$ and $R[x]$-module $N$.

From general properties of induction and restriction, we can conclude that $I_{x}$ is exact and $S_{x}$ preserves projectives. Some of the other properties of these functors are listed in Lemma 9.31 of [20]. It is interesting to note that the composition $S_{x} \circ E_{x}$ is naturally equivalent to the identity functor. Also, the composition $S_{y} \circ E_{x}$ is zero when $\bar{x} \neq \bar{y}$. These are used to give a splitting for projective $R \Gamma$-modules.

Theorem 2.7. Let $P$ be a finitely generated projective $R \Gamma$-module. Then

$$
P \cong \bigoplus_{x \in \operatorname{Iso}(\Gamma)} E_{x} S_{x}(P) .
$$

Proof. For the proof see [20], Corollary 9.40.

In the statement, the notation $\bigoplus_{x \in \operatorname{Iso}(\Gamma)}$ means that the sum is over a set of representatives $x \in \operatorname{Ob}(\Gamma)$ for $\bar{x} \in \operatorname{Iso}(\Gamma)$.

2D. Resolutions for $\boldsymbol{R} \boldsymbol{\Gamma}$-modules. Let $\Gamma$ be an EI-category. For a non-negative integer $l$ we define an $l$-chain $c$ from $x \in \mathrm{Ob}(\Gamma)$ to $y \in \mathrm{Ob}(\Gamma)$ to be a sequence

$$
c: \bar{x}=\bar{x}_{0}<\bar{x}_{1}<\cdots<\bar{x}_{l}=\bar{y} .
$$

Define the length $l(y)$ of $y \in \mathrm{Ob}(\Gamma)$ to be the largest integer $l$ such that there exists an $l$-chain from some $x \in \mathrm{Ob}(x)$ to $y$. The length $l(\Gamma)$ of $\Gamma$ is $\max \{l(x) \mid x \in \mathrm{Ob}(\Gamma)\}$. Given an $R \Gamma$-module $M$, its length $l(M)$ is defined by $\max \{l(x) \mid M(x) \neq 0\}$ if $M$ is not the zero module and $l(\{0\})=-1$.

We call a category $\Gamma$ finite if $\operatorname{Iso}(\Gamma)$ and $\operatorname{Mor}(x, y)$ are finite for all $x, y \in \mathrm{Ob}(\Gamma)$. Denote by $m(\Gamma)$ the least common multiple of all the integers $|\operatorname{Aut}(x)|$.

Given an $R \Gamma$-module $M$, consider the map

$$
\phi: \bigoplus_{x \in \operatorname{Iso}(\Gamma)} E_{x} \operatorname{Res}_{x} M \rightarrow M
$$


where for each $x \in \operatorname{Ob}(\Gamma)$, the map $\phi_{x}: E_{x} \operatorname{Res}_{x} M \rightarrow M$ is the map adjoint to the identity homomorphism. It is easy to see that $\phi$ is surjective. Let

$$
E M:=\bigoplus_{x \in \operatorname{Iso}(\Gamma)} E_{x} \operatorname{Res}_{x} M
$$

and let $K M$ denote the kernel of $\phi: E M \rightarrow M$. Note that if $x$ is an object with $l(x)=l(M)$, then $\operatorname{Res}_{x}=S_{x}$ which also gives that

$$
\operatorname{Res}_{x} \phi: \operatorname{Res}_{x} E_{x} \operatorname{Res}_{x} M \rightarrow \operatorname{Res}_{x} M
$$

is an isomorphism. Note that this implies $l(K M)<l(M)$ which allows one to proceed by induction and obtain the following:

Proposition 2.8. If $\Gamma$ is a finite EI-category, then every nonzero $M$ has a finite resolution of the form

$$
0 \rightarrow E K^{t} M \rightarrow \cdots \rightarrow E K M \rightarrow E M \rightarrow M \rightarrow 0
$$

where $t=l(M)$.

Proof. See [20], 17.13. Here $K^{0} M=M$ and $K^{s} M=K\left(K^{s-1} M\right)$.

From the description above it is easy to see that

$$
E K^{s} M:=\bigoplus_{x \in \operatorname{Iso}(\Gamma)} E_{x} \operatorname{Res}_{x} K^{s} M
$$

where $\operatorname{Res}_{x} K^{s} M$ is isomorphic to a direct sum of $R[x]$-modules

$$
M(c):=M\left(x_{0}\right) \otimes_{R\left[x_{0}\right]} R \operatorname{Mor}\left(x_{1}, x_{0}\right) \otimes_{R\left[x_{1}\right]} \cdots \otimes_{R\left[x_{s-1}\right]} R \operatorname{Mor}\left(x, x_{s-1}\right)
$$

over representatives in $\mathrm{Ob}(\Gamma)$ for all the chains of the form $c: \bar{x}<\bar{x}_{s-1}<\cdots<\bar{x}_{0}$ (see [20], 17.24). Note that if $\Gamma$ is a finite, free EI-category, then the resolution given in Proposition 2.8 will be a finite projective resolution if $M(c)$ is projective as an $R[x]$-module for every chain $c$. This gives the following:

Proposition 2.9. Let $M$ be $R \Gamma_{G}$ module where $\Gamma_{G}=\mathrm{Or}_{\mathcal{F}} G$ for some finite group $G$ and $R$ is a commutative ring such that $|G|$ is invertible in $R$. Suppose also that $M(H)$ is projective as an $R$-module for all $H \in \mathcal{F}$. Then, $M$ has a projective resolution with length less than or equal to $l(\Gamma)$.

Proof. See [20], 17.31.

In particular, if $R=\mathbb{Z}_{(p)}$ with $p \nmid|G|$ and if $M$ is a $R \Gamma$-module such that $M(H)$ is $R$-torsion free for all $H \in \mathcal{F}$, then $M$ has a finite projective resolution of length less than or equal to $l(M)$. 


\section{The proof of Theorem B}

The main result of this section is Theorem 3.9, which is an orbit category version of a well-known result of Rim [29]. We first collect some further information about induction and restriction for subgroups.

Let $G$ be a finite group and let $H$ be a subgroup of $G$. Given a family of subgroups $\mathcal{F}$ of $G$, we consider the orbit categories $\Gamma_{G}=\operatorname{Or}_{\mathscr{F}} G$ and $\Gamma_{H}=\operatorname{Or}_{\mathscr{F}} H$, where the objects of $\Gamma_{H}$ are orbits of $H$ with isotropy in $\widetilde{F}_{H}=\{K \leq H \mid K \in \mathscr{F}\}$. Let $F: \Gamma_{H} \rightarrow \Gamma_{G}$ be the functor which takes $H / K$ to $G / K$ and sends an $H$-map $f: H / K \rightarrow H / L$ to the induced $G$-map

$$
\operatorname{Ind}_{H}^{G}(f): G / K=G \times_{H} H / K \rightarrow G \times_{H} H / L=G / L
$$

for every $K, L \in \mathcal{F}_{H}$. Note that if $f$ is the map which takes $e K$ to $h L$, then $\operatorname{Ind}_{H}^{G}(f)(g K)=g h L$. The restriction and induction functors (see Proposition 2.5) associated to this functor gives us two adjoint functors

$$
\operatorname{Res}_{H}^{G}: \operatorname{Mod}-\Gamma_{G} \rightarrow \operatorname{Mod}-\Gamma_{H}
$$

and

$$
\operatorname{Ind}_{H}^{G}: \operatorname{Mod}-\Gamma_{H} \rightarrow \operatorname{Mod}-\Gamma_{G} .
$$

The restriction functor is defined as the composition with $F$. So, for a $R \Gamma_{G}$-module $M$, we have $\left(\operatorname{Res}_{H}^{G} M\right)(K)=M(K)$, for all $K \in \mathcal{F}_{H}$. For the induced module we have the following formula:

Lemma 3.1. Let $N$ be a $R \Gamma_{H}$-module and $K \leq G$. Then,

$$
\left(\operatorname{Ind}_{H}^{G} N\right)(K) \cong \bigoplus_{g H \in G / H, K^{g} \leq H} N\left(K^{g}\right)
$$

where $K^{g}=g^{-1} \mathrm{Kg}$.

Proof. For a (right) $R \Gamma_{H}$-module $N$, the induced module $\operatorname{Ind}_{H}^{G} N$ is defined as the direct sum

$$
\bigoplus_{L \leq H} N(L) \otimes_{R} R \operatorname{Mor}(G / K, G / L)
$$

modulo the relations $n \otimes \varphi f \sim \varphi^{*}(n) \otimes f$ where $n \in N(L), f \in \operatorname{Mor}\left(G / K, G / L^{\prime}\right)$ and $\varphi=\operatorname{Ind}_{H}^{G}(\phi)$ for some $\phi: H / L^{\prime} \rightarrow H / L$. Every morphism $G / K \rightarrow G / L$ which satisfies the condition $L \leq H$ can be written as a composition $\varphi f_{g}$ where $\varphi: G / K^{g} \rightarrow G / L$ is induced from an $H$-map and $f_{g}: G / K \rightarrow G / K^{g}$ is given by $x K \rightarrow x g K^{g}$, for some $g \in G$.

This shows that every element in the above sum is equivalent to an element of the form $n \otimes f_{g}$ where $n \in N\left(K^{g}\right)$ and $f_{g}: G / K \rightarrow G / K^{g}$ is as above with $K^{g} \leq H$. There is one summand for each $g H$ satisfying $K^{g} \leq H$. 
Note that we can also express the above formula by

$$
\left.\operatorname{Ind}_{H}^{G} N\right)(K) \cong \bigoplus_{g H \in(G / H)^{K}} N\left(K^{g}\right) .
$$

If $J \leq K$, then the argument above can be extended to show that restriction map

$$
\left(\operatorname{Ind}_{H}^{G} N\right)(K) \rightarrow\left(\operatorname{Ind}_{H}^{G} N\right)(J)
$$

is given by the coordinate-wise restriction maps $N\left(K^{g}\right) \rightarrow N\left(J^{g}\right)$. Note that if $g H \in(G / H)^{K}$, then $g H \in(G / H)^{J}$. Similarly, the conjugation map

$$
\left(\operatorname{Ind}_{H}^{G} N\right)(K) \rightarrow\left(\operatorname{Ind}_{H}^{G} N\right)\left({ }^{x} K\right)
$$

can be described by coordinate-wise conjugation maps. From these, it is easy to see that $\operatorname{Ind}_{H}^{G} \underline{R} \cong R\left[G / H^{?}\right]$. A generalization of this argument gives the following:

Lemma 3.2 ([35], Corollary 2.12). Let $G$ be a finite group and let $H$ be a subgroup of $G$. For every $R \Gamma_{G}$-module $M$, we have $\operatorname{Ind}_{H}^{G} \operatorname{Res}_{H}^{G} M \cong M \otimes_{R} R\left[G / H^{?}\right]$.

We also have the following formulas:

Lemma 3.3. Let $G$ be a finite group and let $H$ be a subgroup of $G$.

(i) For every $K \leq H$, we have $\operatorname{Ind}_{H}^{G} R\left[H / K^{?}\right] \cong R\left[G / K^{?}\right]$.

(ii) For every $K \leq G$, we have $\operatorname{Res}_{H}^{G} R\left[G / K^{?}\right] \cong \bigoplus_{K \backslash G / H} R\left[H /\left(H \cap{ }^{g} K\right)^{?}\right]$.

Proof. Part (i) follows from the fact that $\operatorname{Ind}_{H}^{G} \operatorname{Ind}_{K}^{H}=\operatorname{Ind}_{K}^{G}$ which is a consequence of a more general formula $\operatorname{Ind}_{F} \operatorname{Ind}_{F^{\prime}}=\operatorname{Ind}_{F \circ F^{\prime}}$. We can prove this more general formula by using adjointness and the formula $\operatorname{Res}_{F^{\prime}} \operatorname{Res}_{F}=\operatorname{Res}_{F \circ F^{\prime}}$. For (ii), observe that the definition of $R\left[G / H^{?}\right]$ can be extended to define a $R \Gamma_{G}$-module $R\left[S^{?}\right]$ for every $G$-set $S$, by taking

$$
R\left[S^{?}\right](G / K)=R \operatorname{Map}_{G}(G / K, S)
$$

for every $K \in \mathscr{F}$, where $\operatorname{Map}_{G}(G / K, S)$ denotes the set of $G$-maps from $G / K$ to $S$. For $G$-sets $S$ and $T$, we have an isomorphism $R\left[(S \sqcup T)^{?}\right] \cong R\left[S^{?}\right] \oplus R\left[T^{?}\right]$. By the definition of restriction map, we get

$$
\left(\operatorname{Res}_{H}^{G} R\left[S^{?}\right]\right)(H / K)=R \operatorname{Map}_{G}(G / K, S)=R \operatorname{Map}_{H}\left(H / K, \operatorname{Res}_{H}^{G} S\right) .
$$

It is easy to see that this induces an $R \Gamma_{H}$-module isomorphism

$$
\operatorname{Res}_{H}^{G} R\left[S^{?}\right] \cong R\left[\left(\operatorname{Res}_{H}^{G} S\right)^{?}\right] \text {. }
$$

Since

$$
\operatorname{Res}_{H}^{G}(G / K) \cong \coprod_{H \backslash G / K} H /\left(H \cap{ }^{g} K\right)
$$

as $G$-sets, we obtain the formula given in (ii). 
Example 3.5. Let $G=S_{5}$ be the symmetric group on $\{1,2,3,4,5\}$ and $H=S_{4}$ be the subgroup of symmetries that fix 5. Let $C_{2}=\langle(12)\rangle$ and $C_{3}=\langle(345)\rangle$. The formula in Lemma 3.3 (ii) gives

$$
\operatorname{Res}_{H}^{G} R\left[G /\left(C_{2} \times C_{3}\right)^{?}\right]=R\left[H / C_{2}{ }^{?}\right] \oplus R\left[H /{ }^{g} C_{3}{ }^{?}\right]
$$

where ${ }^{g} C_{3}=\langle(123)\rangle$. From this expression we obtain

$$
R\left[G /\left(C_{2} \times C_{3}\right)^{?}\right]\left(G / C_{2}\right) \cong R\left[H / C_{2}{ }^{?}\right]\left(H / C_{2}\right) \cong R\left[N_{H}\left(C_{2}\right) / C_{2}\right],
$$

as an $N_{H}\left(C_{2}\right) / C_{2}$-module, where $N_{H}\left(C_{2}\right)=C_{2} \times C_{2}$. Note that $N_{G}\left(C_{2}\right)=$ $C_{2} \times S_{3}$ and as an $N_{G}\left(C_{2}\right) / C_{2}$-module $R\left[G /\left(C_{2} \times C_{3}\right)^{?}\right]\left(G / C_{2}\right)$ is isomorphic to $R\left[C_{2} \times S_{3} / C_{2} \times C_{3}\right]$.

We can give a more general formula for $R\left[G / H^{?}\right](G / K)$ as follows:

Lemma 3.6. Let $G$ be a finite group, and $H$ and $K$ be two subgroups of $G$. Then, as an $R\left[N_{G}(H) / H\right]$-module

$$
R\left[G / K^{?}\right](G / H) \cong \bigoplus_{v(H, K)} R\left[N_{G}(H) / N g_{K}(H)\right]
$$

where the sum is over the set $v(H, K)$ of representatives of $K$-conjugacy classes of subgroups $H^{g}$ such that $H^{g} \leq K$.

Proof. This formula can easily be proved by first determining the orbits of $N_{G}(H)$ action on $(G / K)^{H}=\left\{g K \mid H^{g} \leq K\right\}$, and then by calculating the isotropy subgroups for each of these orbits. A similar computation can be found in the proof of Theorem 4.1 in [8].

Proposition 3.7. Both $\operatorname{Res}_{H}^{G}$ and $\operatorname{Ind}_{H}^{G}$ are exact and take projectives to projectives.

Proof. The fact that $\operatorname{Res}_{H}^{G}$ is exact and $\operatorname{Ind}_{H}^{G}$ preserves projectives follows from the general properties of restriction and induction functor associated to a natural transformation $F$. The fact that $\operatorname{Ind}_{H}^{G}$ is exact follows from the formula given in Lemma 3.1. Finally, to show that $\operatorname{Res}_{H}^{G}$ takes projective to projectives, it is enough to show it takes free modules to projective modules. An indecomposable free $R \Gamma_{G}$-module $M$ is of the form $R\left[G / K^{?}\right]$ for some $K \in \mathscr{F}$. By Lemma 3.3, $\operatorname{Res}_{H}^{G}\left(R\left[G / K^{?}\right]\right)$ will be projective if $H \cap{ }^{g} K$ is in $\mathscr{F}$ for all $H g K \in H \backslash G / K$. But this is always true since the family $\mathscr{F}$ is closed under conjugation and taking subgroups.

A result of $\operatorname{Rim}[29]$ relates projectivity over the group ring $\mathbb{Z} G$ to projectivity over the $p$-Sylow subgroups. 
Proposition 3.8 (Rim's theorem). Let $G$ be a finite group, and $M$ be a finitely generated $\mathbb{Z} G$-module. Then $M$ is projective over $\mathbb{Z} G$ if and only if $\operatorname{Res}_{P}^{G} M$ is projective over $\mathbb{Z} P$ for any $p$-Sylow subgroup $P \leq G$.

Proof. A module $M$ is $\mathbb{Z} G$-projective if and only if $\operatorname{Ext}_{\mathbb{Z} G}^{1}(M, N)=0$ for every $\mathbb{Z} G$-module $N$. Therefore $M$ is projective if and only if $\mathbb{Z}_{(p)} \otimes_{\mathbb{Z}} M$ is projective over $\mathbb{Z}_{(p)} G$ for all primes $p$ dividing the order of $G$.

For any $p$-Sylow subgroup $P \leq G$, the permutation module $R[G / P] \cong R \oplus N$ splits when $R=\mathbb{Z}_{(p)}$. Therefore, if $M$ is any $R G$-module, $M \otimes_{R} R[G / P] \cong$ $M \oplus\left(M \otimes_{R} N\right)$. Since $M \otimes_{R} R[G / P] \cong \operatorname{Ind}_{P}^{G} \operatorname{Res}_{P}^{G} M$, the projectivity of $M$ is equivalent to the projectivity of $\operatorname{Res}_{P}^{G} M$.

Here is an orbit category version of this result.

Theorem 3.9 (Rim's theorem for the orbit category). Let $G$ be a finite group and let $M$ be a $R \Gamma_{G}$-module where $R=\mathbb{Z}_{(p)}$. Suppose that $\mathcal{F}_{\mathcal{F}}$ is a family of $p$-subgroups in $G$. Then $M$ has a finite projective resolution if and only if $\operatorname{Res}_{P}^{G} M$ has a finite projective resolution for any $p$-Sylow subgroup $P$ of $G$.

Proof. One direction is clear since $\operatorname{Res}_{P}^{G}$ is exact and takes projectives to projectives. For the other direction, we will give the proof by induction on the length $l(M)$ of $M$. Without loss of generality, we can assume that $M(H)$ is $R$-torsion free for all $H \in \mathcal{F}$. Suppose $M$ is a $R \Gamma_{G}$-module with $l(M)=0$. Then, we can regard $M$ as an $R G$-module. If $\operatorname{Res}_{P}^{G} M$ has a finite projective resolution, then $\operatorname{Res}_{P}^{G} M$ must be projective (see [20], p. 348). Then, by Rim's theorem, $M$ is a projective $R G$-module, hence has finite projective length.

Now, assume $M$ is an $R \Gamma_{G}$-module with $l(M)=s>0$. Let

$$
0 \rightarrow P_{n} \rightarrow \cdots \rightarrow P_{0} \rightarrow \operatorname{Res}_{P}^{G} M \rightarrow 0
$$

be a projective resolution for $\operatorname{Res}_{P}^{G} M$. We can assume that $l\left(P_{i}\right) \leqslant s$ for all $i$. Then, for $Q \in \widetilde{F}$ with $l(Q)=s$, we have

$$
S_{Q} P_{i}=\operatorname{Res}_{Q} P_{i}=P_{i}(Q) .
$$

Since $S_{Q}$ takes projectives to projectives, the resolution

$$
0 \rightarrow P_{n}(Q) \rightarrow \cdots \rightarrow P_{0}(Q) \rightarrow\left(\operatorname{Res}_{P}^{G} M\right)(Q) \rightarrow 0
$$

is a finite projective resolution of $\left(\operatorname{Res}_{P}^{G} M\right)(Q)=M(Q)$ as an $R\left[N_{P}(Q) / Q\right]$ module. This gives that $M(Q)$ is projective as an $R\left[N_{P}(Q) / Q\right]$-module.

Lemma 3.10. For every $p$-group $Q$, there is a $p$-Sylow subgroup $P$ of $G$ such that $N_{P}(Q)$ is a $p$-Sylow subgroup of $N_{G}(Q)$. 
Proof. Let $S$ be a $p$-Sylow subgroup of $N_{G}(Q)$, and pick a $p$-Sylow subgroup $P$ of $G$ containing $S$. Since $N_{P}(Q)=N_{G}(Q) \cap P$ is a $p$-subgroup of $N_{G}(Q)$, we have $\left|N_{P}(Q)\right| \leq|S|$. But $S \leq P$ and $S \leq N_{G}(Q)$ implies $S \leq N_{P}(Q)$. Therefore $S=N_{P}(Q)$.

We can assume $P$ is a $p$-Sylow subgroup which has this property. Then, by the $p$-local version of Rim's theorem, we can conclude that $M(Q)$ is projective as an $R\left[N_{G}(Q) / Q\right]$-module. Now, consider the map

$$
\psi=\left(\psi_{Q}\right): \bigoplus_{Q \in \operatorname{Iso}\left(\Gamma_{G}\right), l(Q)=s} E_{Q} \circ \operatorname{Res}_{Q} M \rightarrow M
$$

where $\psi_{Q}: E_{Q} \circ \operatorname{Res}_{Q} M \rightarrow M$ is the map adjoint to the identity mapid: $\operatorname{Res}_{Q} M \rightarrow$ $\operatorname{Res}_{Q} M$. For every $K \in \mathcal{F}$ with $l(K)=s$, the induced map $\psi(K)$ is an isomorphism. This is because

$$
\left(E_{Q} \circ \operatorname{Res}_{Q} M\right)(K)=\operatorname{Res}_{K} E_{Q} \operatorname{Res}_{Q} M=S_{K} E_{Q} \operatorname{Res}_{Q} M \cong M(K)
$$

if $K$ is conjugate to $Q$ and zero otherwise. So, we have $l(\operatorname{coker} \psi)<s$. Therefore, there is a finitely generated projective $R \Gamma_{G}$-module $P$ with $l(P)<s$, and a map $\alpha: P \rightarrow M$ such that $\psi \oplus \alpha$ is surjective. If $K$ is the kernel of $\psi \oplus \alpha$, we get an exact sequence of $R \Gamma_{G}$-modules

$$
0 \rightarrow K \rightarrow P \oplus \bigoplus_{Q \in \operatorname{Iso}\left(\Gamma_{G}\right), l(Q)=s} E_{Q} \circ \operatorname{Res} Q M \rightarrow M \rightarrow 0
$$

where the middle term is projective as an $R \Gamma_{G}$-module, and $l(K)<s$. Note that $\operatorname{Res}_{P}^{G} K$ must have a finite projective resolution by Lemma 11.6 of [20]. So, by induction, $K$ has a finite projective resolution, and hence $M$ has a finite projective resolution as well.

Remark 3.11. The inductive argument we use in the above proof is similar to the argument used by Lück to prove Proposition 17.31 in [20]. By this result, any module $M$ over a finite EI-category $\Gamma$ which has a finite projective resolution, admits a resolution of length $\leqslant l(M)$ provided that $M(x)$ is $R$-projective for all $x \in \mathrm{Ob}(\Gamma)$.

It is not clear to us how to generalize Theorem 3.9 to integer coefficients. For $R=\mathbb{Z}_{(p)}$, the following example shows that the result does not hold for an arbitrary family $\mathcal{F}$.

Example 3.12. Let $G=S_{5}$ and $R=\mathbb{Z}_{(2)}$, and take $\mathscr{F}$ as the family of all 2-subgroups and 3-subgroups in $G$. Consider the $R \Gamma_{G}$-module $M=R\left[G /\left(C_{2} \times C_{3}\right)^{?}\right.$ ] 
where $C_{2}$ and $C_{3}$ are as in Example 3.5. It is clear that the restriction of $M$ to a 2Sylow subgroup is projective (since its restriction to $H=S_{4}$ is already projective), but $M$ does not have a finite projective resolution as an $R \Gamma_{G}$-module.

To see this, suppose that $M$ has a finite projective resolution $\mathbf{P} \rightarrow M$. Then, $\mathbf{P}\left(C_{3}\right)$ will be a finite projective resolution for $M\left(C_{3}\right)$ over $R\left[N_{G}\left(C_{3}\right) / C_{3}\right]$. This is because $C_{3}=\langle(123)\rangle$ is a maximal subgroup in $\mathscr{F}$. This implies

$$
M\left(C_{3}\right) \cong R\left[S_{3} \times C_{2} / C_{3} \times C_{2}\right] \cong R\left[C_{2}\right]
$$

is projective as an $R\left[N_{G}\left(C_{3}\right) / C_{3}\right]$-module. But,

$$
R\left[N_{G}\left(C_{3}\right) / C_{3}\right]=R\left[S_{3} \times C_{2} / C_{3}\right] \cong R\left[C_{2} \times C_{2}\right],
$$

and it is clear that $R\left[C_{2}\right]$ is not projective as an $R\left[C_{2} \times C_{2}\right]$-module. So, $M$ does not have a finite projective resolution.

On the other hand, the following holds for modules over orbit categories:

Proposition 3.13. Let $G$ be a finite group, and $\mathcal{F}$ be a family of subgroups of $G$. Then, a $\mathbb{Z} \Gamma_{G}$-module $M$ has a finite projective resolution if and only if $\mathbb{Z}_{(p)} \otimes_{\mathbb{Z}} M$ has a finite projective resolution over $\mathbb{Z}_{(p)} \Gamma_{G}$, for all primes $p$ dividing the order of $G$.

The proof of this statement follows from Propositions 4.4 and 4.5 in the next section. We end this section with some corollaries of Theorem 3.9.

Corollary 3.14. Let $G$ be a finite group and $R=\mathbb{Z}_{(p)}$. Suppose that $\mathscr{F}$ is a family of p-subgroups. Then, $R\left[G / H^{?}\right]$ has a finite projective resolution over $R \Gamma_{G}$ if a $p$-Sylow subgroup of $H$ is included in $\mathcal{F}$.

Proof. If a $p$-Sylow subgroup of $H$ is in $\mathcal{F}$, then $\operatorname{Res}_{P}^{G} R\left[G / H^{?}\right]$ is a free $R \Gamma_{P}$-module for any $P \in \operatorname{Syl}_{p}(G)$. So, by Theorem 3.9, it has a finite projective resolution.

As a special case of this corollary, we obtain the following known result (see (6.8) in [4], [35] (2.5 and p. 296), [17], [14]).

Corollary 3.15. Let $G$ be a finite group and $R=\mathbb{Z}_{(p)}$. Then, $\underline{R}$ has a finite projective resolution over $R \Gamma_{G}$ relative to the family of all $p$-subgroups of $G$.

Proof. This follows from $\underline{R}=R\left[G / G^{?}\right]$. 


\section{Mackey structures on $\operatorname{Ext}_{R \Gamma_{G}}^{*}(M, N)$}

The notation and results of the previous sections will now be used to establish some structural and computational facts about the Ext-groups over the orbit category. Our main sources are Cartan-Eilenberg [7] and tom Dieck [10], §II.9 (see also [17], [14]).

We have seen that the category of right $R \Gamma$-modules has enough projectives to define the bifunctor

$$
\operatorname{Ext}_{R \Gamma}^{*}(M, N)=H^{*}\left(\operatorname{Hom}_{R \Gamma}(\mathbf{P}, N)\right)
$$

via any projective resolution $\mathbf{P} \rightarrow M$ (see [20], Chapter III, §17, [23], Chapter III.6). The following property is also useful (see Lück [20], 17.21).

Lemma 4.1. If $\Gamma$ is a free EI-category, then

$$
\operatorname{Ext}_{R \Gamma}^{*}\left(E_{x} M, N\right) \cong \operatorname{Ext}_{R[x]}^{*}\left(M, \operatorname{Res}_{x} N\right) .
$$

Proof. Take a projective resolution $\mathbf{P}$ of $M$. Since $\Gamma$ is free, the extension functor $E_{x}$ is exact [20], 16.9. In addition, $E_{x}$ preserves projectives and is adjoint to the restriction functor $\operatorname{Res}_{x}$ by Proposition 2.5. Therefore

$$
\cdots \rightarrow E_{x} P_{n} \rightarrow \cdots \rightarrow E_{x} P_{1} \rightarrow E_{x} P_{0} \rightarrow E_{x} M \rightarrow 0
$$

is a projective resolution of $E_{x} M$, and applying Hom over the orbit category gives

$$
\begin{aligned}
\operatorname{Ext}_{R \Gamma}^{n}\left(E_{x} M, N\right) & =H^{n}\left(\operatorname{Hom}_{R \Gamma}\left(E_{x} \mathbf{P}, N\right)\right) \\
& \cong H^{n}\left(\operatorname{Hom}_{R[x]}\left(\mathbf{P}, \operatorname{Res}_{x} N\right)\right)=\operatorname{Ext}_{R[x]}^{n}\left(M, \operatorname{Res}_{x} N\right) .
\end{aligned}
$$

In the rest of this section, we assume that $\Gamma_{G}=\operatorname{Or}_{\mathscr{F}} G$ for a finite group $G$, where $\mathcal{F}$ is a family of subgroups in $G$. Note that $\Gamma_{G}$ is both finite and free as an EI-category. If there are two groups $H \leq G$, we use the notations $\Gamma_{G}=\operatorname{Or}_{\mathscr{F}} G$ for the orbit category with respect to the family $\mathscr{F}$, and $\Gamma_{H}=\mathrm{Or}_{\mathcal{F}} H$ for the orbit category with respect to the family $\mathscr{F}_{H}=\{H \cap K \mid K \in \mathscr{F}\}$.

Proposition 4.2. Let $M$ and $N$ be two $\mathbb{Z} \Gamma_{G}$-modules, where $M(H)$ is $\mathbb{Z}$-torsion free for all $H \in \mathscr{F}$. Then for every $n>l(M)$, the groups $\operatorname{Ext}_{\mathbb{Z} \Gamma_{G}}^{n}(M, N)$ are finite abelian, with exponent dividing some power of $|G|$.

Proof. This follows from the Lemma 4.1, Proposition 2.8, and the corresponding result for modules over finite groups.

Note that the Ext-groups in lower dimensions are not finite in general. But, it is still true in all dimensions that the Ext-groups over $\mathbb{Z} \Gamma_{G}$ vanish if and only if they 
vanish over $\mathbb{Z}_{(p)} \Gamma_{G}$, for all primes $p$. To see this, we note that tensoring over $\mathbb{Z}$ with $\mathbb{Z}_{(p)}$ preserves exactness, and hence

$$
\operatorname{Ext}_{\mathbb{Z} \Gamma_{G}}^{n}(M, N) \otimes_{\mathbb{Z}} \mathbb{Z}_{(p)} \cong \operatorname{Ext}_{\mathbb{Z}_{(p)} \Gamma_{G}}^{n}\left(M \otimes_{\mathbb{Z}} \mathbb{Z}_{(p)}, N \otimes_{\mathbb{Z}} \mathbb{Z}_{(p)}\right) .
$$

We also have the following:

Proposition 4.4. Let $M$ and $N$ be two $\mathbb{Z} \Gamma_{G}$-modules, where $M(H)$ is $\mathbb{Z}$-torsion free for all $H \in \mathcal{F}$. Then, for every $n>l(M)$, there is an isomorphism

$$
\operatorname{Ext}_{\mathbb{Z} \Gamma_{G}}^{n}(M, N) \cong \bigoplus_{p \| G \mid} \operatorname{Ext}_{\mathbb{Z}_{(p)} \Gamma_{G}}\left(M_{p}, N_{p}\right)
$$

where $M_{p}=\mathbb{Z}_{(p)} \otimes_{\mathbb{Z}} M$ and $N_{p}=\mathbb{Z}_{(p)} \otimes_{\mathbb{Z}} N$.

Proof. From Proposition 4.2 we know that $\operatorname{Ext}_{\mathbb{Z} \Gamma_{G}}^{n}(M, N)$ is a finite abelian group with exponent dividing some power of $|G|$, when $n>l(M)$. Now the flatness of $\mathbb{Z}_{(p)}$ over $\mathbb{Z}$ implies as above that $\operatorname{Ext}_{\mathbb{Z}}^{n} \Gamma_{G}(M, N)$ is the direct sum of its $p$-localizations, for all $p|| G \mid$. We then apply the isomorphism (4.3).

To complete the proof of Proposition 3.13, we also need the following standard result in homological algebra (see [7], Chapter VI, 2.1, for the case of modules over rings):

Proposition 4.5. A right $R \Gamma_{G}$-module $M$ admits a finite projective resolution if and only if there exists an integer $\ell_{0} \geqslant 0$ such that $\operatorname{Ext}_{R \Gamma_{G}}^{n}(M, N)=0$, for all $n>\ell_{0}$ and all right $R \Gamma_{G}$-modules $N$.

Proof. If $M$ admits a finite projective resolution of length $k$, then $\operatorname{Ext}_{R \Gamma_{G}}^{n}(M, N)=0$ for $n>k$ and any $R \Gamma_{G}$-module $N$. Conversely, if $\operatorname{Ext}_{R \Gamma_{G}}^{n}(M, N)=0$ for $n>\ell_{0}$ and any $N$, then consider the kernel $Z_{m}$ of the boundary map $\partial_{m}: P_{m} \rightarrow P_{m-1}$ in the projective resolution $\mathbf{P}$ of $M$. It follows that

$$
\operatorname{Ext}_{R \Gamma_{G}}^{1}\left(Z_{m}, N\right) \cong \operatorname{Ext}_{R \Gamma_{G}}^{m+2}(M, N)=0
$$

for any $R \Gamma_{G}$-module $N$, provided $m+2>\ell_{0}$, and so $Z_{m}$ is projective if we take $m=\ell_{0}-1$. This gives a finite projective resolution of length $\ell_{0}$ over $R \Gamma_{G}$.

We now recall the definition of a Mackey functor (following Dress [13]). Let $G$ be a finite group and $\mathscr{D}(G)$ denote the Dress category of finite $G$-sets and $G$-maps. A bivariant functor

$$
M=\left(M^{*}, M_{*}\right): \mathscr{D}(G) \rightarrow R \text {-Mod }
$$

consists of a contravariant functor

$$
M^{*}: \mathscr{D}(G) \rightarrow R-\operatorname{Mod}
$$


and a covariant functor

$$
M_{*}: \mathscr{D}(G) \rightarrow R \text {-Mod. }
$$

The functors are assumed to coincide on objects. Therefore, we write $M(S)=$ $M_{*}(S)=M^{*}(S)$ for a finite $G$-set $S$. If $f: S \rightarrow T$ is a morphism, we often use the notation $f_{*}=M_{*}(f)$ and $f^{*}=M^{*}(f)$. If $S=G / H$ and $T=G / K$ with $H \leq K$ and $f: G / H \rightarrow G / K$ is given by $f(e H)=e K$, then we use the notation $f_{*}=\operatorname{Ind}_{H}^{K}$ and $f^{*}=\operatorname{Res}_{H}^{K}$.

Definition 4.6 (Dress [13]). A bivariant functor is called a Mackey functor if it has the following properties:

(M1) For each pullback diagram

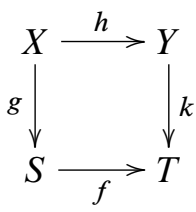

of finite $G$-sets, we have $h_{*} \circ g^{*}=k^{*} \circ f_{*}$.

(M2) The two embeddings $S \rightarrow S \bigsqcup T \longleftarrow T$ into the disjoint union define an isomorphism $M^{*}(S \bigsqcup T) \cong M^{*}(S) \oplus M^{*}(T)$.

Remark 4.7. There is a functor $\operatorname{Or}(G) \rightarrow \mathcal{D}(G)$ defined on objects by $H \mapsto G / H$ for every subgroup $H \leq G$, and as the identity on morphism sets. By composition, any contravariant functor $\mathscr{D}(G) \rightarrow R$-Mod gives a right $R \Gamma_{G}$-module, with respect to any given family of subgroups $\mathcal{F}_{\mathcal{H}}$ of $G$.

In the statement of Theorem 4.11 we will use the examples $R\left[S^{?}\right]: \mathcal{D}(G) \rightarrow$ $R$-Mod, defined in (3.4) for any finite $G$-set $S$.

The following example and lemma will be used in the proof of Theorem 5.9.

Example 4.8. Let $Q \in \mathcal{F}$ and let $V$ be a right $R\left[W_{G}(Q)\right]$-module, where $W_{G}(Q)=$ $N_{G}(Q) / Q$. Then we define a bivariant functor $D_{Q}(V): \mathscr{D}(G) \rightarrow R$-Mod on objects by setting

$$
D_{Q}(V)(S)=\operatorname{Hom}_{R\left[W_{G}(Q)\right]}\left(R\left[S^{Q}\right], V\right)
$$

for any finite $G$-set $S$. For any $G$-map $f: S \rightarrow T$ we have a $W_{G}(Q)$-map $f^{Q}: S Q \rightarrow$ $T^{Q}$, which induces a homomorphism

$$
f^{*}: \operatorname{Hom}_{R\left[W_{G}(Q)\right]}\left(R\left[T^{Q}\right], V\right) \rightarrow \operatorname{Hom}_{R\left[W_{G}(Q)\right]}\left(R\left[S^{Q}\right], V\right)
$$

by composition. To define the covariant map $f_{*}$, let $\varphi_{S}: R\left[S^{Q}\right] \rightarrow V$ be an $R\left[W_{G}(Q)\right]$-homomorphism, and define $f_{*}\left(\varphi_{S}\right)=\varphi_{T}$ by

$$
f_{*}\left(\varphi_{S}\right)(t)=\varphi_{T}(t)=\sum_{s \in S} \varphi_{, f(s)=t}(s)
$$


It is not hard to verify that $D_{Q}(V)$ is actually a Mackey functor. The axiom (M1) follows because the $Q$-fixed sets in a pull-back diagram of $G$-sets give again a pullback diagram. The axiom (M2) is immediate.

Definition 4.9. For any $R \Gamma_{G}$-module $N$, we define $D N=\sum_{Q \in \operatorname{Iso}\left(\Gamma_{G}\right)} D_{Q}(N(Q))$ and define $j: N \rightarrow D N$ as the direct sum of the adjoints of id: $N(Q) \rightarrow N(Q)$, for each $Q \in \operatorname{Iso}\left(\Gamma_{G}\right)$. Let $C N$ denote the cokernel of $j$. For $k \geq 0$, define inductively $C^{0} N=N$ and $C^{k} N=C\left(C^{k-1} N\right)$, together with the induced maps $C^{k} \rightarrow D C^{k}$.

Here is a dual construction to the E-resolution given in [20], 17.13.

Lemma 4.10. For any $R \Gamma_{G}$-module $N$, the finite length sequence

$$
0 \rightarrow N \stackrel{j}{\rightarrow} D N \rightarrow D C N \rightarrow \cdots \rightarrow D C^{m} N \rightarrow 0
$$

is an exact coresolution of Mackey functors, for some $m \geq 0$.

Proof. For any $R \Gamma_{G}$-module $N$, the map $j: N \rightarrow D N$ defined above is injective, so we have a short exact sequence

$$
0 \rightarrow N \stackrel{j}{\rightarrow} D N \rightarrow C N \rightarrow 0
$$

Iterating the above process, we obtain

$$
0 \rightarrow C N \rightarrow D C N \rightarrow C^{2} N \rightarrow 0
$$

and so on. By splicing, we get an exact sequence, or coresolution:

$$
0 \rightarrow N \stackrel{j}{\rightarrow} D N \rightarrow D C N \rightarrow \cdots \rightarrow D C^{k-1} N \rightarrow D C^{k} N \rightarrow \cdots
$$

When $N$ is a $R \Gamma_{G}$-module of a finite length, which is the case in our situation, this coresolution has a finite length. To check this, we use the definition of $D_{Q}(V)$ in Example 4.8 to get

$$
D_{Q}(V)(K)=\operatorname{Hom}_{R\left[W_{G}(Q)\right]}\left(R\left[(G / K)^{Q}\right], V\right)
$$

for any $R\left[W_{G}(Q)\right]$-module $V$. Therefore $D_{Q}(V)(K)$ is only nonzero for $(Q) \leq(K)$, and at $(Q)=(K)$ the $R\left[W_{G}(K)\right]$-module $D_{Q}(V)(K)$ is isomorphic to $V$, via the isomorphism $W_{G}(Q) \cong W_{G}(K)$ induced by conjugation. This shows that the length of the module $C^{k} N$ is properly smaller than the length of $C^{k-1} N$ for all $k \geq 1$.

We will prove Theorem 5.1 by showing that $H \mapsto \operatorname{Ext}_{R \Gamma_{H}}^{*}(M, N)$ has a cohomological Mackey functor structure which is conjugation invariant. First we describe the Mackey functor structure on $\operatorname{Hom}_{R \Gamma_{?}}(M, N)$. 
Theorem 4.11. For a right $R \Gamma_{G}$-module $M$ and a Mackey functor $N$, let

$$
\operatorname{Hom}_{R \Gamma_{?}}(M, N): \mathcal{D}(G) \rightarrow R-M o d
$$

denote the function defined by $S \mapsto \operatorname{Hom}_{R} \Gamma_{G}\left(M \otimes_{R} R\left[S^{?}\right], N\right)$ for any finite $G$-set $S$. Then $\operatorname{Hom}_{R \Gamma_{?}}(M, N)$ inherits a Mackey functor structure.

Proof. We first define the induction and restriction maps to see that $\operatorname{Hom}_{R \Gamma_{?}}(M, N)$ is a bifunctor. For $f: S \rightarrow T$ a $G$-map, the restriction map

$$
f^{*}: \operatorname{Hom}_{R \Gamma_{G}}\left(M \otimes_{R} R\left[T^{?}\right], N\right) \rightarrow \operatorname{Hom}_{R} \Gamma_{G}\left(M \otimes_{R} R\left[S^{?}\right], N\right)
$$

is the composition with $M \otimes_{R} R\left[S^{?}\right] \stackrel{\text { id } \otimes \tilde{f}}{\longrightarrow} M \otimes_{R} R\left[T^{?}\right]$ where $\tilde{f}$ denotes is the linear extension of the map induced by $f$. Since the functors $R\left[S^{?}\right]$ satisfy axiom $(\mathrm{M} 2)$, so does $\operatorname{Hom}_{R \Gamma_{?}}(M, N)$.

For $f: S \rightarrow T$ a $G$-map, we define the induction map

$$
f_{*}: \operatorname{Hom}_{R \Gamma_{G}}\left(M \otimes_{R} R\left[S^{?}\right], N\right) \rightarrow \operatorname{Hom}_{R \Gamma_{G}}\left(M \otimes_{R} R\left[T^{?}\right], N\right)
$$

in the following way: let $\varphi_{S}: M \otimes_{R} R\left[S^{?}\right] \rightarrow N$ be given. We will describe the homomorphism $\varphi_{T}=f_{*}\left(\varphi_{S}\right)$.

$$
\varphi_{T}(V)(x \otimes \alpha)=F_{*}\left(\varphi_{S}(U)\left(F^{*}(x) \otimes \beta\right)\right)
$$

for $x \in M(V)$ and $\alpha: V \rightarrow T$, where $U, \beta$ and $F$ are given by the pull-back

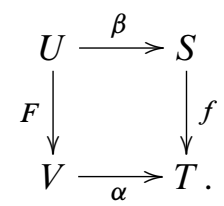

It is easy to check that this formula for $\varphi_{T}$ gives an $R \Gamma_{G}$-homomorphism, using the assumption that $N$ is a Mackey functor.

We need to check axiom $(\mathrm{M} 1)$ for $\operatorname{Hom}_{R \Gamma_{?}}(M, N)$. For a given pull-back square

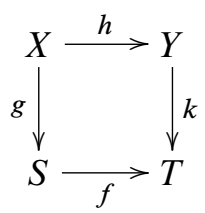

we need to show that $h_{*} \circ g^{*}=k^{*} \circ f_{*}$. Let $\gamma: V \rightarrow Y$ be any $G$-map, and consider the extended pull-back diagram

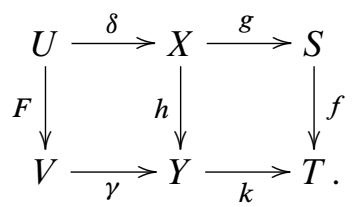


The maps $\alpha=k \circ \gamma$ and $\beta=g \circ \delta$ may be used to compute $f_{*}\left(\varphi_{S}\right)$ as above, and the left-hand square may be used to compute $h_{*}$.

For any element $\varphi_{S}: M \otimes_{R} R\left[S^{?}\right] \rightarrow N$, we have

$$
\begin{aligned}
\left(k^{*} \circ f_{*}\left(\varphi_{S}\right)\right)(V)(x \otimes \gamma) & =\left(f_{*}\left(\varphi_{S}\right) \circ(\mathrm{id} \otimes k)\right)(V)(x \otimes \gamma) \\
& =f_{*}\left(\varphi_{S}\right)(V)(x \otimes(k \circ \gamma)) \\
& =F_{*}\left(\varphi_{S}(U)\left(F^{*}(x) \otimes(g \circ \delta)\right)\right.
\end{aligned}
$$

for any $x \in M(V)$ and $\gamma: V \rightarrow Y$. On the other hand,

$$
\begin{aligned}
\left(h_{*} \circ g^{*}\left(\varphi_{S}\right)\right)(V)(x \otimes \gamma) & =F_{*}\left(\left(g^{*} \varphi_{S}\right)(U)\left(F^{*}(x) \otimes \delta\right)\right) \\
& =F_{*}\left(\varphi_{S}(U)\left(F^{*}(x) \otimes(g \circ \delta)\right)\right.
\end{aligned}
$$

for any $x \in M(V)$ and $\gamma: V \rightarrow Y$, so the formula (M1) is verified.

As an immediate consequence, for any subgroup $H \leq K$ the $G$-map $f: G / H \rightarrow$ $G / K$ induces a restriction map

$$
\operatorname{Res}_{H}^{K}: \operatorname{Hom}_{R \Gamma_{K}}(M, N) \rightarrow \operatorname{Hom}_{R \Gamma_{H}}(M, N)
$$

defined as the composition of the map

$$
f^{*}: \operatorname{Hom}_{R \Gamma_{G}}\left(M \otimes_{R} R\left[G / K^{?}\right], N\right) \rightarrow \operatorname{Hom}_{R \Gamma_{G}}\left(M \otimes_{R} R\left[G / H^{?}\right], N\right)
$$

with the 'Shapiro' isomorphisms:

$$
\operatorname{Hom}_{R \Gamma_{G}}\left(M \otimes_{R} R\left[G / H^{?}\right], N\right) \cong \operatorname{Hom}_{R \Gamma_{H}}(M, N)
$$

and

$$
\operatorname{Hom}_{R \Gamma_{G}}\left(M \otimes_{R} R\left[G / K^{?}\right], N\right) \cong \operatorname{Hom}_{R \Gamma_{K}}(M, N)
$$

given by Corollary 2.12 of [35] and the adjointness property (compare [2], Lemma 2.8.4). Similarly, we have the induction map

$$
\operatorname{Ind}_{H}^{K}: \operatorname{Hom}_{R \Gamma_{H}}(M, N) \rightarrow \operatorname{Hom}_{R \Gamma_{K}}(M, N)
$$

defined by composing the Shapiro isomorphisms with $f_{*}$.

Remark 4.12. Since $\operatorname{Res}_{H}^{G}$ preserves projectives, we see that $P \otimes_{R} R\left[G / H^{?}\right]$ is projective over $R \Gamma_{G}$ whenever $P$ is projective over $R \Gamma_{G}$ (check the categorical lifting property directly or apply Lemma 3.2).

Proposition 4.13. Let $\mathbf{C}$ be a chain complex of right $R \Gamma_{G}$-modules and $N$ be a Mackey functor. Then, the cochain complex

$$
\mathbf{C}^{*}=\operatorname{Hom}_{R \Gamma_{?}}(\mathbf{C}, N)
$$

with the differential $\delta: \operatorname{Hom}_{R \Gamma_{?}}\left(C_{i}, N\right) \rightarrow \operatorname{Hom}_{R \Gamma_{?}}\left(C_{i+1}, N\right)$ given by $\delta(\varphi)=\varphi \circ \partial$ is a cochain complex of Mackey functors. 
Proof. We have seen that each $C^{i}=\operatorname{Hom}_{R \Gamma_{?}}\left(C_{i}, N\right)$ is a Mackey functor by Theorem 4.11. We just need to show that the coboundary maps are Mackey functor maps. Given $f: S \rightarrow T$ we need to show the following diagram commutes:

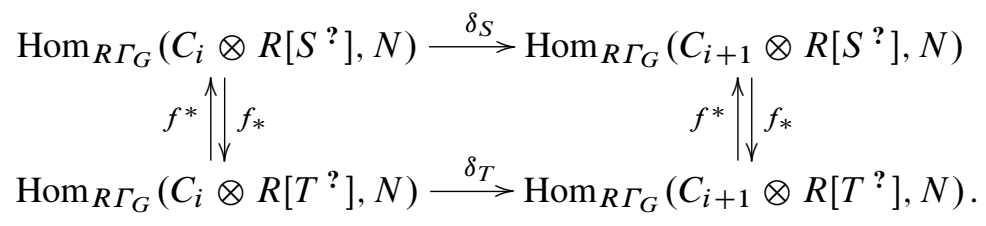

The proof of commutativity for $f^{*}$ is easy. In this case, it follows from the commutativity of the following diagram:

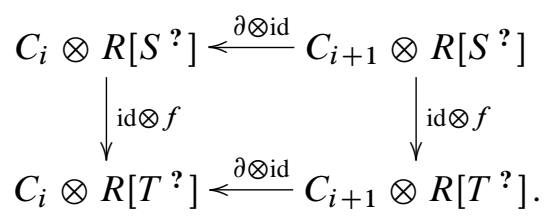

For $f_{*}$ we check the commutativity directly: let $\varphi_{S}: C_{i} \otimes R\left[S^{?}\right] \rightarrow N$ be an $R \Gamma_{G}$-map. For $x \in C_{i+1}(V)$ and $\alpha: V \rightarrow T$, we have

$$
\begin{aligned}
{\left[\left(\delta_{T} \circ f_{*}\right) \varphi_{S}\right](x \otimes \alpha) } & =\left(f_{*} \varphi_{S}\right)(\partial x \otimes \alpha) \\
& =F_{*}\left[\varphi_{S}\left(F^{*}(\partial x) \otimes \beta\right)\right]
\end{aligned}
$$

where

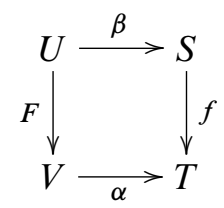

on the other hand,

$$
\begin{aligned}
{\left[\left(f_{*} \circ \delta_{S}\right) \varphi_{S}\right](x \otimes \alpha) } & =F_{*}\left[\left(\delta_{S} \varphi_{S}\right)\left(F^{*}(x) \otimes \beta\right)\right] \\
& =F_{*}\left[\varphi_{S} \circ(\partial \otimes \mathrm{id})\left(F^{*}(x) \otimes \beta\right)\right] \\
& =F_{*}\left[\varphi_{S}\left(\partial F^{*}(x) \otimes \beta\right)\right]
\end{aligned}
$$

since $\partial F^{*}=F^{*} \partial$, we are done.

Corollary 4.14. Let $M$ be an $R \Gamma_{G}$-module and $N$ be a Mackey functor. Then,

$$
\operatorname{Ext}_{R \Gamma_{?}}^{*}(M, N)
$$

has a Mackey functor structure. As a Mackey functor $\operatorname{Ext}_{R \Gamma_{2}}^{*}(M, N)$ is equal to the homology of the cochain complex of Mackey functors $\operatorname{Hom}_{R \Gamma_{?}}(\mathbf{P}, N)$ where $\mathbf{P}$ is a projective resolution of $M$ as an $R \Gamma_{G}$-module. 
Proof. To compute the Ext-groups, note that $S \mapsto \mathbf{P} \otimes_{R} R\left[S^{?}\right]$ is a projective resolution of the module $S \mapsto M \otimes_{R} R\left[S^{?}\right]$, for every finite $G$-set $S$.

Remark 4.15. It follows that a version of the Eckmann-Shapiro isomorphism

$$
\operatorname{Ext}_{R \Gamma_{G}}^{*}\left(M \otimes R\left[G / H^{?}\right], N\right) \cong \operatorname{Ext}_{R \Gamma_{H}}^{*}\left(\operatorname{Res}_{H}^{G} M, \operatorname{Res}_{H}^{G} N\right)
$$

holds for the Ext-groups over the orbit category (compare [2], 2.8.4).

Remark 4.16. If $N$ is a Green module over a Green ring $\mathscr{E}$, then the Mackey functor $\operatorname{Ext}_{R \Gamma_{?}}^{*}(M, N)$ also inherits a Green module structure over $\mathscr{G}$. The basic formula is a pairing

$$
\mathcal{E}(S) \times \operatorname{Hom}_{R \Gamma_{?}}\left(M \otimes_{R} R\left[S^{?}\right], N\right) \rightarrow \operatorname{Hom}_{R \Gamma_{?}}\left(M \otimes_{R} R\left[S^{?}\right], N\right)
$$

induced by the Green module pairing $\mathscr{E} \times N \rightarrow N$. For any $z \in \mathscr{E}(S), x \in M(V)$, and $\alpha: V \rightarrow S$, we define

$$
\left(z \cdot \varphi_{S}\right)(V)(x \otimes \alpha)=\alpha^{*}(z) \cdot \varphi_{S}(V)(x \otimes \alpha)
$$

for any $\varphi_{S}(V): M(V) \otimes_{R} R \operatorname{Mor}(S, V) \rightarrow N(V)$. The check that this pairing gives a Green module structure is left to the reader.

\section{The proof of Theorem $C$}

The main purpose of this section to prove the following theorem.

Theorem 5.1. Let $G$ be a finite group, $R=\mathbb{Z}_{(p)}$, and $\mathscr{F}$ be a family of subgroups in $G$. Suppose $H \leq G$ controls $p$-fusion in $G$. Then,

$$
\operatorname{Res}_{H}^{G}: \operatorname{Ext}_{R \Gamma_{G}}^{n}(M, N) \rightarrow \operatorname{Ext}_{R \Gamma_{H}}^{n}\left(\operatorname{Res}_{H}^{G} M, \operatorname{Res}_{H}^{G} N\right)
$$

is an isomorphism for $n \geqslant 0$, provided that $M$ is an $R \Gamma_{G}$-module and $N$ is a cohomological Mackey functor satisfying the condition that $C_{G}(Q)$ acts trivially on $N(Q)$ and $M(Q)$ for all $p$-subgroups $Q \leq H$, with $Q \in \mathcal{F}$.

Certain Mackey functors (called cohomological) are computable by restriction to the $p$-Sylow subgroups and the conjugation action of $G$ (see [7], Chapter XII, $\$ 10$, [19]).

If $H \leq G$ is a subgroup, and $n \in N_{G}(H)$ then the $G$-map $f: G / H \rightarrow G / H$ defined by $f(e H)=n H$ has an associated conjugation homomorphism $c_{n}(h)=$ $n^{-1} h n \in H$, for all $h \in H$. For an arbitrary $R \Gamma_{G}$-module $M$, the induced maps $f^{*}$ need not be the identity on $M(G / H)$ even if $c_{n}=$ id (e.g. if $n \in C_{G}(H)$ ). 
Definition 5.2. We say a Mackey functor is cohomological (over $\mathcal{F}$ ) if

$$
\operatorname{Ind}_{H}^{K} \operatorname{Res}_{H}^{K}(u)=|K: H| \cdot u
$$

for all $u \in M(K)$, and all $H \leq K$ (for all $K \in \mathcal{F}$ ). An $R \Gamma_{G}$-module $M$ with respect to a family $\mathcal{F}$ is called conjugation invariant if $C_{G}(Q)$ acts trivially on $M(Q)$ for all $Q \in \mathcal{F}$. A Mackey functor is called conjugation invariant if it is conjugation invariant as a functor over the corresponding orbit category.

The following lemma will be used in the proof of Theorem 5.9.

Lemma 5.3. Let $Q \in \mathcal{F}$ and let $V$ be a right $R\left[W_{Q}(Q)\right]$-module. If $\mathcal{F}$ is a family of $p$-subgroups, and $R=\mathbb{F}_{p}$, then $D_{Q}(V): \mathcal{D}(G) \rightarrow R$-Mod is a cohomological Mackey functor over $\mathcal{F}_{\text {. If }} C_{G}(Q)$ acts trivially on $V$, then $D_{Q}(V)$ is conjugation invariant.

Proof. Since all subgroups in $\mathcal{F}$ are $p$-groups, for the first part we only need to show that the composite $\operatorname{Ind}_{H}^{K} \operatorname{Res}_{H}^{K}(u)=p \cdot u$, for $K \in \mathcal{F}$ and $H \leq K$ a normal of index $p$.

Let $f: G / H \rightarrow G / K$ be the $G$-map given by $g H \mapsto g K$. Consider the induced $\operatorname{map} f^{Q}:(G / H)^{Q} \rightarrow(G / K)^{Q}$. Take $t \in(G / K)^{Q}$. If there is no $s \in(G / H)^{Q}$ such that $f(s)=t$, then the transfer is trivially zero. Suppose that there is at least one element $s=g H$ which is fixed by $Q$ and maps to $t=g K$. Let $k_{1}, \ldots, k_{p}$ be coset representatives of $H$ in $K$. Since $k_{i}$ normalizes $H$, the element $g k_{i} H \in(G / H)^{Q}$ for each $i$. Therefore, there are exactly $p$ different $s \in(G / H)^{Q}$ that map to $t$. It follows that $f_{*} \circ f^{*}$ is multiplication by $p$, as required. Since we are working here over the finite field $\mathbb{F}_{p}$, all the composites $f_{*} \circ f^{*}=0$.

We now show that $D_{Q}(V)$ is conjugation invariant if $C_{G}(Q)$ acts trivially on $V$. In other words, we claim that for all $K \in \mathcal{F}$, the centralizer $C_{G}(K)$ acts trivially on $\operatorname{Hom}_{R\left[W_{G}(Q)\right]}\left(R[G / K]^{Q}, V\right)$. Consider the way the action is defined: let $c \in C_{G}(K)$ and $\varphi: R[G / K]^{Q} \rightarrow V$ be an $R\left[W_{G}(Q)\right]$-map. Then $(c \varphi)(g K)=\varphi(g c K)$. On the other hand since $g K \in(G / K)^{Q}$, we have $Q^{g} \leq K$. So, $c$ centralizes $Q^{g}$. This means $g c g^{-1}$ centralizes $Q$ and hence acts trivially on $V$. This gives

$$
\varphi(g c K)=\varphi\left(g c g^{-1} g K\right)=g c g^{-1} \varphi(g K)=\varphi(g K) .
$$

Therefore $(c \varphi)(g K)=\varphi(g K)$ for all $g K$. This shows that $c \in C_{G}(K)$ acts as the identity on $\operatorname{Hom}_{R\left[W_{G}(Q)\right]}\left(R[G / K]^{Q}, V\right)$.

The cohomological and conjugation properties are inherited by the Ext-functors.

Proposition 5.4. Let $M$ and $N$ be $R \Gamma_{G}$-modules relative to some family $\mathcal{F}$.

(i) If $N$ is a cohomological Mackey functor over $\mathcal{F}$, then $\operatorname{Ext}_{R \Gamma_{?}}^{*}(M, N)$ is a cohomological Mackey functor over all subgroups $H \leq G$. 
(ii) If both $M$ and $N$ are conjugation invariant with respect to $\mathcal{F}$, then $\operatorname{Ext}_{R \Gamma_{?}}^{*}(M, N)$ is conjugation invariant with respect to all subgroups $H \leq G$.

Proof. We have seen that for $f: S \rightarrow T$, the induced maps

$$
\operatorname{Hom}_{R} \Gamma_{G}\left(M \otimes R\left[S^{?}\right], N\right) \underset{f^{*}}{\stackrel{f_{*}}{\rightleftarrows}} \operatorname{Hom}_{R \Gamma_{G}}\left(M \otimes R\left[T^{?}\right], N\right)
$$

satisfy the property that

$$
\begin{aligned}
{\left[\left(f_{*} \circ f^{*}\right) \varphi_{T}\right](V)(x \otimes \alpha) } & =F_{*}\left[f^{*}\left(\varphi_{T}\right)(U)\left(F^{*}(x) \otimes \beta\right)\right] \\
& =F_{*}\left[\varphi_{T}(U)\left(F^{*}(x) \otimes(f \circ \beta)\right)\right] \\
& =F_{*}\left[\varphi_{T}(U)\left(F^{*}(x) \otimes(\alpha \circ F)\right)\right] \\
& =\left(F_{*} \circ F^{*}\right)\left[\varphi_{T}(V)(x \otimes \alpha)\right]
\end{aligned}
$$

for all $x \in M(V)$ and $\alpha: V \rightarrow T$. In the last equality we used the invariance of $\varphi_{T}$ with respect to the $G$-map $F: U \rightarrow V$ (our notation comes from the definition of $f_{*}$ above). Hence, if $f: G / H \rightarrow G / K$ and $F_{*} \circ F^{*}$ is multiplication by $|K: H|$ (this follows from a count of double cosets), then $f_{*} \circ f^{*}$ is also multiplication by $|K: H|$.

Let $M$ and $N$ be conjugation invariant right $R \Gamma_{G}$-modules, and let $\mathbf{P}$ be a projective resolution of $M$ over $R \Gamma_{G}$. To show that $\operatorname{Ext}_{R \Gamma_{?}}^{*}(M, N)$ is conjugation invariant, it is enough to show that the chain map induced by the conjugation action on $\operatorname{Hom}_{R \Gamma_{?}}(\mathbf{P}, N)$ is homotopy equivalent to the identity. We remark that the action of an element $c \in C_{G}(H)$ gives an automorphism $J_{c}: \operatorname{Or}_{\mathcal{F}} H \rightarrow \mathrm{Or}_{\mathscr{F}} H$, and induces an $R \Gamma_{H}$-module chain map $\mathbf{P}\left(J_{c}\right): \operatorname{Res}_{H}^{G}(\mathbf{P}) \rightarrow \operatorname{Res}_{H}^{G}(\mathbf{P})$.

If $f: G / H \rightarrow G / H$ is given by $e H \mapsto c H$ where $c \in C_{G}(H)$, then for each degree $i$,

$$
f_{i}^{*}: \operatorname{Hom}_{R \Gamma_{G}}\left(P_{i} \otimes R\left[G / H^{?}\right], N\right) \rightarrow \operatorname{Hom}_{R \Gamma_{G}}\left(P_{i} \otimes R\left[G / H^{?}\right], N\right)
$$

is given by

$$
f_{i}^{*}\left(\varphi_{S}\right)(U)(x \otimes \alpha)=\varphi_{S}(U)(x \otimes f \circ \alpha)
$$

where $S=G / H, x \in P_{i}(U)$, and $\alpha: U \rightarrow G / H$ is a $G$-map. In other words, $f_{i}^{*}=\operatorname{Hom}_{R \Gamma_{G}}\left(\lambda_{i}, \mathrm{id}\right)$, where $\lambda_{i}(x \otimes \alpha)=x \otimes f \circ \alpha$ defines a chain map

$$
\lambda: \mathbf{P} \otimes R\left[G / H^{?}\right] \rightarrow \mathbf{P} \otimes R\left[G / H^{?}\right]
$$

We may assume that $U=G / K$ with $K \in \mathcal{F}$. Let $\alpha(e K)=g H$. The conjugation action of $c \in C_{G}(H)$ on $M(U)$ or $N(U)$ is given by the $G$-map $F: G / K \rightarrow G / K$, where $F(e K)=g c g^{-1} K$ and $f \circ \alpha=\alpha \circ F$. We remark that $z:=g c g^{-1} \in C_{G}(K)$, since $K \subseteq g H g^{-1}$, and that $\mathbf{P}^{*}(F)=\mathbf{P}\left(J_{z}\right)(K)$. Notice that

$$
f_{i}^{*}\left(\varphi_{S}\right)(U)(x \otimes \alpha)=\left(\varphi_{S}(U)\left(x \cdot P_{i}^{*}(F)^{-1} \otimes \alpha\right)\right) \cdot N^{*}(F),
$$


showing that the maps $f_{i}^{*}$ are just given by the natural action maps of $c$ on the domain and range of the Hom. Now observe that

$$
\mathbf{P}\left(J_{z}\right): \operatorname{Res}_{K}^{G}(\mathbf{P}) \rightarrow \operatorname{Res}_{K}^{G}(\mathbf{P})
$$

is a chain map lifting $M\left(J_{z}\right): \operatorname{Res}_{K}^{G}(M) \rightarrow \operatorname{Res}_{K}^{G}(M)$. Since $M$ is conjugation invariant, it follows that $\mathbf{P}\left(J_{z}\right) \simeq$ id by uniqueness (up to chain homotopy) of lifting in projective resolutions. Therefore $\lambda_{1}:=\lambda \circ\left(\mathbf{P}^{*}(F) \otimes\right.$ id $) \simeq \lambda$, and $f^{*} \simeq$ $\operatorname{Hom}\left(\lambda_{1}\right.$, id $)$. But for all $x \in P_{i}(U)$, we have

$\operatorname{Hom}\left(\lambda_{1}, \mathrm{id}\right)\left(\varphi_{S}\right)(U)(x \otimes \alpha)=\varphi_{S}(U)\left(x \cdot P_{i}^{*}(F) \otimes f \circ \alpha\right)=\left(\varphi_{S}(U)(x \otimes \alpha)\right) \cdot N^{*}(F)$,

and hence $f^{*}\left(\varphi_{S}\right) \simeq \varphi_{S}$, by the conjugation invariance of $N$.

Definition 5.5. For any subgroup $H \leq G$, and any $R \Gamma_{G}$-modules $M$ and $N$, an element $\alpha \in \operatorname{Ext}_{R \Gamma_{H}}^{n}(M, N)$ is called stable with respect to $G$ provided that

$$
\operatorname{Res}_{H \cap{ }^{g} H}^{H}(\alpha)=\operatorname{Res}_{H \cap{ }^{g} H}^{g} c_{H}^{g}(\alpha)
$$

for any $g \in G$. The map $c_{H}^{g}$ is the induced map $f_{*}$ where $f: G / H \rightarrow G /{ }^{g} H$ is the $G$-map given by $x H \rightarrow x g^{-1}\left(g H^{-1}\right)$.

Theorem 5.6. Let $R=\mathbb{Z}_{(p)}$ and $G$ be a finite group. For a right $R \Gamma_{G}$-module $M$ and a cohomological Mackey functor $N: \mathscr{D}(G) \rightarrow R$-Mod, the restriction map

$$
\operatorname{Res}_{P}^{G}: \operatorname{Ext}_{R \Gamma_{G}}^{n}(M, N) \rightarrow \operatorname{Ext}_{R \Gamma_{P}}^{n}(M, N)
$$

is an isomorphism for $n \geqslant 0$ onto the stable elements, for any p-Sylow subgroup $P \leq G$.

Proof. By Proposition 5.4 (i), $\operatorname{Ext}_{R \Gamma_{2}}^{n}(M, N)$ is a cohomological Mackey functor. Now the result follows (as in [33], 2.2) from the stable element method of Cartan and Eilenberg [7], Chapter XII, 10.1.

Remark 5.7. Note that since $\operatorname{Ext}_{R \Gamma_{3}}^{*}(M, N)$ is a cohomological Mackey functor, it is a Green module over the trivial module $\underline{R}$, considered as a Green ring by defining $\operatorname{Ind}_{H}^{K}: \underline{R}(G / H) \rightarrow \underline{R}(G / K)$ to be multiplication by $|K: H|$ (see Example 2.9 in [19]). It follows that $\operatorname{Ext}_{R \Gamma_{?}}^{*}(M, N)$ is computable in the sense of Dress in terms of the $p$-Sylow subgroups (see Example 5.10 in [16]).

The proof of Theorem 5.1. Let $R=\mathbb{Z}_{(p)}$ and $G$ be a finite group. Let $H \leq G$ be a subgroup which controls $p$-fusion in $G$. For any cohomological Mackey functor $F$, the restriction map $\operatorname{Res}_{P}^{G}$ maps surjectively to the stable elements in $F(P)$, for 
any $p$-Sylow subgroup $P \leq G$. If $H$ controls $p$-fusion in $G$, and $F$ is conjugation invariant, then all elements in $F(H)$ are stable and

$$
\operatorname{Res}_{H}^{G}: F(G) \stackrel{\approx}{\longrightarrow} F(H)
$$

is an isomorphism. This follows by a standard argument used to prove one direction of Mislin's theorem in group cohomology (see, for example, Symonds [33], Theorem 3.5, or Benson [2], Proposition 3.8.4). We apply Proposition 5.4 and this remark to the cohomological Mackey functor $F=\operatorname{Ext}_{R \Gamma_{?}}^{n}(M, N)$, and the proof is complete.

In the next section we will need a variation of this result.

Definition 5.8. We say the $N$ is an atomic right $R \Gamma_{G}$-module of type $Q \in \mathcal{F}$, if $N=I_{Q}(N(Q))$ where $I_{Q}$ is the inclusion functor introduced in Section 2.

Theorem 5.9. Let $G$ be a finite group, $R=\mathbb{Z}_{(p)}$, and let $\mathcal{F}$ be a family of $p$ subgroups in $G$. Suppose $H \leq G$ controls p-fusion in $G$. Then, for $R \Gamma_{G}$-modules $M$ and $N$,

$$
\operatorname{Res}_{H}^{G}: \operatorname{Ext}_{R \Gamma_{G}}^{n}(M, N) \rightarrow \operatorname{Ext}_{R \Gamma_{H}}^{n}\left(\operatorname{Res}_{H}^{G} M, \operatorname{Res}_{H}^{G} N\right)
$$

is an isomorphism for $n \geqslant 0$, provided that $C_{G}(Q)$ acts trivially on $M(Q)$ and $N(Q)$ for all $Q \in \mathcal{F}$.

Proof. Without loss of generality, we can assume that $N$ is an atomic $R \Gamma_{G}$-module of type $Q$, with trivial $C_{G}(Q)$-action on $N(Q)$. This follows from the 5-lemma (using the filtration of $N$ in [20], 16.8).

Furthermore, we may also assume that $N(Q)$ is $R$-torsion free. To see this, observe that as an $N_{G}(Q) / Q C_{G}(Q)$-module, $N(Q)$ fits into a short exact sequence $0 \rightarrow L \rightarrow F \rightarrow N(Q) \rightarrow 0$, where $F$ is a free $N_{G}(Q) / Q C_{G}(Q)$-module. By taking inflations of these modules, we can consider the sequence as a sequence of $N_{G}(Q) / Q$-modules and apply the functor $I_{Q}$. This shows that $N$ fits into a sequence $0 \rightarrow N^{\prime \prime} \rightarrow N^{\prime} \rightarrow N \rightarrow 0$, where both $N^{\prime}$ and $N^{\prime \prime}$ are conjugation invariant and atomic, with an $R$-torsion free module at $Q$.

Now let $N_{p}=N \otimes \mathbb{F}_{p}=N / p N$. By Lemma 4.10 we have a finite length coresolution

$$
0 \rightarrow N_{p} \rightarrow D N_{p} \rightarrow D C N_{p} \rightarrow \cdots \rightarrow D C^{m} N_{p} \rightarrow 0
$$

for some $m \geq 0$. Since $\mathcal{F}$ is family of $p$-groups, Lemma 5.3 shows that the Mackey functors $D C^{i} N_{p}$ are cohomological over $\mathscr{F}$ and conjugation invariant, for $0 \leq i \leq m$. 
We can apply the functors $\operatorname{Ext}_{R \Gamma_{2}}^{*}(M,-)$ to the coresolution (5.10). By Proposition 5.4, the Mackey functors $\operatorname{Ext}_{R \Gamma_{2}}^{*}\left(M, D C^{i} N_{p}\right)$ are also cohomological and conjugation invariant. Therefore

$$
\operatorname{Res}_{H}^{G}: \operatorname{Ext}_{R \Gamma_{G}}^{*}\left(M, N_{p}\right) \rightarrow \operatorname{Ext}_{R \Gamma_{H}}^{*}\left(M, N_{p}\right)
$$

is an isomorphism by Theorem 5.1 and the 5-lemma (using the coresolution). Furthermore, since $N(Q)$ is $R$-torsion free, we have a short exact sequence

$$
0 \rightarrow N / p^{k-1} \rightarrow N / p^{k} \rightarrow N / p \rightarrow 0,
$$

for every $k \geq 1$, and hence by "dévissage" we conclude that

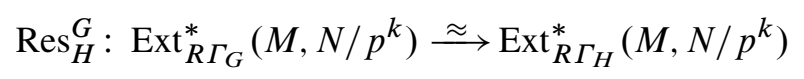

is an isomorphism, for every $k \geq 1$. To finish the proof it is enough to show that

$$
\operatorname{Res}_{H}^{G}: \operatorname{Ext}_{R \Gamma_{G}}^{*}(M, N) \otimes \widehat{\mathbb{Z}}_{p} \rightarrow \operatorname{Ext}_{R \Gamma_{H}}^{*}(M, N) \otimes \widehat{\mathbb{Z}}_{p}
$$

is an isomorphism. However, for $\mathbf{P}$ any projective resolution of $M$ over $R \Gamma_{G}$, the complex

$$
\operatorname{Hom}_{R \Gamma_{G}}\left(\mathbf{P}, N / p^{k}\right)=\operatorname{Hom}_{R \Gamma_{G}}(\mathbf{P}, N) \otimes \mathbb{Z} / p^{k}
$$

is a cochain complex of finitely-generated $R$-modules. By the universal coefficient theorem in cohomology [30], p. 246, we have an exact sequence

$$
\begin{gathered}
0 \rightarrow \operatorname{Ext}_{R \Gamma_{G}}^{n}(M, N) \otimes \mathbb{Z} / p^{k} \rightarrow \operatorname{Ext}_{R \Gamma_{G}}^{n}\left(M, N / p^{k}\right) \\
\rightarrow \operatorname{Tor}_{1}^{R}\left(\operatorname{Ext}_{R \Gamma_{G}}^{n+1}(M, N), \mathbb{Z} / p^{k}\right) \rightarrow 0 .
\end{gathered}
$$

Since $\widehat{\mathbb{Z}}_{p}=\lim _{\longleftarrow} \mathbb{Z} / p^{k}$ and the inverse limit functor is left exact, we obtain an exact sequence

$$
\begin{aligned}
0 \rightarrow \operatorname{Ext}_{R \Gamma_{G}}^{n}(M, N) \otimes \hat{\mathbb{Z}}_{p} \rightarrow \lim _{\longleftarrow} \operatorname{Ext}_{R \Gamma_{G}}^{n}\left(M, N / p^{k}\right) \\
\rightarrow \underset{\leftarrow}{\lim } \operatorname{Tor}_{1}^{R}\left(\operatorname{Ext}_{R \Gamma_{G}}^{n+1}(M, N), \mathbb{Z} / p^{k}\right) .
\end{aligned}
$$

Now we compare this sequence via $\operatorname{Res}_{H}^{G}$ to the corresponding sequence for the subgroup $H$, and use the dévissage isomorphisms (5.11) on the middle term. This shows immediately that $\operatorname{Res}_{H}^{G}$ is injective on the first term, for all $n \geq 0$. Since the functor $\operatorname{Tor}_{1}^{R}$ is left exact, we get $\operatorname{Res}_{H}^{G}$ injective on the third term as well. But now a diagram chase shows that $\operatorname{Res}_{H}^{G}$ is surjective on the first term. 


\section{Chain complexes over orbit categories}

In this section, we prove some theorems about chain complexes over orbit categories. In particular, Proposition 6.8, Proposition 6.4, and Theorem 6.7 will be used in the proof of Theorem A (see Section 9). Most of the results follow from Dold's theory of algebraic Postnikov systems [11].

As before, $G$ denote a finite group and $\mathcal{F}$ denote a family of subgroups of $G$. Throughout this section $\Gamma_{G}=\operatorname{Or}_{\mathscr{F}} G$ and $R$ is a commutative ring. For chain complexes $\mathbf{C}$ and $\mathbf{D}$, the notation $\mathbf{C} \simeq \mathbf{D}$ always means $\mathbf{C}$ is chain homotopy equivalent to $\mathbf{D}$. For chain isomorphism the standard notation is $\mathbf{C} \cong \mathbf{D}$. When we say $\mathbf{C}$ is a projective chain complex, we mean it is a chain complex of projective modules (which also means that it is projective in the category of chain complexes). A chain complex $\mathbf{C}$ is positive if $C_{i}=0$ for $i<0$.

We say that a chain complex $\mathbf{C}$ over $R \Gamma_{G}$ has finite homological dimension (or $\operatorname{hdim} \mathbf{C}$ is finite) if $\mathbf{C}$ is positive, and there exists an integer $n$ such that $H_{i}(\mathbf{C})=0$ for $i>n$. A chain complex $\mathbf{C}$ is finite if $\mathbf{C}$ is positive, and there exists an integer $n$ such that $C_{i}=0$ for $i>n$. We start with a well known observation about chain complexes.

Lemma 6.1. Let $\mathbf{C}$ be a projective chain complex of $R \Gamma_{G}$-modules which has finite homological dimension. Then, $\mathbf{C}$ is homotopy equivalent to a finite projective chain complex if and only if there is an integer $n$ such that

$$
\operatorname{Ext}_{R \Gamma_{G}}^{i}(\mathbf{C}, M)=0 \text { for } i>n,
$$

for all $R \Gamma_{G}$-modules $M$.

Proof. See Cartan-Eilenberg [7], Chapter XVII, 1.4, for chain complexes over rings. A similar argument as in Proposition 4.5 gives the result over the orbit category.

Proposition 6.2. Let $\mathbf{C}$ be a projective chain complex of $\mathbb{Z} \Gamma_{G}$-modules which has a finite homological dimension. Suppose that $\mathbb{Z}_{(p)} \otimes_{\mathbb{Z}} \mathbf{C}$ is chain homotopy equivalent to a finite projective chain complex for all $p|| G \mid$. Then, $\mathbf{C}$ is chain homotopy equivalent to a finite projective complex.

Proof. Let $M$ be an $R \Gamma_{G}$-module. Consider the hypercohomology spectral sequence (see [3], 3.4.3):

$$
E_{2}^{s, t}=\operatorname{Ext}_{\mathbb{Z}}^{s} \Gamma_{G}\left(H_{t}(\mathbf{C}), M\right)
$$

which converges to $\operatorname{Ext}_{\mathbb{Z} \Gamma_{G}}^{*}(\mathbf{C}, M)$. Since $\mathbf{C}$ has finite homological dimension, for all $i>\left(l\left(\Gamma_{G}\right)+\operatorname{hdim} \mathbf{C}\right)$, the group $\operatorname{Ext}_{\mathbb{Z}}^{i}(\mathbf{C}, M)$ is a finite abelian group with exponent dividing a power of $|G|$. Here $l\left(\Gamma_{G}\right)$ is the length of the orbit category, as defined in $\S 2 \mathrm{D}$, and hdim $\mathbf{C}$ denotes the largest integer $n$ such that $H_{n}(\mathbf{C}) \neq 0$. 
In particular, there is an integer $k$, independent from $M$, such that

$$
\operatorname{Ext}_{\mathbb{Z} \Gamma_{G}}^{i}(\mathbf{C}, M) \cong \bigoplus_{p \| G \mid} \operatorname{Ext}_{\mathbb{Z}_{(p)}^{i} \Gamma_{G}}\left(\mathbb{Z}_{(p)} \otimes_{\mathbb{Z}} \mathbf{C}, M_{p}\right)
$$

for all $i>k$. Here $M_{p}=\mathbb{Z}_{(p)} \otimes_{\mathbb{Z}} M$. Now, since $\mathbb{Z}_{(p)} \otimes_{\mathbb{Z}} \mathbf{C}$ is homotopy equivalent to a finite projective complex for all $p|| G \mid$, there is an $n$ such that

$$
\operatorname{Ext}_{\mathbb{Z} \Gamma_{G}}^{i}(\mathbf{C}, M)=0
$$

for all $i>n$ and for all $M$. The result follows from the previous lemma.

A chain complex version of Rim's theorem also holds.

Proposition 6.3. Let $R=\mathbb{Z}_{(p)}$ and $\mathbf{C}$ be a projective chain complex over $R \Gamma_{G}$ with finite homological dimension. Assume that $\mathcal{F}$ is a family of p-subgroups. Then, $\mathbf{C}$ is homotopy equivalent to a finite projective complex if and only if $\operatorname{Res}_{P}^{G} \mathbf{C}$ is homotopy equivalent to a finite projective complex for any $p$-Sylow subgroup $P$ of $G$.

Proof. One direction is clear (and holds without assumption on the family $\mathscr{F}$ ). Conversely, suppose that $\operatorname{Res}_{P}^{G} \mathbf{C}$ is homotopy equivalent to a projective complex with hdim $=l$. Let $n$ be an integer bigger than both $l$ and hdim $\mathbf{C}$. Consider

$$
\cdots \longrightarrow \operatorname{Res}_{P}^{G} C_{n+1} \longrightarrow \operatorname{Res}_{P}^{G} C_{n} \stackrel{\partial_{n}}{\longrightarrow} \operatorname{Res}_{P}^{G} C_{n-1} \longrightarrow \cdots \longrightarrow \operatorname{Res}_{P}^{G} C_{0} \longrightarrow 0 .
$$

We have

$$
\operatorname{Ext}_{R \Gamma_{P}}^{1}\left(\operatorname{Res}_{P}^{G} \operatorname{im}\left(\partial_{n}\right), M\right) \cong \operatorname{Ext}_{R \Gamma_{P}}^{n+1}\left(\operatorname{Res}_{P}^{G} \mathbf{C}, M\right)=0,
$$

for every $R \Gamma_{P}$-module $M$. This gives that $\operatorname{Res}_{P}^{G} \operatorname{im}\left(\partial_{n}\right)$ is projective. By Theorem 3.9, we obtain that $\operatorname{im}\left(\partial_{n}\right)$ has finite projective resolution. Thus, $\mathbf{C}$ is chain homotopy equivalent to a finite projective complex.

We also prove a chain complex version of Theorem 5.9. Recall the definition of conjugation invariant $R \Gamma_{G}$-modules given in (5.2).

Proposition 6.4. Let $G$ be a finite group, and $\mathcal{F}$ be a family of p-subgroups in $G$. Suppose $H \leq G$ controls $p$-fusion in $G$ and $R=\mathbb{Z}_{(p)}$. Let $\mathbf{C}^{H}$ be a positive projective chain complex of $R \Gamma_{H}$-modules such that the homology groups $H_{i}\left(\mathbf{C}^{H}\right)$ are conjugation invariant right $R \Gamma_{H}$-modules, for every $i \geqslant 0$. Then, the following holds:

(i) There exists a positive projective chain complex $\mathbf{C}^{G}$ of $R \Gamma_{G}$-modules such that $\operatorname{Res}_{H}^{G} \mathbf{C}^{G}$ is homotopy equivalent to $\mathbf{C}^{H}$.

(ii) If $\mathbf{C}^{H}$ is homotopy equivalent to a finite projective complex, then $\mathbf{C}^{G}$ is also homotopy equivalent to a finite complex. 
For the proof we will need the theory of algebraic Postnikov systems due to Dold [11], §7. According to this theory, given a positive projective chain complex $\mathbf{C}$, there is a sequence of positive projective chain complexes $\mathbf{C}(i)$ indexed by positive integers such that $f: \mathbf{C} \rightarrow \mathbf{C}(i)$ induces a homology isomorphism for dimensions $\leq i$. Moreover, there is a tower of maps

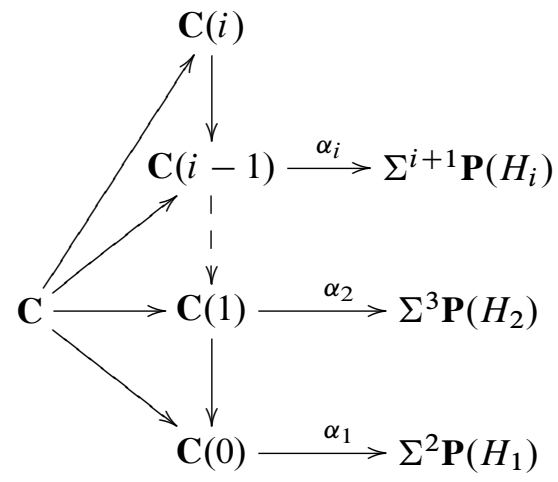

such that $\mathbf{C}(i)=\Sigma^{-1} \mathbf{C}\left(\alpha_{i}\right)$, where $\mathbf{C}\left(\alpha_{i}\right)$ denotes the algebraic mapping cone of $\alpha_{i}$, and $\mathbf{P}\left(H_{i}\right)$ denotes a projective resolution of the homology module $H_{i}$.

Recall that the algebraic mapping cone of a chain map $f: \mathbf{C} \rightarrow \mathbf{D}$ is defined as the chain complex $\mathbf{C}(f)=\mathbf{D} \oplus \Sigma \mathbf{C}$ with boundary map given by $\partial(x, y)=$ $(\partial x+f(x), \partial y)$. Note that $\Sigma^{n}$ is the shift operator for chain complexes which is defined by $\left(\Sigma^{n} \mathbf{C}\right)_{i}=C_{i-n}$ for every integer $n$.

The algebraic Postnikov system has similar properties to the Postnikov system in homotopy theory. The maps $\alpha_{i}: \mathbf{C}(i-1) \rightarrow \Sigma^{i+1} \mathbf{P}\left(H_{i}\right)$ are called $k$-invariants and they are well defined up to chain homotopy equivalence. We can consider the $k$-invariants as classes in $\operatorname{Ext}_{R \Gamma_{G}}^{i+1}\left(\mathbf{C}(i-1), H_{i}\right)$, since there is an isomorphism

$$
\left[\mathbf{C}(i-1), \Sigma^{i+1} \mathbf{P}\left(H_{i}\right)\right] \cong \operatorname{Ext}_{R \Gamma_{G}}^{i+1}\left(\mathbf{C}(i-1), H_{i}\right)
$$

between chain homotopy classes of chain maps and the Ext-groups of chain complexes (see Dold [11] for details). The $k$-invariants $\alpha_{i} \in \operatorname{Ext}_{R \Gamma_{G}}^{i+1}\left(\mathbf{C}(i-1), H_{i}\right)$ are defined inductively and they uniquely specify $\mathbf{C}$ up to chain homotopy equivalence.

We also need a lifting result for $R \Gamma_{H}$-modules.

Lemma 6.5. Let $G$ be a finite group, and $\mathcal{F}$ be a family of $p$-subgroups in $G$. Suppose $H \leq G$ controls p-fusion in $G$. Then the restriction map $M \mapsto \operatorname{Res}_{H}^{G}(M)$ gives a bijection between the isomorphism classes of conjugation invariant right $R \Gamma_{G}$-modules and conjugation invariant right $R \Gamma_{H}$-modules.

Proof. A conjugation invariant right $R \Gamma_{G}$-module $M$ is a functor $\operatorname{Or}_{\mathcal{F}} G \rightarrow R$-Mod which factors through the quotient category $\mathrm{Or}_{\mathscr{F}} G \rightarrow \operatorname{Sub}_{\mathscr{F}} G$. Here $\operatorname{Sub}_{\mathscr{F}} G$ has objects $K \in \mathscr{F}$ and morphisms $\operatorname{Mor}_{\operatorname{Sub}_{\mathscr{F}} G}(K, L)=\operatorname{Mor}_{\text {Or }_{\mathscr{F}} G}(G / K, G / L) / C_{G}(K)$, 
where an element $c \in C_{G}(K)$ acts on a $G$-map defined by $f(e K)=g L$ via the composition $e K \mapsto c g L$ (see p. 206 in [21]).

Consider the functor $F:$ Or $_{\mathcal{F}} H \rightarrow \mathrm{Or}_{\mathcal{F}} G$ given on objects by $H / K \mapsto G / K$ (see Section 3), and on morphisms by induced maps. First note that every object of $\operatorname{Sub}_{\mathcal{F}} G$ is isomorphic to an object of $\operatorname{Sub}_{\mathcal{F}} H$, since every $p$-subgroup of $G$ is conjugate to a subgroup of $H$. In addition, $F$ induces a bijection of morphism sets

$$
\operatorname{Mor}_{\operatorname{Sub}_{\mathscr{F}} H}(K, L) \rightarrow \operatorname{Mor}_{\operatorname{Sub}_{\mathscr{F}} G}(K, L)
$$

since $H$ controls $p$-fusion in $G$. Suppose that $F\left(f_{1}\right) \approx F\left(f_{2}\right)$, where $f_{1}(e K)=h_{1} L$ and $f_{2}(e K)=h_{2} L$, for some $h_{1}, h_{2} \in H$. By assumption, there exists $c \in C_{G}(K)$ such that $c h_{2} L=h_{1} L$, or $h_{1}^{-1} c h_{2} \in L \leq H$. But this implies $c \in C_{H}(K)$ so $f_{1} \approx f_{2}$ and $F$ is injective on morphisms. Given $f: G / K \rightarrow G / L$ with $K \leq H$, $f(e K)=g L$ and $g^{-1} K g \subseteq L \leq H$, we have $g=c h$ for some $c \in C_{G}(K)$ and $h \in H$, because $H$ controls $p$-fusion in $G$. Hence $f \approx F\left(f_{1}\right)$, where $f_{1}(e K)=h L$ and $F$ is surjective on morphisms.

Therefore the functor $F: \operatorname{Or}_{\mathcal{F}} H \rightarrow \mathrm{Or}_{\mathcal{F}} G$ induces an equivalence of categories

$$
\bar{F}: \operatorname{Sub}_{\mathscr{F}} H \approx \operatorname{Sub}_{\mathscr{F}} G
$$

by [24], IV.4, Theorem 1, p. 91.

Proof of Proposition 6.4. Part (ii) follows from Proposition 6.3, so it is enough to prove the existence of $\mathbf{C}^{G}$. By Lemma 6.5, for each $i \geqslant 0$ there exists a conjugation invariant right $R \Gamma_{G}$-module $H_{i}^{G}$ such that $\operatorname{Res}_{H}^{G}\left(H_{i}^{G}\right)=H_{i}\left(\mathbf{C}^{H}\right)$.

Consider the Postnikov tower for $\mathbf{C}^{H}$. Since $\mathbf{C}^{H}(0)=\mathbf{P}\left(H_{0}\left(\mathbf{C}^{H}\right)\right)$ there is a complex $\mathbf{C}^{G}(0)$ such that $\operatorname{Res}_{H}^{G} \mathbf{C}^{G}(0) \simeq \mathbf{C}^{H}(0)$. In this case, the complex $\mathbf{C}^{G}(0)$ can be taken as a projective resolution of $H_{0}^{G}$. Now, we will show that such a lifting exists for $\mathbf{C}^{H}(i)$ for all $i$. For this we prove a slightly stronger statement so that we can carry out an induction. We claim that the following holds for all $n \geqslant 0$.

(i) $\mathbf{C}^{H}(n)$ lifts to a chain complex $\mathbf{C}^{G}(n)$

(ii) The restriction map

$$
\operatorname{Res}_{H}^{G}: \operatorname{Ext}_{R \Gamma_{G}}^{*}\left(\mathbf{C}^{G}(n), N\right) \rightarrow \operatorname{Ext}_{R \Gamma_{H}}^{*}\left(\mathbf{C}^{H}(n), \operatorname{Res}_{H}^{G} N\right)
$$

is an isomorphism for all $* \geqslant 0$ and for every $R \Gamma_{G}$-module $N$ which is conjugation invariant.

We have already shown that $\mathbf{C}^{H}(0)$ lifts to $\mathbf{C}^{G}(0)$. For the second property, first observe that $\mathbf{C}^{G}(0)$ is chain homotopy equivalent to a chain complex with single module $H_{0}^{G}$ and similarly, $\mathbf{C}^{H}(0) \simeq H_{0}^{H}$. So, we need to show that

$$
\operatorname{Res}_{H}^{G}: \operatorname{Ext}_{R \Gamma_{G}}^{*}\left(H_{0}^{G}, H_{1}^{G}\right) \rightarrow \operatorname{Ext}_{R \Gamma_{H}}^{*}\left(H_{0}^{H}, H_{1}^{H}\right)
$$


is an isomorphism. This follows from Theorem 5.9, because of our assumption on homology groups.

Now, assume that both (i) and (ii) hold for $n=i-1$. Then, take

$$
\alpha_{i}^{G} \in \operatorname{Ext}_{R \Gamma_{G}}^{i+1}\left(\mathbf{C}^{G}(i-1), H_{i}^{G}\right)
$$

which corresponds to the class $\alpha_{i}^{H} \in \operatorname{Ext}_{R \Gamma_{H}}^{i+1}\left(\mathbf{C}^{H}(i-1), H_{i}^{H}\right)$ under the isomorphism given in (ii). Let $\mathbf{C}^{G}(i)=\Sigma^{-1} \mathbf{C}\left(\alpha_{i}^{G}\right)$. Then, we have a short exact sequence of chain complexes

$$
0 \longrightarrow \mathbf{C}^{G}(i) \longrightarrow \mathbf{C}^{G}(i-1) \stackrel{\alpha_{i}^{G}}{\longrightarrow} \Sigma^{i+1} \mathbf{P}\left(H_{i}^{G}\right) \longrightarrow 0 .
$$

Since $\operatorname{Res}_{H}^{G} \alpha_{i}^{G}=\alpha_{i}^{H}$, we have $\operatorname{Res}_{H}^{G} \mathbf{C}^{G}(i) \simeq \mathbf{C}^{H}(i)$. Now, we will show that (ii) holds for $\mathbf{C}^{G}(i)$. By the 5-lemma, it is enough to show that

$$
\operatorname{Res}_{H}^{G}: \operatorname{Ext}_{R \Gamma_{G}}^{*}\left(\Sigma^{i+1} \mathbf{P}\left(H_{i}^{G}\right), N\right) \rightarrow \operatorname{Ext}_{R \Gamma_{H}}^{*}\left(\Sigma^{i+1} \mathbf{P}\left(H_{i}^{H}\right), N\right)
$$

is an isomorphism for all $* \geqslant 0$, and for every $R \Gamma_{G}$-module $N$ which is conjugation invariant. But, this follows from Theorem 5.9.

Now, we prove one of the main results of this section which allows us to glue $p$-local chain complexes. We first give a definition.

Definition 6.6. Let $\mathbf{C}$ be a chain complex over $R \Gamma_{G}$. We say that $\mathbf{C}$ has homology gaps of length $n$, if $H_{i+k}(\mathbf{C})=0$ for $0<k<n$, whenever $H_{i}(\mathbf{C}) \neq 0$.

Theorem 6.7. Let $G$ be a finite group of order $m$. For each prime $p \mid m$, let $\mathbf{C}^{(p)}$ be a positive projective chain complex of $\mathbb{Z}_{(p)} \Gamma_{G}$-modules. Suppose that

(i) $\mathbf{C}^{(p)}$ has homology gaps of length $>l\left(\Gamma_{G}\right)$, for all $p \mid m$,

(ii) there exists a graded $\mathbb{Z} \Gamma_{G}$-module $\mathbf{H}$ such that $H_{i}\left(\mathbf{C}^{(p)}\right) \cong \mathbb{Z}_{(p)} \otimes_{\mathbb{Z}} \mathbf{H}_{i}$ for all $i \geq 0$, and for all $p \mid m$.

Then, there is a projective chain complex $\mathbf{C}$ of $\mathbb{Z} \Gamma_{G}$-modules such that $\mathbb{Z}_{(p)} \otimes_{\mathbb{Z}} \mathbf{C} \simeq$ $\mathbf{C}^{(p)}$, for each prime $p \mid m$, and $H_{i}(\mathbf{C})=\mathbf{H}_{i}$ for $i \geq 0$.

Proof. We will construct $\mathbf{C}$ inductively. The case $i=0$ is trivial, because in this case we can take $\mathbf{C}(0)=\mathbf{P}\left(\mathbf{H}_{0}\right)$. Assume now that $\mathbf{C}(i-1)$ has been constructed in such a way that $\mathbb{Z}_{(p)} \otimes_{\mathbb{Z}} \mathbf{C}(i-1) \simeq \mathbf{C}_{i-1}^{(p)}$ for all $p \mid m$. If $\mathbf{H}_{i}=0$, then we can take $\mathbf{C}(i)=\mathbf{C}(i-1)$ and it will satisfy the condition that $\mathbb{Z}_{(p)} \otimes_{\mathbb{Z}} \mathbf{C}(i) \simeq \mathbf{C}_{i}^{(p)}$. So, assume $\mathbf{H}_{i}$ is nonzero. If $i+1>\left(l\left(\Gamma_{G}\right)+\operatorname{hdim} \mathbf{C}(i-1)\right)$, then we have

$$
\begin{aligned}
\operatorname{Ext}_{\mathbb{Z} \Gamma_{G}}^{i+1}\left(\mathbf{C}(i-1), \mathbf{P}\left(\mathbf{H}_{i}\right)\right) & \cong \bigoplus_{p \mid m} \operatorname{Ext}_{\mathbb{Z}_{(p)}^{i+1} \Gamma_{G}}\left(\mathbb{Z}_{(p)} \otimes_{\mathbb{Z}} \mathbf{C}(i-1), \mathbf{H}_{i}^{(p)}\right) \\
& \cong \bigoplus_{p \mid m} \operatorname{Ext}_{\mathbb{Z}_{(p)}^{i+1} \Gamma_{G}}\left(\mathbf{C}^{(p)}(i-1), \mathbf{H}_{i}^{(p)}\right)
\end{aligned}
$$


where $\mathbf{H}_{i}^{(p)}=\mathbb{Z}_{(p)} \otimes_{\mathbb{Z}} \mathbf{H}_{i}$. Note that the above condition on $(i+1)$ is satisfied since the distance between nonzero homology groups of $\mathbf{C}^{(p)}$ is bigger than $l\left(\Gamma_{G}\right)$. Choose $\alpha_{i} \in \operatorname{Ext}_{\mathbb{Z} \Gamma_{G}}^{i+1}\left(\mathbf{C}(i-1), \mathbf{P}\left(\mathbf{H}_{i}\right)\right)$ so that under the $p$-localization map, $\alpha_{i}$ is mapped to the $i$-th $k$-invariant $\alpha_{i}^{(p)}$ of the $p$-local complex $\mathbf{C}^{(p)}$, for every $p \mid m$. Let $\mathbf{C}(i)=\Sigma^{-1} \mathbf{C}\left(\alpha_{i}\right)$. For each prime $p \mid m$, we have a diagram of the form

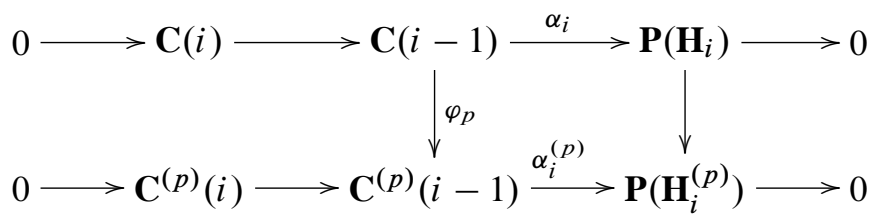

where the vertical map $\varphi_{p}$ is given by the composition

$$
\varphi_{p}: \mathbf{C}(i-1) \rightarrow \mathbb{Z}_{(p)} \otimes_{\mathbb{Z}} \mathbf{C}(i-1) \cong \mathbf{C}^{(p)}(i-1) .
$$

The first map in the above composition is induced by the usual inclusion of integers into $p$-local integers. From this diagram, it is clear that there is a map $\mathbf{C}(i) \rightarrow \mathbf{C}^{(p)}(i)$ which induces an isomorphism on homology when it is localized at $p$. Thus, it gives a chain homotopy equivalence $\mathbb{Z}_{(p)} \otimes_{\mathbb{Z}} \mathbf{C}(i) \simeq \mathbf{C}^{(p)}(i)$, for $p \mid m$. This completes the proof.

We conclude this section with a technique (used in the proof of Theorem A) for modifying the homology of a given (finite, projective) chain complex $\mathbf{C}$ over the orbit category. A projective resolution $\mathbf{P} \rightarrow M$ has length $\leqslant \ell$, provided that $P_{i}=0$ for $i>\ell$.

Proposition 6.8. Let $\Gamma$ be an EI-category. Let $\varphi: \mathbf{H}_{k} \rightarrow \mathbf{H}_{k}^{\prime}$ be an $R \Gamma$-module homomorphism, where $\mathbf{H}_{k}=H_{k}(\mathbf{C})$. Suppose that both kernel and cokernel of $\varphi$ admit finite projective resolutions of length $\leqslant \ell$, and that $\mathbf{H}_{k+j}=0$ for $1 \leqslant j<\ell$. Then there is a $R \Gamma$-chain complex $\mathbf{C}^{\prime}$ such that $H_{i}\left(\mathbf{C}^{\prime}\right)=H_{i}(\mathbf{C})$, for $i \neq k$, and $H_{k}\left(\mathbf{C}^{\prime}\right)=\mathbf{H}_{k}^{\prime}$.

Proof. First suppose that $\varphi$ is surjective. Let

$$
0 \rightarrow P_{k+\ell} \rightarrow \cdots \rightarrow P_{k} \rightarrow \operatorname{ker} \varphi \rightarrow 0
$$

be a projective resolution for $\operatorname{ker} \varphi$. Since $\mathbf{C}$ is exact in the range $[k+1, k+\ell$ ), we have a chain map

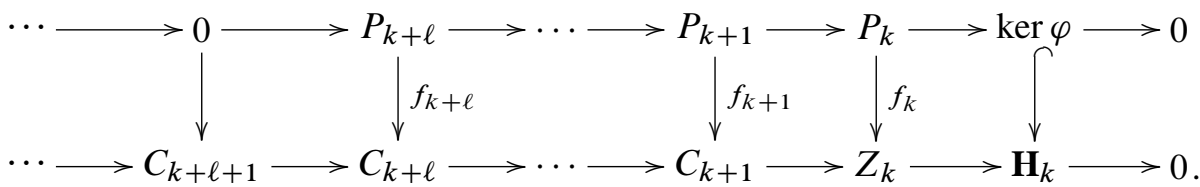


This gives a chain map $f: \mathbf{P} \rightarrow \mathbf{C}$, where $f_{k}: P_{k} \rightarrow C_{k}$ is the composition of $f_{k}$ with the inclusion $Z_{k} \subset C_{k}$. Let $\mathbf{C}^{\prime}=\mathbf{C}(f)$ denote the mapping cone of $f$. The induced map

$$
\operatorname{ker} \varphi=H_{k}(\mathbf{P}) \rightarrow H_{k}(\mathbf{C})=\mathbf{H}_{k}
$$

on homology is given by the inclusion, and hence $H_{k}\left(\mathbf{C}^{\prime}\right)=\mathbf{H}_{k}^{\prime}$, with $H_{i}\left(\mathbf{C}^{\prime}\right)=$ $H_{i}(\mathbf{C})$ for $i \neq k$.

Now suppose that $\varphi$ is an injective map, so that

$$
0 \rightarrow \mathbf{H}_{k} \stackrel{\varphi}{\rightarrow} \mathbf{H}_{k}^{\prime} \rightarrow \operatorname{coker} \varphi \rightarrow 0
$$

is exact. Let $\epsilon: \mathbf{P} \rightarrow \operatorname{coker} \varphi$ be a projective resolution of $\operatorname{coker} \varphi$ of length $\leqslant \ell$, indexed so that $\epsilon: P_{k} \rightarrow \operatorname{coker} \varphi \rightarrow 0$. We form the pull-back

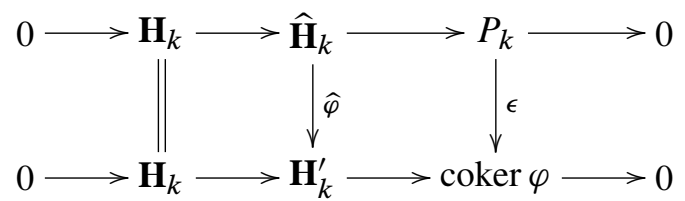

of the sequence (6.9) by $\epsilon$, and note that $\hat{\mathbf{H}}_{k} \cong \mathbf{H}_{k} \oplus P_{k}$. The chain complex

$$
\cdots \rightarrow C_{k+1} \rightarrow C_{k} \oplus P_{k} \rightarrow C_{k-1} \rightarrow \cdots \rightarrow C_{0} \rightarrow 0
$$

has homology $\hat{\mathbf{H}}_{k}$ at $i=k$, and $\hat{\varphi}: \widehat{\mathbf{H}}_{k} \rightarrow \mathbf{H}_{k}^{\prime}$ is surjective. By the pull-back diagram,

$$
\operatorname{ker} \hat{\varphi} \cong \operatorname{ker}\left(\epsilon: P_{k} \rightarrow \operatorname{coker} \varphi\right) \text {. }
$$

Since $\operatorname{coker} \varphi$ has a projective resolution of length $\leq \ell$, it follows that ker $\hat{\varphi}$ has a projective resolution of length $<\ell$. Hence the assumptions needed for the surjective case hold for $\hat{\varphi}: \hat{\mathbf{H}}_{k} \rightarrow \mathbf{H}_{k}^{\prime}$, and we are done by the argument above.

The general case is done by expressing the $\operatorname{map} \varphi: \mathbf{H}_{k} \rightarrow \mathbf{H}_{k}^{\prime}$ as the composition of a surjection and an injection.

\section{The finiteness obstruction}

Let $G$ be a finite group and $\mathscr{F}$ be a family of subgroups of $G$. The main result of this section is Theorem 7.6: given a finite projective chain complex $\mathbf{C}$ of $\mathbb{Z} \Gamma_{G}$-modules, for $\Gamma_{G}=\operatorname{Or}_{\mathcal{F}} G$, we can obtain a finite free complex by taking join tensor of $\mathbf{C}$ with itself sufficiently many times. This result is an adaptation to the orbit category of the fundamental work of Swan [32]. We first introduce some definitions, based on the material in Lück [20], §10-11.

Let $\Gamma$ be an EI-category. We denote by $K_{0}(\mathbb{Z} \Gamma)$ the Grothendieck ring of isomorphism classes of projective $\mathbb{Z} \Gamma$-modules and $K_{0}(\mathbb{Z} \Gamma$, free $)$ denote the Grothendieck 
ring of isomorphism classes of free $\mathbb{Z} \Gamma$-modules (under direct sum $M \oplus N$ and tensor product $\left.M \otimes_{\mathbb{Z}} N\right)$. We have an exact sequence of abelian groups

$$
0 \rightarrow K_{0}(\mathbb{Z} \Gamma, \text { free }) \rightarrow K_{0}(\mathbb{Z} \Gamma) \stackrel{q}{\rightarrow} \widetilde{K}_{0}(\mathbb{Z} \Gamma) \rightarrow 0
$$

defining the quotient group $\tilde{K}_{0}(\mathbb{Z} \Gamma)$.

Note that $K_{0}(\mathbb{Z} \Gamma$, free) is a subring, but not an ideal in general. This is because the tensor product of a free module with a projective module is not free in $\mathbb{Z} \Gamma$. For example, if $P$ is a projective module which is not free, then $P \otimes \underline{\mathbb{Z}} \cong P$ is not a free $\mathbb{Z} \Gamma_{G}$-module although $\underline{\mathbb{Z}}$ is free when $G \in \mathcal{F}$.

Given a finite projective chain complex of $\mathbb{Z} \Gamma$-modules

$$
\mathrm{C}: 0 \rightarrow C_{n} \rightarrow C_{n-1} \rightarrow \cdots \rightarrow C_{1} \rightarrow C_{0} \rightarrow 0
$$

we define

$$
\sigma(\mathbf{C})=\sum_{i=0}^{n}(-1)^{i}\left[C_{i}\right] \in K_{0}(\mathbb{Z} \Gamma)
$$

and

$$
\tilde{\sigma}(\mathbf{C})=q(\sigma(\mathbf{C})) \in \tilde{K}_{0}(\mathbb{Z} \Gamma) .
$$

The class $\tilde{\sigma}(\mathbf{C})$ is called the finiteness obstruction since it is the only obstruction for $\mathbf{C}$ to be chain homotopy equivalent to a finite free chain complex.

From now on, we assume that all the chain complexes are positive and projective. As always, we assume all modules are finitely generated.

The following are standard results which show that $\tilde{\sigma}(\mathbf{C})$ is an invariant, and that it is an obstruction for finiteness.

Lemma 7.1. If $\mathbf{C}$ and $\mathbf{D}$ are chain homotopy equivalent, then $\sigma(\mathbf{C})=\sigma(\mathbf{D})$.

Proof. See [20], 11.2.

Lemma 7.2. Let $\mathbf{C}$ and $\mathbf{D}$ be finite chain complexes of projective $\mathbb{Z} \Gamma$-modules. Then, $\sigma\left(\mathbf{C} \otimes_{\mathbb{Z}} \mathbf{D}\right)=\sigma(\mathbf{C}) \cdot \sigma(\mathbf{D})$.

Proof. See [20], 11.18, and the sharper result in [20], 11.24.

Lemma 7.3. Let $\mathbf{C}$ be a finite chain complex with $\tilde{\sigma}(\mathbf{C})=0$. Then $\mathbf{C}$ is chain homotopy equivalent to a finite chain complex of free $\mathbb{Z} \Gamma$-modules.

Proof. See Swan [32], Proposition 5.1.

Given two chain complexes of $R \Gamma$-modules $\mathbf{C}$ and $\mathbf{D}$, consider the corresponding augmented complexes

$$
\widetilde{\mathbf{C}}: \cdots \rightarrow C_{2} \rightarrow C_{1} \rightarrow C_{0} \rightarrow \underline{R} \rightarrow 0
$$


and

$$
\widetilde{\mathbf{D}}: \cdots \rightarrow D_{2} \rightarrow D_{1} \rightarrow D_{0} \rightarrow \underline{R} \rightarrow 0 .
$$

Taking their tensor product, we obtain a complex of the form

$$
\widetilde{\mathbf{C}} \otimes_{R} \widetilde{\mathbf{D}}: \cdots \rightarrow C_{1} \oplus D_{1} \oplus C_{0} \otimes D_{0} \rightarrow C_{0} \oplus D_{0} \rightarrow \underline{R} \rightarrow 0 .
$$

Definition 7.4. We define the join tensor, denoted $\mathbf{C} * \mathbf{D}$, of two positive augmented chain complexes $C$ and $D$ by the formula

$$
\widetilde{\mathbf{C} * \mathbf{D}}=\Sigma\left(\widetilde{\mathbf{C}} \otimes_{R} \widetilde{\mathbf{D}}\right),
$$

where $\Sigma$ denote the suspension of a chain complex defined by $(\Sigma \mathbf{C})_{i}=C_{i-1}$ for all $i$.

Lemma 7.5. Let $\mathbf{C}$ and $\mathbf{D}$ be finite chain complexes of projective $\mathbb{Z} \Gamma$-modules. Then, $\sigma(\mathbf{C} * \mathbf{D})=\sigma(\mathbf{C})+\sigma(\mathbf{D})-\sigma(\mathbf{C}) \cdot \sigma(\mathbf{D})$.

Proof. Note that $(\mathbf{C} * \mathbf{D})_{k}=C_{k} \oplus D_{k} \oplus \bigoplus_{i+j=k-1} C_{i} \otimes_{\mathbb{Z}} D_{j}$, for each $k \geq 0$. Therefore,

$$
\sigma(\mathbf{C} * \mathbf{D})=\sum_{k}(-1)^{k}\left[C_{k}\right]+\sum_{k}(-1)^{k}\left[D_{k}\right]-\sum_{i+j=k-1}(-1)^{k}\left[C_{i} \otimes D_{j}\right]
$$

and the result follows.

We often express the above formula by writing

$$
(1-\sigma(\mathbf{C} * \mathbf{D}))=(1-\sigma(\mathbf{C}))(1-\sigma(\mathbf{D})) .
$$

Whenever it is written in this way, one should understand it as a formal expression of the formula given in Lemma 7.5. The main theorem of this section is the following:

Theorem 7.6. Let $\Gamma_{G}=\mathrm{Or}_{\mathcal{F}} G$ where $G$ is a finite group and $\mathcal{F}$ is a family of subgroups in G. Given a finite chain complex $\mathbf{C}$ of projective $\mathbb{Z} \Gamma_{G}$-modules, there exists an integer $n$ such that $n$-fold join tensor $*_{n} \mathbf{C}$ of the complex $\mathbf{C}$ is chain equivalent to a finite complex of free $\mathbb{Z} \Gamma_{G}$-modules.

We need to show that the finiteness obstruction $\widetilde{\sigma}\left(*_{n} \mathbf{C}\right)$ vanishes for some $n$. In the proof we will use a result by Oliver and Segev [26].

Proposition 7.7. Let $G$ be a finite group and let $P$ and $P^{\prime}$ be any two finitely generated projective $\mathbb{Z} G$-modules. Then, $P \otimes_{\mathbb{Z}} P^{\prime}$ is stably free as a $\mathbb{Z} G$-module.

Proof. See [26], Proposition C.3. 
We also need the following splitting theorem for $K_{0}(\mathbb{Z} \Gamma)$.

Theorem 7.8. Let $\Gamma$ be an EI category. Then, the map

$$
K_{0}(S): K_{0}(\mathbb{Z} \Gamma) \rightarrow \bigoplus_{x \in \operatorname{Iso}(\Gamma)} K_{0}(\mathbb{Z}[x]),
$$

defined by $[P] \rightarrow\left[S_{x}(P)\right]$ on each $x \in \operatorname{Iso}(\Gamma)$, is an isomorphism. The same holds when $K_{0}$ is replaced by $\widetilde{K}_{0}$.

Proof. See Lück [10], Proposition 11.29.

As a consequence of this theorem, if $\Gamma$ is finite then $\widetilde{K}_{0}(\mathbb{Z} \Gamma)$ is finite: in this case $\Gamma$ has finitely many isomorphism classes of objects $x \in \mathrm{Ob}(\Gamma)$, and $\operatorname{Aut}[x]$ is a finite group (apply Swan [31]), Proposition 9.1. In particular, if $\Gamma=\operatorname{Or}_{\mathcal{F}} G$, then the group $\widetilde{K}_{0}(\Gamma)$ is finite.

From now on we assume $\Gamma_{G}=\operatorname{Or}_{\mathscr{F}} G$ for some finite group $G$, relative to some family $\mathcal{F}$. The splitting theorem above can also be used to give a filtration of $\widetilde{K}_{0}\left(\Gamma_{G}\right)$. Recall that every projective $\mathbb{Z} \Gamma_{G}$-module is of the form

$$
P \cong \bigoplus_{H \in T} E_{H} S_{H} P
$$

where $T$ is a set of representatives of conjugacy classes of elements in $\mathcal{F}$. So, another way to express the above splitting theorem is to write

$$
K_{0}\left(\mathbb{Z} \Gamma_{G}\right) \cong \bigoplus_{H \in T} K_{0}\left(\mathbb{Z} \Gamma_{G}\right)_{H}
$$

where $K_{0}\left(\mathbb{Z} \Gamma_{G}\right)_{H}=\left\{[P] \mid E_{H} S_{H} P \cong P\right\}$. Note that this is only a splitting as abelian groups, but using this we can give a filtration for the ring structure of $K_{0}\left(\mathbb{Z} \Gamma_{G}\right)$. Let

$$
\emptyset=T_{0} \subseteq T_{1} \subseteq \cdots \subseteq T_{m}=T
$$

be a filtration of $T$ such that if $H \in T_{i}$ and $K \in T_{j}$ and ${ }^{g} H \leq K$ for some $g \in G$, then $i \leqslant j$. This gives a filtration

$$
0=K_{0}\left(\mathbb{Z} \Gamma_{G}\right)_{0} \subseteq K_{0}\left(\mathbb{Z} \Gamma_{G}\right)_{1} \subseteq \cdots \subseteq K_{0}\left(\mathbb{Z} \Gamma_{G}\right)_{m}=K_{0}\left(\mathbb{Z} \Gamma_{G}\right)
$$

where

$$
K_{0}\left(\mathbb{Z} \Gamma_{G}\right)_{i}=\left\{[P] \mid P=\bigoplus_{H \in T_{i}} E_{H} S_{H} P\right\} .
$$

Lemma 7.9. Let $V$ be a $\mathbb{Z}\left[N_{G}(H) / H\right]$-module and $W$ be a $\mathbb{Z}\left[N_{G}(K) / K\right]$-module. Then,

$$
E_{H} V \otimes_{\mathbb{Z}} E_{K} W \cong \bigoplus_{H g K \in H \backslash G / K} E_{H \cap{ }^{g} K}\left(\operatorname{Res}_{H \cap{ }^{g} K} E_{H} V \otimes_{\mathbb{Z}} \operatorname{Res}_{H \cap{ }^{g}} E_{K} W\right) .
$$


Proof. Applying the definition, we get

$$
E_{H} V \otimes_{\mathbb{Z}} E_{K} W=(V \otimes W) \otimes_{\mathbb{Z}[\operatorname{Aut}(G / H) \times \operatorname{Aut}(G / K)]} \mathbb{Z} \operatorname{Map}_{G}(?, G / H \times G / K)
$$

where $\operatorname{Map}_{G}(X, Y)$ denotes the set $G$-sets from $X$ to $Y$ (see [10], 11.30, for a similar computation). Since

$$
G / H \times G / K=\coprod_{H g K \in H \backslash G / K} G /\left(H \cap{ }^{g} K\right),
$$

the module $E_{H} V \otimes_{R} E_{K} W$ decomposes as

$$
\bigoplus_{H g K \in H \backslash G / K} E_{H \cap{ }^{g} K} U_{H \cap{ }^{g} K}
$$

where $U_{H \cap{ }^{g} K}$ are $N_{G}\left(H \cap{ }^{g} K\right) /\left(H \cap{ }^{g} K\right)$-modules. Applying $S_{H \cap{ }^{g} K}$, we find

$$
\begin{aligned}
U_{H \cap{ }^{g} K}=S_{H \cap{ }^{g} K}\left(E_{H} V \otimes_{\mathbb{Z}} E_{K} W\right) & =\operatorname{Res}_{H \cap{ }^{g} K}\left(E_{H} V \otimes_{\mathbb{Z}} E_{K} W\right) \\
& =\operatorname{Res}_{H \cap{ }^{g} K} E_{H} V \otimes_{\mathbb{Z}} \operatorname{Res}_{H \cap{ }^{g} K} E_{K} W .
\end{aligned}
$$

Lemma 7.10. $K_{0}\left(\mathbb{Z} \Gamma_{G}\right)_{i}$ is an ideal of $K_{0}\left(\mathbb{Z} \Gamma_{G}\right)$.

Proof. For $E_{H} S_{H} P$ and $E_{K} S_{K} Q$, we have

$$
E_{H} S_{H} P \otimes_{\mathbb{Z}} E_{K} S_{K} Q=\bigoplus_{L} E_{L} V_{L}
$$

where $L=H \cap{ }^{g} K$ for some $g \in G$. So, if $H \in T_{i}, K \in T_{j}$, and $L \in T_{k}$, then $k \leqslant i, j$.

Now, Theorem 7.6 follows by induction from the following proposition.

Proposition 7.11. Let $\mathbf{C}$ be a finite chain complex of projective $\mathbb{Z} \Gamma_{G}$-modules. If $\tilde{\sigma}\left(S_{H} \mathbf{C}\right)=0$ for all $H \in T \backslash T_{i}$, then there is an $n$ such that $\widetilde{\sigma}\left(S_{H}\left(*_{n} \mathbf{C}\right)\right)=0$ for all $H \in T \backslash T_{i-1}$.

Proof. An element in $\sigma(\mathbf{C})$ can be expressed as a sum $u+\sum_{j} v_{j}+w$ where

$$
u=\sum_{H \in T_{i-1}} \sigma\left(E_{H} S_{H} \mathbf{C}\right), \quad \sum_{j} v_{j}=\sum_{H \in T_{i} \backslash T_{i-1}} \sigma\left(E_{H} S_{H} \mathbf{C}\right),
$$

and

$$
w=\sum_{H \in T \backslash T_{i}} \sigma\left(E_{H} S_{H} \mathbf{C}\right)
$$


By Lemma 7.5, we have

$$
1-\sigma\left(*_{n} \mathbf{C}\right)=(1-\sigma(\mathbf{C}))^{n}=\left(1-\left(u+\sum_{j} v_{j}+w\right)\right)^{n} \in K_{0}\left(\mathbb{Z} \Gamma_{G}\right) .
$$

So,

$$
1-\sigma\left(*_{n} \mathbf{C}\right) \equiv\left(1-\left(\sum_{j} v_{j}+w\right)\right)^{n} \bmod K_{0}\left(\mathbb{Z} \Gamma_{G}\right)_{i-1} .
$$

By Lemma 7.9, it is easy to see that $v_{j} \cdot v_{k} \equiv 0\left(\bmod K_{0}\left(\mathbb{Z} \Gamma_{G}\right)_{i-1}\right)$, for $j \neq k$. Note that

$$
v_{j} \cdot v_{j} \equiv \text { stably free }\left(\bmod K_{0}\left(\mathbb{Z} \Gamma_{G}\right)_{i-1}\right)
$$

by Proposition 7.7. To complete the proof, observe that modulo $K_{0}\left(\mathbb{Z} \Gamma_{G}\right)_{i-1}$,

$$
\begin{aligned}
1-\sigma\left(*_{n} \mathbf{C}\right) & \equiv\left(1-\left(\sum_{j} v_{j}+w\right)\right)^{n} \\
& \equiv 1+\sum_{k=1}^{n}\left(\begin{array}{l}
n \\
k
\end{array}\right)(-1)^{k}\left(\sum_{j} v_{j}+w\right)^{k} \\
& =1+\sum_{k=1}^{n}\left(\begin{array}{l}
n \\
k
\end{array}\right)(-1)^{k} \sum_{j} k v_{j} w^{k-1}+\text { stably free } \\
& =1+n \sum_{k=1}^{n} \sum_{j}\left(\begin{array}{l}
n-1 \\
k-1
\end{array}\right)(-1)^{k} v_{j} w^{k-1}+\text { stably free. }
\end{aligned}
$$

This shows that $\sigma\left(*_{n} \mathbf{C}\right)$ is stably free for some $n$, since $\tilde{K}_{0}\left(\mathbb{Z} \Gamma_{G}\right)$ is a finite group.

\section{Realization of free chain complexes}

Let $X$ be $G$-CW complex, and let $\mathcal{F}$ be a family of subgroups of $G$. Throughout this section, $R$ denotes a commutative ring and $\Gamma_{G}$ denotes the orbit category $\operatorname{Or}_{\mathcal{F}} G$.

Definition 8.1. We say that a $G$-CW complex $X$ has isotropy in $\mathcal{F}$, provided that $X^{H} \neq \emptyset$ implies $H \in \mathcal{F}$, for all $H \leq G$.

The main result of this section is Theorem 8.10, which shows that under certain conditions a finite free chain complex over the orbit category can be realized by a finite $G-\mathrm{CW}$ complex with isotropy in $\mathcal{F}$. This is a generalization of Swan [32], Theorem A, which is based on a construction of Milnor, see 3.1 in [32]. 
Associated to a $G$-CW complex $X$ with isotropy in $\mathcal{F}$, there is a chain complex of $R \Gamma_{G}$-modules defined by

$$
\mathbf{C}\left(X^{?} ; R\right): \quad \cdots \stackrel{\partial_{n+1}}{\longrightarrow} R\left[X_{n}{ }^{?}\right] \stackrel{\partial_{n}}{\longrightarrow} R\left[X_{n-1}{ }^{?}\right] \rightarrow \cdots \stackrel{\partial_{1}}{\longrightarrow} R\left[X_{0}{ }^{?}\right] \rightarrow 0
$$

where $X_{i}$ denotes the set of $i$-dimensional cells in $X$ and $R\left[X_{i}{ }^{?}\right]$ is the coefficient system with $R\left[X_{i}{ }^{?}\right](H)=R\left[X_{i}^{H}\right]$. We denote the homology of this complex by $H_{*}\left(X^{?} ; R\right)$, and in particular

$$
H_{*}\left(X^{?} ; R\right)(H)=H_{*}\left(X^{H} ; R\right) .
$$

Given a chain complex $\mathbf{C}$ of $R \Gamma_{G}$-modules, there is a dimension function

$$
\operatorname{Dim} \mathbf{C}: \mathcal{F} \rightarrow \mathbb{Z}
$$

constant on conjugacy classes of subgroups, defined by

$$
(\operatorname{Dim} \mathbf{C})(H)=\operatorname{dim} \mathbf{C}(H),
$$

for all $H \in \mathcal{F}$, where the dimension of a chain complex of $R$-modules is defined in the usual way as the largest integer $d$ such $C_{d} \neq 0$.

It will be convenient to write $(H) \leq(K)$ whenever $H^{g} \leq K$ for some $g \in G$. Here $(H)$ denotes the set of subgroups conjugate to $H$ in $G$.

Definition 8.2. We call a function $\underline{d}: \mathcal{F} \rightarrow \mathbb{Z}$ monotone if it satisfies the property that $\underline{d}(K) \leqslant \underline{d}(H)$ whenever $(H) \leq(K)$. We say that a monotone function $\underline{d}$ is strictly monotone if $\underline{d}(K)<\underline{d}(H)$, whenever $(H) \leq(K)$ and $(H) \neq(K)$.

Note that $\underline{d}$ monotone implies that $\underline{d}$ is constant on conjugacy classes (such functions are usually called super class functions). We remark that the dimension function of a projective chain complex is always monotone: if $\left(E_{H} P\right)(K) \neq 0$, then $\left(E_{H} P\right)(L) \neq 0$ for every $L \leq K$.

A chain complex $\mathbf{C}$ of $R \Gamma_{G}$-modules is connected if $\mathbf{C}$ is positive and $H_{0}(\mathbf{C})=\underline{R}$.

Definition 8.3. Let $\underline{n}: \mathcal{F} \rightarrow \mathbb{Z}$ be a monotone, non-negative function. A complex $\mathbf{C}$ of $R \Gamma_{G}$-modules is called an $\underline{n}$-Moore complex if it is connected, and for all $H \in \mathcal{F}$, the reduced homology $\widetilde{H}_{i}(\mathbf{C}(H))=0$, for $i \neq \underline{n}(H)$.

A special case of an $\underline{n}$-Moore complex is a homology $\underline{n}$-sphere.

Definition 8.4. We say that a complex $\mathbf{C}$ of $R \Gamma_{G}$-modules is an $R$-homology $\underline{n}$ sphere if it is an $\underline{n}$-Moore complex, and for all $H \in \mathcal{F}$, we have $\widetilde{H}_{i}(\mathbf{C}(H)) \cong \bar{R}$, for $i=\underline{n}(H)$. A homology $\underline{n}$-sphere is called oriented if the $N_{G}(H) / H$-action is trivial on the homology of $\mathbf{C}(H)$ for all $H \in \mathcal{F}$. 
The chain complex associated to the unit sphere $X=S(V)$ of a real or complex representation $V$ of $G$ is an example of a $\mathbb{Z}$-homology $\underline{n}$-sphere, where $\underline{n}(H)=$ $\operatorname{dim} X^{H}$. A $G$-CW complex $X$ with this property is a homotopy representation in the sense of tom Dieck (see [10], Chapter II, Definition 10.1), provided that its dimension function is strictly monotone. We will not use this terminology further.

We now introduce a technique to remove free modules above the homological dimension from a chain complex, without changing its chain homotopy type. For this delicate process we first need some algebraic lemmas.

Definition 8.5. Let $\Gamma$ be an EI-category. A free $R \Gamma$-module $F$ is called isotypic of type $x \in \mathrm{Ob}(\Gamma)$ if it is isomorphic to a direct sum of copies of the free module $E_{x} R[x]$.

For extensions involving isotypic modules we have a splitting property.

Lemma 8.6. Let

$$
\varepsilon: 0 \rightarrow F \rightarrow F^{\prime} \rightarrow M \rightarrow 0
$$

be a short exact sequence of $R \Gamma$-modules over an EI-category $\Gamma$, such that both $F$ and $F^{\prime}$ are isotypic free modules of the same type $x \in \mathrm{Ob}(\Gamma)$. If $M(x)$ is $R$-torsion free, then $\&$ splits and $M$ is stably free.

Proof. It is enough to prove the result in case $F=E_{x} R[x]$, where $x \in \mathrm{Ob} \Gamma$. The general case follows from this by an easy induction. Consider the extension

$$
\varepsilon: 0 \rightarrow E_{x} R[x] \stackrel{j}{\rightarrow} F \rightarrow M \rightarrow 0 .
$$

By the adjointness property

$$
\operatorname{Hom}_{R \Gamma}\left(E_{x} R[x], N\right) \cong \operatorname{Hom}_{R[x]}(R[x], N(x))
$$

for any $R \Gamma$-module $N$. We apply this to the given injection $j: E_{x} R[x] \rightarrow F^{\prime}=$ $\left(E_{x} R[x]\right)^{m}$. Since

$$
\mathcal{E}(x): 0 \rightarrow R[x] \stackrel{j}{\rightarrow} R[x]^{m} \rightarrow M(x) \rightarrow 0
$$

has $R$-torsion free cokernel $M(x)$, this sequence splits over $R[x]$. By the naturality of the adjointness property, we get a splitting of $j$ over $R \Gamma$.

Recall that hdim $\mathbf{C}(H)$ denotes the homological dimension of the chain complex $\mathbf{C}(H)$.

Proposition 8.7. Let $\mathbf{C}$ be a finite free chain complex of $R \Gamma_{G}$-modules, and let $H \in \mathcal{F}$ have the property that $\operatorname{hdim} \mathbf{C}(H)<d:=\operatorname{dim} \mathbf{C}(H)$. Suppose that $\operatorname{dim} \mathbf{C}(K) \leq(d-2)$ for all $(H) \leq(K),(H) \neq(K)$. Then $\mathbf{C} \simeq \mathbf{D}$, where $\mathbf{D}$ is a finite free complex with $\operatorname{dim} \mathbf{D}(H)=d-1$, and $\operatorname{dim} \mathbf{D}(K)=\operatorname{dim} \mathbf{C}(K)$ for all $(K) \neq(H)$. 
Proof. Consider the subcomplex $\mathbf{C}^{\prime}$ of $\mathbf{C}$ formed by free summands of $\mathbf{C}$ isomorphic to $\mathbb{Z}\left[G / K^{?}\right]$, with $(G / K)^{H} \neq 0$ or equivalently $(H) \leq(K)$. The boundary maps of $\mathbf{C}^{\prime}$ are the restrictions of the usual boundary maps to these submodules. Since $\operatorname{dim} \mathbf{C}(K) \leqslant(d-2)$ for all $(H) \leq(K)$ such that $(H) \neq(K)$, the free modules $C_{d}^{\prime}$ and $C_{d-1}^{\prime}$ are isotypic of type $G / H$. We have

$$
\mathbf{C}^{\prime}: 0 \rightarrow C_{d}^{\prime} \rightarrow C_{d-1}^{\prime} \rightarrow \cdots \rightarrow C_{1}^{\prime} \rightarrow C_{0}^{\prime} \rightarrow 0
$$

where $d=\operatorname{dim} \mathbf{C}(H)$. Note that $\mathbf{C}(H)=\mathbf{C}^{\prime}(H)$, so the map $\partial_{d}: C_{d}^{\prime} \rightarrow C_{d-1}^{\prime}$ is injective by the condition that $\operatorname{hdim} \mathbf{C}(H)<\operatorname{dim} \mathbf{C}(H)$. Now we can apply Lemma 8.6 to the extension

$$
0 \rightarrow C_{d}^{\prime} \stackrel{\partial_{d}}{\longrightarrow} C_{d-1}^{\prime} \rightarrow \text { coker } \partial_{d} \rightarrow 0
$$

and conclude that coker $\left(\partial_{d}\right)$ is a stably free $R \Gamma_{G}$-module. By adding elementary chain complexes to $\mathbf{C}$ of the form $\mathbb{Z}\left[G / H^{\text {? }}\right] \stackrel{\text { id }}{\rightarrow} \mathbb{Z}\left[G / H^{?}\right]$ in the adjacent dimensions $(d-1)$ and $(d-2)$, we can assume that coker $\left(\partial_{d}\right)$ is free. Consider the diagram

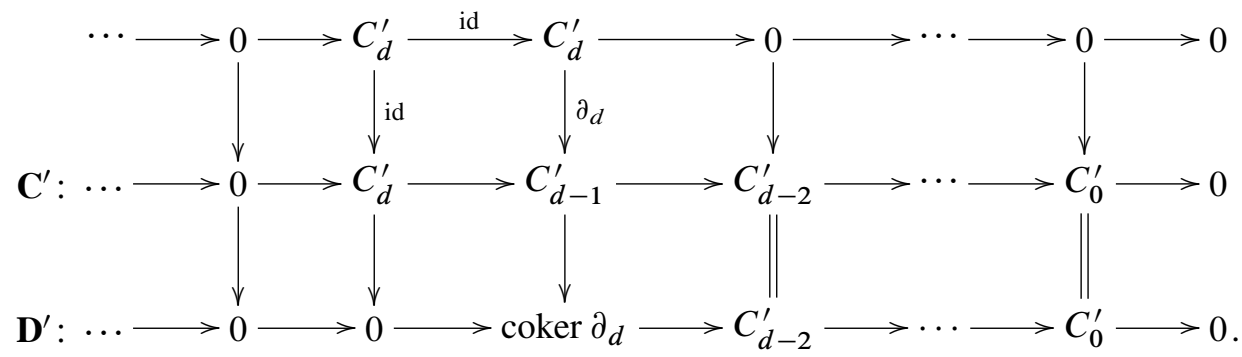

The chain complex $\mathbf{D}^{\prime}$ is a chain complex of free modules and it is chain homotopy equivalent to $\mathbf{C}^{\prime}$. Now define $\mathbf{D}$ as the push-out in the following diagram:

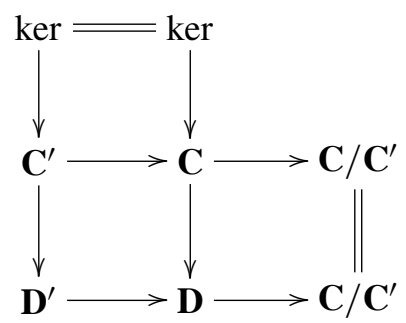

Since, $\mathbf{C}^{\prime}$ and $\mathbf{D}^{\prime}$ are chain homotopy equivalent, then $\mathbf{C}$ and $\mathbf{D}$ are chain homotopy equivalent. Also, note that $\operatorname{dim} \mathbf{D}(H)=\operatorname{dim} \mathbf{D}^{\prime}(H)=(d-1)$, and $\operatorname{dim} \mathbf{D}(K)=$ $\operatorname{dim} \mathbf{C}(K)$ for all $(K) \neq(H)$.

This immediately gives the following. 
Corollary 8.8. Let $\mathbf{C}$ be a finite free chain complex of $R \Gamma_{G}$-modules. Suppose that $\mathbf{C}$ is a homology $\underline{n}$-sphere, with $\underline{n}$ strictly monotone. Then $\mathbf{C}$ is chain homotopy equivalent to a complex $\mathbf{D}$ with $\operatorname{Dim} \mathbf{D}=\underline{n}$.

Proof. Since $\mathbf{C}$ is a homology $\underline{n}$-sphere, $\underline{n}(K)=\operatorname{hdim} \mathbf{C}(K)$, for all $K \in \mathcal{F}$. We apply the previous result to a subgroup $H$, which is maximal with respect to the property that $\operatorname{hdim} \mathbf{C}(H)<d:=\operatorname{dim} \mathbf{C}(H)$. Then $n(K)=\operatorname{dim} \mathbf{C}(K)$ for all $K \in \mathcal{F}$ larger than $H$. Since $\underline{n}$ is strictly monotone, $\operatorname{dim} \mathbf{C}(K) \leqslant(d-2)$ for all $(H) \leq(K),(H) \neq(K)$. This process can be repeated until $\operatorname{Dim} \mathbf{D}=\underline{n}$.

When the dimension function of $\mathbf{C}$ is not strictly monotone, we get a weaker result. Following Section 2, we define $l(H, K)$ as the maximum length of a chain of conjugacy classes of subgroups

$$
(H)=\left(H_{0}\right) \varsubsetneqq\left(H_{1}\right) \varsubsetneqq \ldots \varsubsetneqq\left(H_{l}\right)=(K)
$$

where all $H_{i} \in \mathcal{F}, 0 \leqslant i \leqslant l$.

Corollary 8.9. Let $\mathbf{C}$ be a finite free chain complex of $R \Gamma_{G}$-modules, and let $\underline{n}: \mathcal{F} \rightarrow$ $\mathbb{Z}$ be a monotone function such that $\operatorname{hdim} \mathbf{C}(H) \leqslant \underline{n}(H)$ for all $H \in \mathscr{F}$. Assume that $l(H, K) \leqslant k$ whenever $\underline{n}(H)=\underline{n}(K)$. Then, $\mathbf{C}$ is chain homotopy equivalent to a complex $\mathbf{D}$ which satisfies $D_{i}(H)=0$ for all $i>\underline{n}(H)+k$.

Proof. Let

$$
(H)=\left(H_{0}\right) \lesseqgtr\left(H_{1}\right) \lesseqgtr \ldots \lesseqgtr\left(H_{l}\right)=(K)
$$

be a maximal length chain of subgroups in $\mathcal{F}$ with $\underline{n}(H)=\underline{n}(K)$. Since $\underline{n}$ is monotone, $\underline{n}\left(H_{i}\right)=\underline{n}(H)$ for $0 \leqslant i \leqslant l$. By repeated application of Proposition 8.7, working down from the maximal element $K$, we can obtain $\operatorname{dim} \mathbf{C}\left(H_{l-i}\right)=\underline{n}(H)+i$, for $0 \leqslant i \leqslant l$. Since $l=l(H, K) \leqslant k$, we have $\operatorname{dim} \mathbf{C}(H) \leqslant \underline{n}(H)+k$ as required.

The main purpose of this section is to prove the following theorem:

Theorem 8.10 (Pamuk [27]). Let $\mathbf{C}$ be a finite free chain complex of $\mathbb{Z} \Gamma_{G}$-modules. Suppose $\mathbf{C}$ is an $\underline{n}$-Moore complex such that $\underline{n}(H) \geqslant 3$ for all $H \in \mathcal{F}$. Suppose further that $C_{i}(H)=0$ for all $i>\underline{n}(H)+1$, and all $H \in \mathcal{F}$. Then there is a finite $G-C W$ complex $X$, such that $\mathbf{C}\left(X^{?} ; \mathbb{Z}\right)$ is chain homotopy equivalent to $\mathbf{C}$, as chain complexes of $\mathbb{Z} \Gamma_{G}$-modules.

Note that the resulting complex $X$ will have isotropy in $\mathcal{F}$. We first prove a lemma (compare Theorem 13.19 of [20]). 
Lemma 8.11. Let $X$ be a finite $G-C W$ complex. Suppose that we are given a free $\mathbb{Z} \Gamma_{G}$-module $F$, and a $\mathbb{Z} \Gamma_{G}$-module homomorphism $\varphi: F \rightarrow H_{n}\left(X^{?} ; \mathbb{Z}\right)$, for some $n \geqslant 2$. Assume further that $X^{H}$ is $(n-1)$-connected for every $H \in \mathcal{F}$ such that $\mathbb{Z}\left[G / H^{?}\right]$ is a summand of $F$. Then, by attaching $(n+1)$-cells to $X$, we can obtain a $G-C W$ complex $Y$ such that

$$
H_{i}\left(X^{?} ; \mathbb{Z}\right) \cong H_{i}\left(Y^{?} ; \mathbb{Z}\right) \text { for } i \neq n, n+1,
$$

and

$$
0 \rightarrow H_{n+1}\left(X^{?} ; \mathbb{Z}\right) \rightarrow H_{n+1}\left(Y^{?} ; \mathbb{Z}\right) \rightarrow F \stackrel{\varphi}{\rightarrow} H_{n}\left(X^{?} ; \mathbb{Z}\right) \rightarrow H_{n}\left(Y^{?} ; \mathbb{Z}\right) \rightarrow 0
$$

is exact.

Proof. Let $Z$ be a wedge of $n$-spheres with a $G$ action on them such that $\widetilde{H}_{n}\left(Z^{?} ; \mathbb{Z}\right) \cong$ $F$ as $\mathbb{Z} \Gamma_{G}$-modules. We want to construct a map $f: Z \rightarrow X$ realizing $\varphi$. But $H_{n}\left(X^{H} ; \mathbb{Z}\right) \cong \pi_{n}\left(X^{H}\right)$, for every $H \in \mathcal{F}$ such that $\mathbb{Z}\left[G / H^{?}\right]$ is a summand of $F$, since $X^{H}$ is assumed to be $(n-1)$-connected. Therefore, we can represent the images of an $\mathbb{Z}\left[N_{G}(H) / H\right]$-basis under $\varphi$ for the isotypic summand in $F$ of type $G / H$ by maps $f_{i}: S^{n} \rightarrow X^{H}$. We extend these maps equivariantly to maps $\bar{f}_{i}: S^{n} \times G / H \rightarrow X$. By repeating this construction for each type $G / H$ in $F$, we obtain an equivariant map $f: Z \rightarrow X$ realizing $\varphi$. Take $Y$ to be the mapping cone of $f$. Then, it is easy to see that $Y$ satisfies the desired conditions.

We also need the following lemma:

Lemma 8.12. Let $\mathbf{C}$ be a finite free chain complex of $\mathbb{Z} \Gamma_{G}$-modules. Suppose that $\mathbf{C}$ is connected, and $H_{i}(\mathbf{C})=0$, for $i=1,2$. Then, $\mathbf{C}$ is chain homotopy equivalent to a complex of the form

$$
\cdots \rightarrow C_{n} \rightarrow C_{n-1} \rightarrow \cdots \rightarrow C_{3} \rightarrow C_{2}(X) \rightarrow C_{1}(X) \rightarrow C_{0}(X) \rightarrow 0
$$

where $C_{2}(X) \rightarrow C_{1}(X) \rightarrow C_{0}(X) \rightarrow 0$ is the initial part of the chain complex $\mathbf{C}\left(X^{?} ; \mathbb{Z}\right)$, for some $G-C W$ complex $X$ with isotropy in $\mathcal{F}$, and $X^{H}$ simply-connected for all $H \in \mathscr{F}$.

Proof. There is a $G-\mathrm{CW}$ complex $E_{\mathscr{F}} G$ satisfying the following properties:

(i) All isotropy subgroups of $E_{\mathscr{F}} G$ are in $\mathcal{F}$.

(ii) For every $H \in \mathcal{F}$, the fixed point $\operatorname{set}\left(E_{\mathcal{F}} G\right)^{H}$ is contractible [22], Theorem 1.9.

The chain complex $\mathbf{D}:=\mathbf{C}\left(\left(E_{\mathcal{F}} G\right)^{?} ; \mathbb{Z}\right)$ of this space gives a free resolution of $\underline{\mathbb{Z}}$ as a $\mathbb{Z} \Gamma_{G}$-module. Since $H_{i}(\mathbf{C})=0$, for $i=1,2$, the following sequences are 
both exact

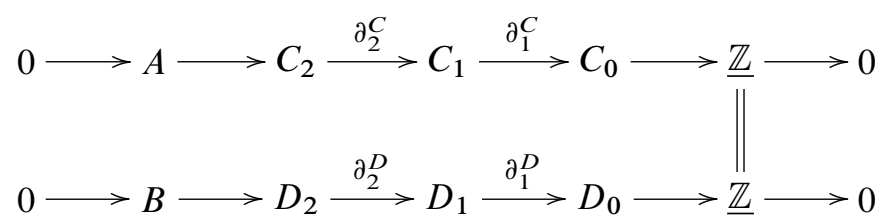

where $A=\operatorname{ker} \partial_{2}^{C}$ and $B=\operatorname{ker} \partial_{2}^{D}$.

By an elementary operation on a sequence $A \rightarrow C_{2} \rightarrow C_{1} \rightarrow C_{0}$ we mean adding or removing trivial free summands $F \stackrel{\text { id }}{\rightarrow} F$ in adjacent dimensions. It is clear that elementary operations do not change the chain homotopy type of the upper and lower sequences in diagram (8.13).

Then, by Schanuel's Lemma [32], 1.1, there exist free modules $F$ and $F^{\prime}$ such that $A \oplus F \cong B \oplus F^{\prime}$. In fact, the argument in Schanuel's lemma can be extended to say that the induced isomorphism $\gamma: A \oplus F \cong B \oplus F^{\prime}$ comes from a chain isomorphism after a sequence of elementary operations (compare [20], p. 279).

In other words, there exists a chain isomorphism

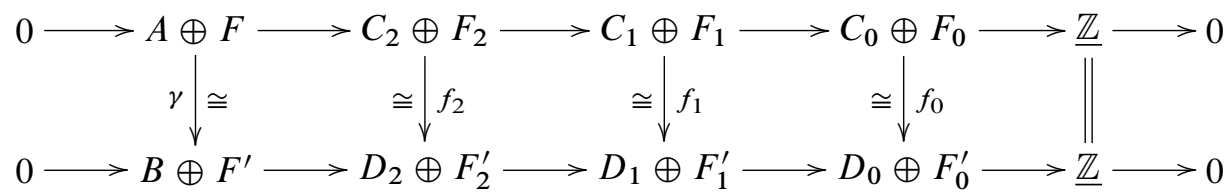

for some suitable choices of free modules, where the upper and lower sequences in diagram (8.14) are obtained from those in diagram (8.13) by elementary operations (see Proposition 3.3.3 in [27]).

In the first step, we stabilize $\left(A \rightarrow C_{2}\right) \mapsto\left(A \oplus F \rightarrow C_{2} \oplus F\right)$, by adding the identity on $F$, and similarly $\left(B \rightarrow D_{2}\right) \mapsto\left(B \oplus F^{\prime} \rightarrow D_{2} \oplus F^{\prime}\right)$. We therefore have a chain map

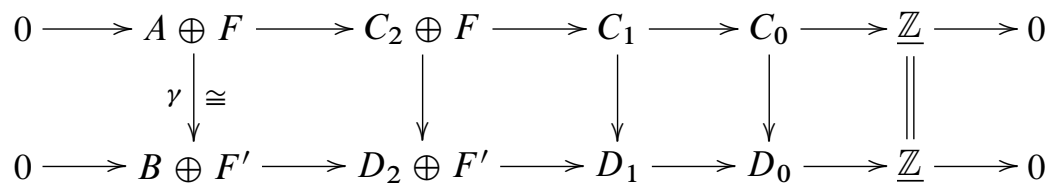

which is a chain homotopy equivalence (by composition with the chain map in (8.14)). After an elementary operation on $\mathbf{C}$, we can use the isomorphism $\gamma: A \oplus F \cong B \oplus F^{\prime}$ to splice the bottom sequence to $\mathbf{C}$, and obtain a chain homotopy equivalence

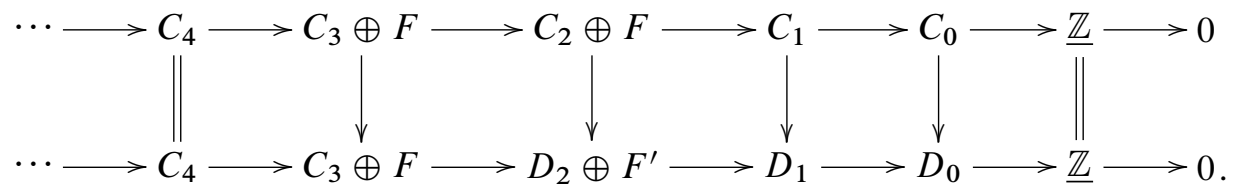


The top sequence is chain homotopy equivalent to $\mathbf{C}$, so to complete the proof we need to show that the sequence $D_{2} \oplus F^{\prime} \rightarrow D_{1} \rightarrow D_{0} \rightarrow 0$ can be realized as the first three terms of a chain complex of a $G$-CW complex $X$, with isotropy in $\mathcal{F}$, such that $X^{H}$ is simply connected for all $H \in \mathscr{F}$ : since $E_{\mathscr{F}} G$ is contractible, using Lemma 8.11, we can attach free 2-cells to its two skeleton $E_{\mathscr{F}} G^{(2)}$. The resulting complex $X$ will have the desired properties.

Now, we are ready to prove Theorem 8.10.

Proof of Theorem 8.10. We can assume that the complex $\mathbf{C}$ is of the form given in Lemma 8.12. We obtain a map $\varphi: C_{3} \rightarrow C_{2}\left(X^{(2)}\right)$ which induces an isomorphism $Z_{2}(\mathbf{C}) \rightarrow Z_{2}\left(X^{(2)}\right)$ between 2-cycles of these chain complexes. This is the starting point for an inductive argument based on applying Lemma 8.11 at each step.

Fix $n \geqslant 2$, and assume by induction that there is an $n$-dimensional $G$-CW complex $X^{(n)}$, and a chain map

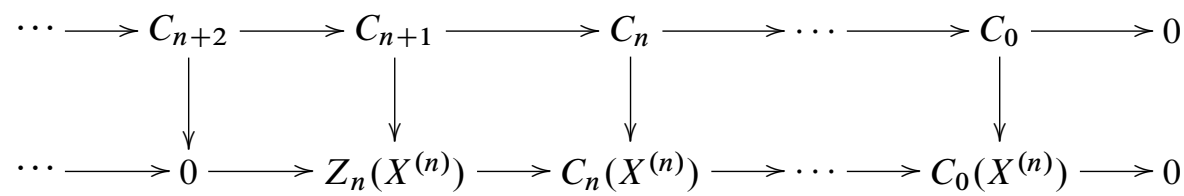

which induces an homology isomorphism for dimensions less than or equal to $(n-1)$, and at dimension $n$ the induced map $Z_{n}(\mathbf{C}) \rightarrow Z_{n}\left(X^{(n)}\right)$ is an isomorphism.

Note that $\operatorname{dim} \mathbf{C}(H) \leqslant \underline{n}(H)+1$ by assumption. If $\mathbb{Z}\left[G / H^{?}\right]$ is a summand of $C_{n+1}$, then $(n+1) \leqslant \operatorname{dim} \mathbf{C}(H) \leqslant \underline{n}(H)+1$ implies $\underline{n}(H) \geqslant n$, and hence the $H$-fixed set of $X^{(n)}$ is $(n-1)$-connected. We can now apply Lemma 8.11 to the map $\varphi: C_{n+1} \rightarrow H_{n}\left(X^{(n)} ; \mathbb{Z}\right)$ defined by the composition

$$
\phi: C_{n+1} \rightarrow Z_{n}(\mathbf{C}) \cong Z_{n}\left(X^{(n)}\right) \rightarrow H_{n}\left(X^{(n)} ; \mathbb{Z}\right) .
$$

Let us call the resulting complex $X^{(n+1)}$. Note that there is a chain map $\mathbf{C} \rightarrow$ $\mathbf{C}\left(X^{(n+1)}\right)$ which induces an isomorphism on homology for dimensions $\leqslant n$, and at dimension $n+1$ we have an isomorphism $Z_{n+1}(\mathbf{C}) \rightarrow Z_{n+1}\left(X^{(n+1)}\right)$. Since $\mathbf{C}$ is finite dimensional, after finitely many steps, we will obtain a finite dimensional $G$-CW complex $X$ and a chain map $f: \mathbf{C} \rightarrow \mathbf{C}(X)$ which induces isomorphism on homology for all dimensions. Since both $\mathbf{C}$ and $\mathbf{C}(X)$ are free $\mathbb{Z} \Gamma_{G}$-chain complexes, $f$ is a chain homotopy equivalence as desired.

\section{The proof of Theorem A}

Let $G=S_{5}$, the symmetric group of order 120 permuting $\{1,2,3,4,5\}$, and let $S_{4} \leq G$ denote the permutations fixing $\{5\}$. We work relative to the family $\mathscr{F}$ of 
rank 1 subgroups of 2-power order. Let $\Gamma_{G}=\operatorname{Or}_{\mathcal{F}} G$. Our family $\mathcal{F}$ consists of the subgroups of $G$ which are conjugate to one the subgroups in the set

$$
\left\{1, C_{2}^{A}, C_{2}^{B}, C_{4}\right\}
$$

where $C_{2}^{A}=\langle(12)(34)\rangle, C_{2}^{B}=\langle(12)\rangle$, and $C_{4}=\langle(1234)\rangle$. In addition we will consider the Sylow subgroups $C_{3}=\langle(123)\rangle$ and $C_{5}=\langle(12345)\rangle$. It is convenient to note that for $H=S_{4} \leq G$, we have

$$
N_{H}\left(C_{4}\right)=D_{8}=N_{H}\left(C_{2}^{A}\right)=N_{G}\left(C_{4}\right),
$$

while $N_{H}\left(C_{2}^{B}\right)=E=\langle(12),(34)\rangle$, and $N_{H}\left(C_{3}\right)=S_{\{123\}}$. On the other hand, $N_{G}\left(C_{2}^{B}\right)=\left\langle(12), S_{\{345\}}\right\rangle$ and $N_{G}\left(C_{3}\right)=S_{\{123\}} \times\langle(45)\rangle$.

Our strategy will be to construct finite projective complexes $\mathbf{C}^{(p)}$ with isotropy in $\mathscr{F}$ over $\mathbb{Z}_{(p)} \Gamma_{G}$, for each prime $p$ dividing the order of $|G|$, which are $R$-homology $\underline{n}$-spheres with respect to the the same homology dimension function $\underline{n}$. The gluing theory of Section 6, Theorem 6.7, will be used to construct a finite projective $\mathbb{Z}$-homology $\underline{n}$-sphere over $\mathbb{Z} \Gamma_{G}$ from this data. Then the join construction from Section 7 will allow us to find a finite free complex, to which the realization theorem of Section 8 will apply.

We introduce the notation $\underline{R}_{0}$ for the $R \Gamma_{G}$-module defined by $\underline{R}_{0}(K)=0$, for $K \neq 1$, and $\underline{R}_{0}(1)=R$ with trivial $G$-action. In other words, $\underline{R}_{0}=I_{1}(R)$ as defined in Section 2.

9A. The case $\boldsymbol{p}=2$. Let $H=S_{4} \leq G, R=\mathbb{Z}_{(2)}$ and consider the standard $H$-action on the 2-sphere given by the rotational symmetries of the octahedron. Let $X$ denote the $H$-CW complex associated to the first barycentric subdivision of the octahedron. Then $X$ has isotropy in the family consisting of the cyclic subgroups of $H$ of orders $\leq 4$.

Let $\Gamma_{H}=\mathrm{Or}_{\mathscr{F}} H$ denote the orbit category for $H$ with respect to the family $\mathscr{F}_{H}=\mathscr{F}_{H} \cap H$. Consider the chain complex $\mathbf{C}\left(X^{?} ; R\right)$ as a chain complex of $R \Gamma_{H^{-}}$ modules, by restricting this functor to the full subcategory $\Gamma_{H}$ of the orbit category $\operatorname{Or}(H)$. This gives an exact sequence of the form

$$
\begin{aligned}
0 \rightarrow \underline{R}_{0} & \rightarrow 2 R\left[H / 1^{?}\right] \rightarrow 3 R\left[H / 1^{?}\right] \\
& \rightarrow R\left[H / C_{4}{ }^{?}\right] \oplus R\left[H / C_{2}^{B}{ }^{?}\right] \oplus R\left[H / C_{3}{ }^{?}\right] \rightarrow \mathbf{H}_{0} \rightarrow 0
\end{aligned}
$$

where all the modules in the extension (excluding the ends) except $R\left[H / C_{3}{ }^{?}\right]$ are free $R \Gamma_{H}$-modules, and $\mathbf{H}_{0}=H_{0}\left(\mathbf{C}\left(X^{?} ; R\right)\right)$.

Since $R\left[H / C_{3}\right]$ is a projective $R H$-module (it is induced up from $R$, which is projective over $R\left[C_{3}\right]$ ), we see that $R\left[H / C_{3}{ }^{?}\right]=I_{1} R\left[H / C_{3}\right]$ as $R \Gamma_{H}$-modules. Therefore $\mathbf{C}\left(X^{?} ; R\right)$ is a finite projective chain complex over $R \Gamma_{H}$. 
It is useful to represent an $R \Gamma_{H}$-module $M$ by a labelled tree diagram:

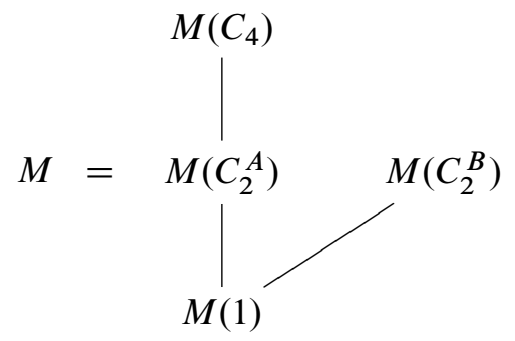

with one vertex for each isomorphism class of objects, and edges given by the partial ordering of the subgroups in $\mathscr{F}$ up to conjugacy. The labels are given by the $R\left[N_{H}(K) / K\right]$-modules $M(K)$, for $K \in \mathcal{F}$.

For the homology module $H_{0}\left(X^{?} ; R\right)$ over the orbit category $\Gamma_{H}$ we have the diagram

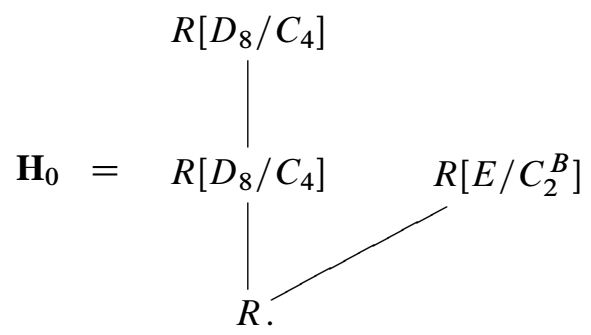

The $(k+1)$-fold join of $\mathbf{C}\left(X^{?} ; R\right)$ with itself (see Section 7) is a finite projective complex of the form

$$
\mathrm{C}: 0 \rightarrow \underline{R}_{0} \rightarrow C_{n} \rightarrow \cdots \rightarrow C_{k} \rightarrow \cdots \rightarrow C_{0} \rightarrow \underline{R} \rightarrow 0
$$

over $R \Gamma_{H}$ with $(n+1)=3(k+1)$. In case $X=S(V)$, where $V \cong R^{3}$ is a real orthogonal $H$-representation, then the join construction on spheres just produces the unit sphere $S(V \oplus \cdots \oplus V)$ in the direct sum of $(k+1)$ copies of $V$. This sphere has real dimension $n=3(k+1)-1$. The purpose of the join construction is to produce a complex with dimension gaps between the non-zero homology groups, as required by Theorem 6.7 for gluing the different primes together.

We have $H_{0}(\mathbf{C})=\underline{R}$ and $H_{n}=\underline{R}_{0}$. If $(k+1)$ is even, then $H_{k}(\mathbf{C}(Q))=R$, with trivial $N_{H}(Q) / Q$-action, and $\widetilde{H}_{i}(\mathbf{C}(Q))=0$, for $i \neq k$, for each non-trivial $Q \in \mathcal{F}$. By Proposition 6.4, we obtain a chain complex $\mathbf{C}^{(2)}$ of projective $R \Gamma_{G^{-}}$ modules, having homology isomorphic to $R$, with trivial $N_{G}(Q) / Q$-action. By construction, the homology dimension function $\underline{n}$ for $\mathbf{C}^{(2)}$ is the same as for $\mathbf{C}$. Notice that $\underline{n}$ is monotone, but not strictly monotone.

9B. The case $\boldsymbol{p}=3$. Let $R=\mathbb{Z}_{(3)}$ and $K=C_{2}^{B}$. The 3-period of $G=S_{5}$ is four (see [7], Chapter XII, Example 11), so by Swan [32] there exists a periodic projective 
resolution $\mathbf{P}$ with

$$
0 \rightarrow R \rightarrow P_{n} \rightarrow \cdots \rightarrow P_{1} \rightarrow P_{0} \rightarrow R \rightarrow 0
$$

over the group ring $R G$, for any $n$ such that $4 \mid(n+1)$. We will assume that $12 \mid(n+1)$, and let $k$ be defined by the equation $(n+1)=3(k+1)$. Similarly, since $N_{G}(K) / K \cong S_{3}$ also has 3-period 4, we have a chain complex D yielding a periodic projective resolution

$$
0 \rightarrow R \rightarrow D_{k} \rightarrow \cdots \rightarrow D_{0} \rightarrow R \rightarrow 0
$$

over $R S_{3}$. In the rest of this section we let $W_{K}=N_{G}(K) / K$ to simplify the notation.

We want a finite projective chain complex $\mathbf{C}$ over $R \Gamma_{G}$ which fits into an extension of chain complexes

$$
0 \rightarrow E_{1} \mathbf{P} \rightarrow \mathbf{C} \rightarrow I_{K} \mathbf{D} \rightarrow 0
$$

where the induced exact sequence on the 0-th homology

$$
0 \rightarrow \underline{R}_{0} \rightarrow H_{0}(\mathbf{C}) \rightarrow I_{K} R \rightarrow 0
$$

is the non-trivial extension of tree diagrams (with vertices at $\{1, K\}$ )<smiles>[R]C(O)C([R])C([R])(C)C([R])O</smiles>

For a projective $R\left[W_{K}\right]$-module $D$, the module $I_{K} D$ has a finite projective resolution of the form

$$
0 \longrightarrow E_{1} \operatorname{Res}_{1} E_{K} D \longrightarrow E_{K} D \longrightarrow I_{K} D \longrightarrow 0 \text {. }
$$

By definition of the functors $E_{x}$ and $I_{x}$ (see Section 2), the canonical map

$$
E_{x} M \rightarrow I_{x} M \rightarrow 0
$$

is always surjective for any $R[x]$-module $M$. We have $E_{K} R\left[W_{K}\right]=R\left[G / K^{?}\right]$ and hence $E_{K} D$ is projective. Also $\operatorname{Res}_{1} E_{K} D$ is projective, because it is a summand of $R[G / K]$ which is projective as a $\mathbb{Z}_{(3)} G$-module. This shows that, once constructed, $\mathbf{C}$ will be homotopy equivalent to a finite projective chain complex by Proposition 6.2.

Associated to every $R G$-chain map $f: \operatorname{Res}_{1} E_{K} \mathbf{D} \rightarrow \mathbf{P}$, there is a chain complex $\mathrm{C}$ which fits into the push-out diagram

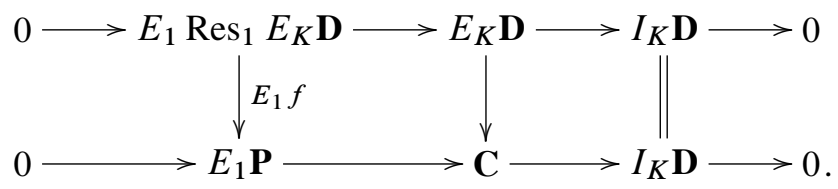


We want to choose $f$ so that $\mathbf{C}$ satisfies the condition on homology. Note that

$$
H_{0}\left(\operatorname{Res}_{1} E_{K} \mathbf{D}\right)=\operatorname{Res}_{1} E_{K} R=R\left[G / N_{G}(K)\right] .
$$

Since the modules $\operatorname{Res}_{1} E_{K} D_{i}=\left(E_{K} D_{i}\right)(1)$ are projective for all $i$ and $\mathbf{P}$ is exact, there exists a chain map $f: \operatorname{Res}_{1} E_{K} \mathbf{D} \rightarrow \mathbf{P}$

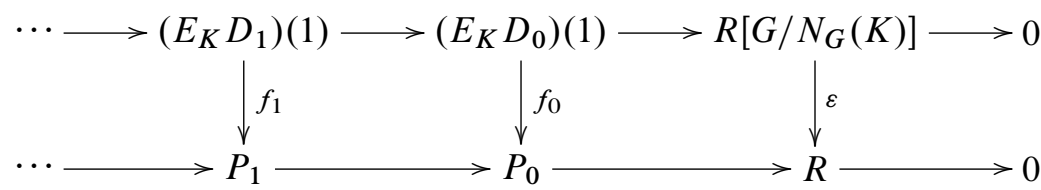

lifting the augmentation map $R\left[G / N_{G}(K)\right] \stackrel{\varepsilon}{\rightarrow} R$. To see that the resulting pushout complex $\mathbf{C}$ has the desired properties, consider the homology at zero for the diagram of chain complexes given above. Since $I_{K}$ is an exact functor, $H_{1}\left(I_{K} \mathbf{D}\right)=$ $I_{K} H_{1}(\mathbf{D})=0$, and we get

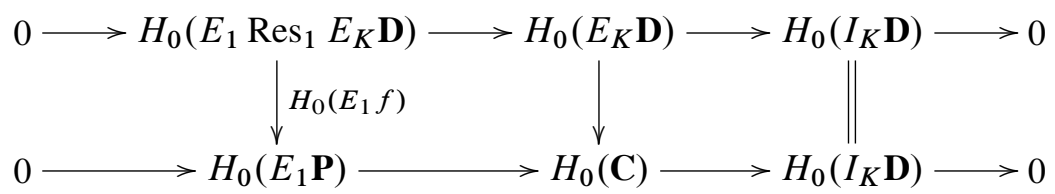

where $H_{0}\left(E_{1} \mathbf{P}\right)=E_{1} R$. Note that

$$
H_{0}\left(E_{1} \operatorname{Res}_{1} E_{K} \mathbf{D}\right)=E_{1} \operatorname{Res}_{1} H_{0}\left(E_{K} \mathbf{D}\right)=E_{1} \operatorname{Res}_{1} E_{K} R=E_{1} R\left[G / N_{G}(K)\right] .
$$

This gives a diagram of the form

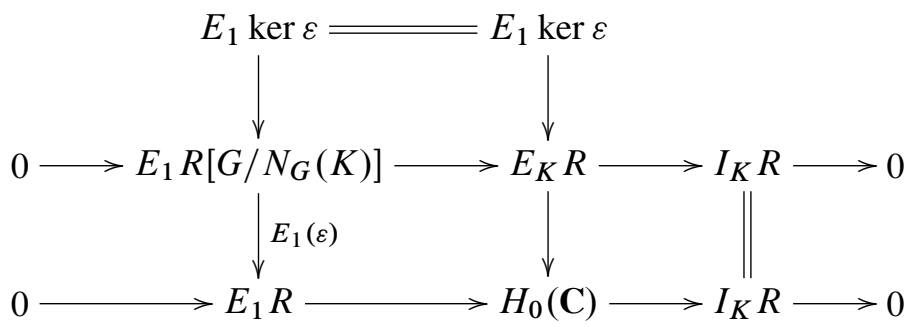

where the middle vertical sequence of $R \Gamma_{G}$-modules is given by

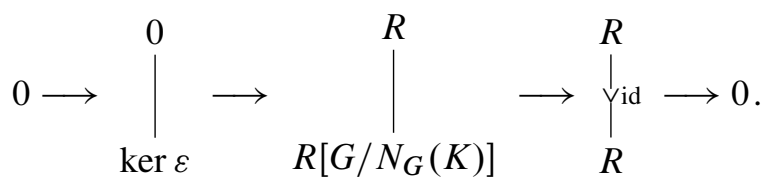

This shows that $H_{0}(\mathbf{C})$ has the desired form. 
Now, to obtain the same homology dimension function as for the complex $\mathbf{C}^{(2)}$, more homology must be added to the complex $\mathbf{C}$. We need to extend $\mathbf{H}_{k}$ and $\mathbf{H}_{0}$ via the non-split extensions

$$
0 \rightarrow \mathbf{H}_{0} \rightarrow \hat{\mathbf{H}}_{0} \rightarrow N \rightarrow 0 \quad \text { and } \quad 0 \rightarrow \mathbf{H}_{k} \rightarrow \hat{\mathbf{H}}_{k} \rightarrow N \rightarrow 0
$$

where

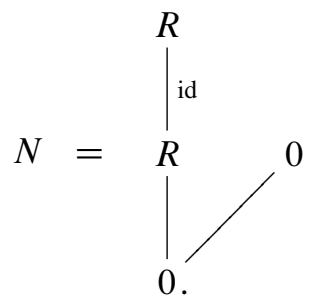

The module $N$ has a finite projective resolution of the form

$$
0 \rightarrow E_{1} R\left[G / D_{8}\right] \rightarrow E_{C_{4}} R \rightarrow N \rightarrow 0 .
$$

Note that $\operatorname{Res}_{1} E_{C_{4}} R=R\left[G / N_{G}\left(C_{4}\right)\right]=R\left[G / D_{8}\right]$, and for $Q=C_{2}^{A}$ we have

$$
\operatorname{Res}_{Q} E_{C_{4}} R=R \otimes_{R\left[D_{8} / C_{4}\right]} R\left[\left(G / C_{4}\right)^{Q}\right]=R \otimes_{R\left[D_{8} / C_{4}\right]} R\left[N_{G}(Q) / N_{C_{4}}(Q)\right]=R
$$

where the equality in the middle comes from Lemma 3.6. Since $R$ is projective as an $R\left[D_{8} / C_{4}\right]$-module, $E_{C_{4}} R$ is projective. It is easy to see that $E_{1} R\left[G / D_{8}\right]$ is also projective. So, by Proposition 6.8, we can replace $\mathbf{C}$ with a finite projective chain complex $\mathbf{C}^{(3)}$ over $R \Gamma_{G}$ which has the desired homology.

9C. The case $\boldsymbol{p}=5$ 5. For $p=5$, the situation is easier than the case $p=3$. Let $R=\mathbb{Z}_{(5)}$. The 5-period of $S_{5}$ equals 8, so by Swan [32] there exists a periodic projective resolution $\mathbf{P}$ over the group ring $R G$, giving an exact sequence

$$
0 \rightarrow R \rightarrow P_{n} \rightarrow \cdots \rightarrow P_{1} \rightarrow P_{0} \rightarrow R \rightarrow 0
$$

for any positive integer $n$ such that $n+1=3(k+1)$ for some integer $k$, with $8 \mid(k+1)$. We start with the $R \Gamma_{G}$-complex $\mathbf{C}=E_{1} \mathbf{P}$ obtained by the extension functor from $\mathbf{P}$. Since $\mathbf{C}$ has no homology at the non-trivial 2-subgroups in $\mathscr{F}$, we need to change the homology at $\mathbf{H}_{0}$ and at $\mathbf{H}_{k}$ to match the homology we have for $p=2$ and $p=3$. Note that we need to extend $\mathbf{H}_{k}$ and $\mathbf{H}_{0}$ via the non-split extensions

$$
0 \rightarrow \mathbf{H}_{0} \rightarrow \hat{\mathbf{H}}_{0} \rightarrow M \rightarrow 0 \text { and } 0 \rightarrow \mathbf{H}_{k} \rightarrow \hat{\mathbf{H}}_{k} \rightarrow M \rightarrow 0
$$


where

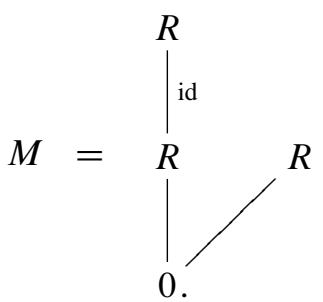

Let $K=C_{2}^{B}$. The module $M$ is the direct sum of $L$ (which has the same form as $N$ ) and $I_{K} R$. We claim that each of these modules have finite projective resolutions over $R \Gamma_{G}$. For $I_{K} R$ we have a resolution of the form

$$
0 \rightarrow E_{1} R\left[G /\left(K \times S_{3}\right)\right] \rightarrow E_{K} R \rightarrow I_{K} R \rightarrow 0 .
$$

Note that

$$
\operatorname{Res}_{1} E_{K} R=R\left[G / N_{G}(K)\right]=R\left[G /\left(K \times S_{3}\right)\right]
$$

where $S_{3}$ denotes the subgroup of $S_{5}$ generated by symmetries of $\{3,4,5\}$. Since $R$ is projective as an $R\left[N_{G}(K) / K\right]$-module, $E_{K} R$ is projective. It is clear that $E_{1} R\left[G /\left(K \times S_{3}\right)\right]$ is also projective. So, the above resolution is a projective resolution of $I_{K} R$. We can also write a finite projective resolution for $L$ (similar to the resolution given for $N$ ). So, by Proposition 6.8, we can replace $\mathbf{C}$ with a finite projective chain complex $\mathbf{C}^{(5)}$ which has the desired homology.

The proof of Theorem $A$. We will first construct a projective chain complex $\mathbf{C}$ over $\mathbb{Z} \Gamma_{G}$ with isotropy in $\mathcal{F}$, by applying Theorem 6.7 to glue the $p$-local complexes $\mathbf{C}^{(p)}$, for $p=2,3,5$. Note that in the constructions of $\mathbf{C}^{(p)}$ above, we may choose any integer $k$ such that $k$ odd, $n+1=3(k+1), 12 \mid(n+1)$ and $8 \mid(k+1)$. To satisfy the first condition in Theorem 6.7, that the distance between non-zero homology groups of the $\mathbf{C}^{(p)}$ is larger than $l\left(\Gamma_{G}\right)=2$, we will also need $k \geq 3$ and $n-k \geq 3$.

Remark 9.1. The minimum value for $k$ satisfying the requirements used above is $k=7$, which gives $n=\operatorname{dim} \mathbf{C}=23$.

The $\mathbb{Z} \Gamma_{G}$-module $\mathbf{H}$ needed to satisfy the second condition in Theorem 6.7 is given by $\mathbf{H}_{i}(K)=\mathbb{Z}$, for $i=0, \underline{n}(K)$ with $K \in \mathcal{F}$, and zero otherwise. By Proposition 6.2, $\mathbf{C}$ is chain homotopy equivalent to a finite projective complex. To obtain a finite free complex, we can apply Theorem 7.6, which (possibly after some joins) produces a finite free $\mathbb{Z} \Gamma_{G}$-chain complex $\mathbf{C}$ with the $\mathbb{Z}$-homology of an $\underline{n}$-sphere, and $\underline{n}(K) \geqslant 3$ for all $K \in \mathcal{F}$.

Note that our homology dimension function $\underline{n}$ is not strictly monotone, since $\underline{n}\left(C_{2}^{A}\right)=\underline{n}\left(C_{4}\right)$, but by Corollary 8.9 we can modify our complex to satisfy the 
conditions for geometric realization in Theorem 8.10, since $l\left(C_{2}^{A}, C_{4}\right)=1$. Applying Theorem 8.10, we conclude that $G=S_{5}$ acts on a finite $G$-CW complex $X$ with isotropy in $\mathcal{F}$.

Remark 9.2. For this particular example we needed to apply Theorem 7.6 with one join tensor operation, because $\widetilde{K}_{0}\left(\mathbb{Z} \Gamma_{G}\right)=\mathbb{Z} / 2$. This follows from Theorem 7.8 , Lemma 7.5 and well-known calculations showing that $\widetilde{K}_{0}\left(\mathbb{Z}\left[N_{G}(Q) / Q\right]\right)=0$, for $1 \neq Q \in \mathcal{F}$, but $\widetilde{K}_{0}(\mathbb{Z}[G])=\mathbb{Z} / 2$. Note that, by Dress induction, it is enough to consider the projective class groups of $p$-hyperelementary subgroups of $G$ (see $\$ 50$ in [9], [28]). We therefore obtain a finite $G$-CW complex $X \simeq S^{47}$ with isotropy in $\mathcal{F}$.

\section{References}

[1] A. Adem and J. H. Smith, Periodic complexes and group actions. Ann. of Math. (2) 154 (2001), 407-435. Zbl 0992.55011 MR 1865976

[2] D. J. Benson, Representations and cohomology. I. Basic representation theory of finite groups and associative algebras.. Second ed., Cambridge Stud. Adv. Math. 30, Cambridge University Press, Cambridge 1998. Zbl 0908.20001 MR 1644252

[3] D. J. Benson, Representations and cohomology. II. Cohomology of groups and modules. Second ed., Cambridge Stud. Adv. Math.31, Cambridge University Press, Cambridge 1998, Zbl 0908.20002 MR 1634407

[4] S. Bouc, Résolutions de foncteurs de Mackey. In Group representations: cohomology, group actions and topology (Seattle, WA, 1996), Proc. Sympos. Pure Math. 63, Amer. Math. Soc., Providence, RI, 1998, 31-83. Zbl 0897.19001 MR 1603131

[5] N. Brady, I. J. Leary and B. E. A. Nucinkis, On algebraic and geometric dimensions for groups with torsion. J. London Math. Soc. (2) 64 (2001), 489-500. Zbl 1016.20035 MR 1853466

[6] G. E. Bredon, Equivariant cohomology theories. Lecture Notes in Math. 34, SpringerVerlag, Berlin 1967. Zbl 0162.27202 MR 0214062

[7] H. Cartan and S. Eilenberg, Homological algebra. With an appendix by David A. Buchsbaum, reprint of the 1956 original, Princeton Landmarks Math., Princeton University Press, Princeton, NJ, 1999. Zbl 0075.24305 MR 1731415

[8] O. Coşkun and E. Yalçın, A Tate cohomology sequence for generalized Burnside rings. $J$. Pure Appl. Algebra 213 (2009), 1306-1315. Zbl 1169.19001 MR 2497578

[9] C. W. Curtis and I. Reiner, Methods of representation theory. Vol. II, John Wiley \& Sons Inc., New York 1987. Zbl 0616.20001 MR 0892316

[10] T. tom Dieck, Transformation groups. De Gruyter Stud. Math. 8, Walter de Gryter, Berlin 1987. Zbl 0611.57002 MR 0889050

[11] A. Dold, Zur Homotopietheorie der Kettenkomplexe. Math. Ann. 140 (1960), 278-298. Zbl 0093.36903 MR 0112906 
[12] R. M. Dotzel and G. C. Hamrick, p-group actions on homology spheres. Invent. Math. 62 (1981), 437-442. Zbl 0453.57030 MR 0604837

[13] A. W. M. Dress, Induction and structure theorems for orthogonal representations of finite groups. Ann. of Math. (2) 102 (1975), 291-325. Zbl 0315.20007 MR 0387392

[14] J. Grodal, Higher limits via subgroup complexes. Ann. of Math. (2) 155 (2002), 405-457. Zbl 1004.55008 MR 1906592

[15] J. Grodal and J. H. Smith, Algebraic models for finite $G$-spaces. Abstracts from the homotopy theory workshop, September 16-22, 2007, Oberwolfach Reports 4, Eur. Math. Soc., Zürich 2007, 2690-2692.

[16] I. Hambleton, L. R. Taylor and E. B. Williams, Dress induction and the Burnside quotient Green ring. Algebra \& Number Theory 3 (2009), 511-541. Zbl 05652980 MR 2578887

[17] S. Jackowski, J. McClure and B. Oliver, Homotopy classification of self-maps of $B G$ via $G$-actions. II. Ann. of Math. (2) 135 (1992), 227-270. Zbl 0771.55003 MR 1154593

[18] M. A. Jackson, Qd ( $p)$-free rank two finite groups act freely on a homotopy product of two spheres. J. Pure Appl. Algebra 208 (2007), 821-831. Zbl 1109.57021 MR 2283428

[19] T.-Y. Lam, Induction theorems for Grothendieck groups and Whitehead groups of finite groups. Ann. Sci. École Norm. Sup. (4) 1 (1968), 91-148. Zbl 0164.02703 MR 0231890

[20] W. Lück, Transformation groups and algebraic K-theory. Lecture Notes in Math. 1408, Springer-Verlag, Berlin 1989. Zbl 0679.57022 MR 1027600

[21] W. Lück, Chern characters for proper equivariant homology theories and applications to $K$ and L-theory. J. Reine Angew. Math. 543 (2002), 193-234. Zbl 0987.55008 MR 1887884

[22] W. Lück, Survey on classifying spaces for families of subgroups. In Infinite groups: geometric, combinatorial and dynamical aspects, Progr. Math. 248, Birkhäuser, Basel 2005, 269-322. Zbl 1117.55013 MR R2195456

[23] S. Mac Lane, Homology. Reprint of the 1975 edition, Classics Math., Springer-Verlag, Berlin 1995. Zbl 0328.18009 MR 1344215

[24] S. Mac Lane, Categories for the working mathematician. Second ed., Grad. Texts in Math. 5, Springer-Verlag, New York 1998. Zbl 0906.18001 MR 1712872

[25] G. Mislin, On group homomorphisms inducing mod- $p$ cohomology isomorphisms. Comment. Math. Helv. 65 (1990), 454-461. Zbl 0713.55009 MR 1069820

[26] B. Oliver and Y. Segev, Fixed point free actions on Z-acyclic 2-complexes. Acta Math. 189 (2002), 203-285. Zbl 1034.57033 MR 1961198

[27] S. Pamuk, Periodic resolutions and finite group actions. Ph.D. thesis, McMaster University, 2008.

[28] I. Reiner, Projective class groups of symmetric and alternating groups. Collection of articles dedicated to Olga Taussky-Todd, Linear and Multilinear Algebra 3 (1975/76), 147-153. Zbl 0327.20003 MR 0390034

[29] D. S. Rim, Modules over finite groups. Ann. of Math. (2) 69 (1959), 700-712. Zbl 0092.26104 MR 0104721

[30] E. H. Spanier, Algebraic topology. McGraw-Hill Book Co., New York 1966. Zbl 0145.43303 MR 0210112

[31] R. G. Swan, Induced representations and projective modules. Ann. of Math. (2) 71 (1960), 552-578. Zbl 0104.25102 MR 0138688 
Vol. 88 (2013)

[32] R. G. Swan, Periodic resolutions for finite groups. Ann. of Math. (2) 72 (1960), 267-291. Zbl 0096.01701 MR 0124895

[33] P. Symonds, Mackey functors and control of fusion. Bull. London Math. Soc. 36 (2004), 623-632. Zbl 1081.20062 MR 2268362

[34] P. Symonds, Smith theory for algebraic varieties. Algebr. Geom. Topol. 4 (2004), 121-131. Zbl 1069.57020 MR 2059185

[35] P. Symonds, The Bredon cohomology of subgroup complexes. J. Pure Appl. Algebra 199 (2005), 261-298. Zbl 1067.18013 MR 2134305

Received July 30, 2008; revised March 30, 2010

Ian Hambleton, Department of Mathematics, McMaster University, Hamilton, Ontario L8S $4 \mathrm{~K} 1$, Canada

E-mail: hambleton@mcmaster.ca

Semra Pamuk, Department of Mathematics, Middle East Technical University, 06531

Ankara, Turkey

E-mail: pasemra@metu.edu.tr

Ergün Yalçın, Department of Mathematics, Bilkent University, 06800 Bilkent, Ankara, Turkey

E-mail: yalcine@fen.bilkent.edu.tr 